Universidad Politécnica de Madrid

Escuela Técnica Superior

POLITÉCNICA

"Ingeniamos el futuro" de Ingenieros Informáticos



PhD. Thesis

\title{
Big Medical Text Analytics: Querying, Searching and Understanding Clinical Data
}

Author: Roberto Costumero Moreno Ingeniero en Informática - MSc Computer Science

Supervisors: Prof. Dr. Ernestina Menasalvas Ruiz

Prof. Dr. Consuelo Gonzalo Martín

Madrid, June, 2017 

Chairwoman: María Amparo Vila Miranda

Universidad de Granada

Member: $\quad$ Sebastián Ventura Soto

Universidad de Córdoba

Member: $\quad$ Pedro Alexandre Sousa

Universidade Nova de Lisboa

Member: José Vivancos Mora

Hospital Universitario de La Princesa

Secretary: Víctor Robles Forcada

Universidad Politécnica de Madrid

Substitute: Alejandro Rodríguez González

Universidad Politécnica de Madrid

Substitute: Juan Miguel Gómez Berbís

Universidad Carlos III de Madrid 

Being at the highest status of Academia, I can only think of my parents that have given everything and fought for me to deliver the best in me.

Rocio, my soulmate, my one. You have always been by my side on the constants ups \& downs that during this stage we have suffered together.

I cannot forget of you, the ones that passed away, specially my grandparents, just because my achievements are only possible going back to the roots. 

The best way to predict the future is to create it.

Abraham Lincoln

Your time is limited; don't waste it living someone else's life.

Steve Jobs 



\section{Acknowledgements}

Four years have passed since I last wrote the acknowledgements in my final master thesis. Many things have changed, others have not. I still think that one should be grateful for what it is deserved. I will write part of these acknowledgements in Spanish for those not so fluent in the language of Shakespeare.

I would like to thank every person that in any way have contributed to the development of this thesis, both professionally and personally in endless interchanges of ideas and both good and not so good moments.

First, I would like to thank my two supervisors, Ernestina Menasalvas and Consuelo Gonzalo, for all the support and all the contributions made to this thesis. You have encouraged me to work in many different projects and to continuously study new things to achieve new goals and to become a more literate person. Thanks for giving me this opportunity and believe in the work we have done in this thesis.

I would also like to thank Victor Robles for being that lighthouse for me in the University, guiding me through the different steps we have shared in the last few years.

I cannot forget about the people in IBM. Looking backwards doing the internship in Ireland was a wonderful experience, and I have to thank specially DJ McCloskey and Anthony Kelly for treating me like anyone else in the team and teaching me so many valuable things. Of course I have to thank all my colleagues: Ronan, Mikhail, Urvesh, Mohamed, Alexey, Ross, Ahmed, Kay, Keith... Of course, I would like also to give special thanks to Elisa Martín Garijo, Victor Camargo, Alejandro Delgado and Soledad Liniers for the support and giving me the opportunity to do that internship and all the work we have done with Watson at UPM. I would also like to thank Armen Pischdotchian for sharing wonderful experiences in Dublin and Madrid.

To all the people in EIT Digital that have helped and taught me a lot in the last few years about the industry and helped me to grow both professionally and personally. Special thanks to Susana Eiroa and Susana Negrete, for helping in so many different tasks related to EIT Digital and to be there to help me whenever I needed. Thanks to Gonzalo León, Martin Vendel, Alessandro Rossi, and Jussi Autere for organizing such inspiring courses. EIT Digital, as an institution, has expanded my vision and enlightened with new ideas in this process, and given me funding and opportunities to develop the industrial part of this thesis.

I would like also to thank the people in ActúaUPM for all the work they do in developing new ventures inside the university and for sharing so many experiences, in particular: Arístides, Iván, Alberto, Elena, Beatriz and of course Elisa, for helping in the development of $\mathrm{H} 2 \mathrm{~A}$ business plan and being there to listen and chat so many times. 
0\%... Switching language... $100 \%$ complete.

Me gustaría terminar los agradecimientos "académicos" dando las gracias a los profesores de mi grupo docente: Covadonga, Ernestina, Luis y Alejandro por acogerme en el grupo y demostrar tanta confianza en mi trabajo docente. Mención especial los profesores que a lo largo de la carrera y del doctorado me han formado como persona: Ricardo Imbert, Marisa Córdoba, Xavier Ferré, Nelson Medinilla, Manuel Abellanas, Pilar Herrero... También me gustaría agradecer al resto de mis compañeros de departamento, tanto profesores como PAS, así como a la gente del CTB. En especial, gracias a todos los miembros que forman y han formado parte de MIDAS - MEDAL: Ángel, Héctor, Paola, Johnny, Sergio, Marta, Nacho, Santi Muelas, Santi González, José María Peña, Dionisio, Agustín, Nicole, Miguel, Rubén, Jian, ... Todos habéis aportado algo a esta tesis, aunque sólo fueran risas y charlas en la comida.

A los profesores que me hicieron desde niño mejorar, en especial a Juan Salinero, a Jesús de Blas y a Marisa.

Los agradecimientos personales no pueden comenzar sino con el pilar fundamental de mi vida en Madrid durante los últimos 13 años: mis amigos.

A Álex, porque hemos compartido toda la carrera juntos y superado todas los baches, y ahora terminamos casi simultáneamente nuestros objetivos.

A Cherni, por ser una inspiración desde el primer año de carrera. Sigues haciéndome pensar y plantearme las cosas con cada proyecto... aunque sé que no entendemos igual estos últimos años de doctorado ;)

A Iñaki, porque a pesar de la distancia y que en los últimos años hemos perdido contacto, se te echa de menos y ojalá compartamos más momentos juntos.

A Vila, ¡qué decir! Porque has estado ahí y hemos compartido muchos buenos momentos mientras tú, persigues tu sueño y creces más y más con Witei. Este último empujón para cerrar una etapa de nuestras vidas, también teníamos que compartirlo.

A Sergio, porque te embarcaste en GAUSS y te dejaste engañar para llevarlo, y para venir a trabajar al CTB. Gracias por todo el apoyo.

A Leiva, por escucharme en uno de los momentos más difíciles y enseñarme el mundo que hay fuera.

A Jaime, porque a pesar de no haber trabajado juntos, hemos compartidos buenos momentos en estos años y espero que sigamos haciéndolo en los venideros.

A Ángel, porque compartir el sacrificio de una tesis durante todo el recorrido con alguien siempre es menos sufrido. Ya llegó mi hora de culminar la etapa que tú terminaste, y espero que podamos seguir trabajando en muchos proyectos juntos.

A Bea, porque no hace falta decir que sé que estás ahí. Aunque pasen semanas o meses sin hablar o vernos, siempre logramos encontrar el hueco para ponernos al día y compartir tantos y tan buenos momentos.

A Santi Muelas, porque descubrí un amigo con quien se puede contar y charlar de los más diversos temas. Espero que podamos seguir disfrutando de más brunches.

A Paola, porque sabes como nadie aprovechar cada oportunidad y por todas las risas, charlas y momentos compartidos. Porque se echan mucho de menos los abrazos diarios en el laboratorio que no se pueden conseguir elsewhere. 
A todo Charlie, porque aunque no hable mucho, me amenizáis los ratos muertos y sé que se puede contar con cada uno de vosotros y que hemos pasado y espero pasemos muchos momentos juntos.

A Andi, por todos los buenos momentos compartidos y porque sigamos compartiéndolos.

A mis amigos de la infancia, porque simplemente seguís ahí: Marta Ferrer, Marta Ro, Patry y Miguel.

A Patry, porque siempre has sido la madre que nos cuida a todos. Porque siempre estás ahí y me alegro que por fin se te vaya abriendo un futuro aquí cerquita para que podamos vernos más a menudo.

A Miguel, porque siempre que estamos juntos surgen conversaciones interesantes y llevamos muchos buenos momentos en la mochila. Porque sigan aumentando.

A Marta Ferrer, porque la distancia y el tiempo no importan contigo. Cada vez que nos reunimos, no ha pasado el tiempo salvo para contarnos mil anécdotas. Te deseo lo mejor para cerrar esta etapa pronto.

A Marta Ro, porque la distancia nunca es insalvable. Te echo mucho de menos y más con aquellas conversaciones nocturnas abulenses en las que nos poníamos al día. Me alegro que todo te vaya bien y espero que podamos compartir más momentos juntos.

A mi Familia, a todos, desde la mayor, mi abuela Milagros, al más pequeñín, Alejandro. Porque a la familia siempre se la tiene ahí, porque no dejáis de llenar mi vida ni un instante, y pese a todo no siempre la gente se acuerda de agradecerle todo a los familiares: primos, tíos, abuelos, padres, etc. Porque aquí también incluyo a mi familia más lejana, la de Jaén, de los que siempre llevo en un lugar especial a Amparo, porque por difíciles que sean las circunstancias siempre tienes un detalle con todo el mundo, a Santiago, porque aunque ya no estés con nosotros, sigues aquí. Descansa en paz. A mi tita Amparo, porque verte siempre me alegra el corazón.

A mi primo Fernando, porque siempre vas a tener ese hueco especial en mi corazón como mi hermano. A mi primo David porque al ser mi ahijado siempre sentiré un vínculo especial contigo. A mi prima Marta, porque como dije en mi proyecto, siempre fuiste una referencia para mí.

Por supuesto, a mis raíces más antiguas. A mi abuela Milagros porque sé que estarás muy orgullosa de tu nieto, como tu nieto lo está de ti. A mis abuelos Eusebio, Seve y Federico, porque no pasa un día que no os eche de menos y me acuerde de vosotros. Descansad en paz.

Por último, me gustaría escribir unas palabras a modo de dedicatoria de esta tesis y del trabajo realizado a las personas que más aprecio, que más me han apoyado en esta aventura y que han hecho que este camino que llega a su fin, tenga sentido. No por estar al final, son peores, sino que son las más sentidas y agradecidas.

Comienzo por tí, Héctor. Puedo decirlo más alto, pero no más claro, sin tu ayuda, sin tu apoyo, sin tus ganas de trabajar y hacer grandes cosas juntos, esta tesis no sería lo que es hoy. Estoy deseando que volvamos a trabajar codo con codo y espero que podamos compartir muchos buenos momentos juntos. GRACIAS de todo corazón.

A mi familia "postiza", empezando por Teo y $M^{a} J o s e ́$, por acogerme desde el principio y darme tanto cariño como en mi propia familia.

A Silvia, porque pese a que la distancia no nos permita disfrutar de más tiempo juntos, sé que estás ahí. 
A Marta, porque ya sabes lo mucho que te debo. Porque tengas todo lo mejor y termines tu carrera por todo lo alto, para comenzar una nueva vida que disfrutes mucho.

A Rocío, mi niña, porque hemos compartido desde el principio esta etapa que ya llega a su fin. Por aguantarme, por querer comenzar una nueva vida juntos y por apoyarme en cada paso y con cada decisión incondicionalmente. Por estar ahí en los buenos, pero sobre todo en los malos momentos. Por sufrir y celebrar juntos este hito, como haremos el resto de la vida. Te quiero.

A mis Padres, porque lo sois todo para mí. Porque gracias a vosotros toco el cielo y llego lo más lejos que se puede llegar. Por todos los sacrificios que habéis hecho por mí para que pudiera tener un pasado, un presente y un futuro que sólo gracias a vosotros me merezco. Este no es sólo mi hito y mi esfuerzo, es el nuestro. Porque os quiero y os doy las gracias de todo corazón. Esta tesis va por vosotros. 


\section{Abstract}

The increasing generation of data in different sectors thanks to digitalization has provoked a change in the techniques used to store, process and extract value and knowledge from different datasets. This change has also affected more traditional sectors like healthcare, which have been transformed in this process.

The amount of medical data generated is increasing as the adoption of Electronic Health Records (EHRs) is becoming a standard in all the developed countries. The economic impact of the digitalization of medical data is estimated at $\$ 300$ billion annually. Most of the data generated in healthcare are unstructured data: texts and images.

The focus in this thesis is on the development of new techniques and methods that allow the structure and knowledge extraction from written texts which are stored in EHRs. This data is specially relevant as it contains information on the patient's health, their signs, symptoms, treatments, diseases and their evolution. The richness of this data, which is not already being exploited, can lead to the development of information systems to help doctors to make decisions.

The main development of the research performed is focused on the analysis of the structure of the medical data mentioned in EHRs, as well as its challenges. The use of statistical models to identify the basic structures of Spanish language in written texts is the basis for the rest of developments. The identification of the different medical entities mentioned, as well as drug names, the detection of positivity or negativity of a sentence, together with the disambiguation of acronyms and abbreviations used, are some of the problems analyzed in this research.

In order to realize this analysis, an architecture named H2A: Human Health Analytics (H2A) has been designed, taking into account the interoperability of the different developed components and providing the corresponding flexibility for its use in different scenarios.

As part of the development of this thesis, the methods and techniques developed have been applied to the study of a real life use case: the analysis on the evolution of stroke patients. This use case demonstrates the potential of these methods and its applicability to scenarios in which real data is used.

Finally, in order to remark the industrial focus of this thesis, an analysis on the market conditions and a business plan has been developed to bring the technology developed in this thesis to the market.

Keywords: Natural Language Processing, Electronic Health Records, Big Data. 



\section{Resumen}

La creciente generación de datos en distintos sectores debido a la digitalización ha provocado un cambio en las técnicas para almacenarlos, procesarlos y extraer valor y conocimiento de diferentes conjuntos de datos. Este cambio ha afectado también a sectores tradicionales como el sector de la salud, que ha sufrido un proceso de transformación.

La cantidad de datos médicos generados está aumentando a medida que la adopción de las Historias Clínicas Electrónicas (HCE) se convierte en un estándar en los países desarrollados. El impacto económico de la digitalización de los datos médicos se estima en \$300.000 millones anuales. La mayor parte de los datos generados en el sector de la salud, son datos no estructurados: texto e imágenes.

El énfasis de esta tesis está en el desarrollo de nuevas técnicas y métodos que permitan la estructuración y la extracción de conocimiento de los textos escritos y almacenados en las HCEs. Estos datos cobran especial relevancia dado que contienen información sobre la salud de los pacientes, los signos, síntomas, tratamientos, enfermedades y evolución de los mismos. La riqueza de estos datos que aún no están aprovechados, puede servir para el desarrollo de sistemas de información que ayuden a los médicos a tomar decisiones.

La investigación desarrollada se centra en el análisis de la estructuración de los datos mencionados en las HCEs, así como en los retos que esto supone. El uso de modelos estadísticos para identificar las estructuras básicas del lenguaje en textos escritos en español sienta las bases del resto de los desarrollos. La identificación de los distintos términos médicos mencionados, así como los nombres de medicamentos, la detección de la positividad o negatividad de una frase, junto con la desambiguación de los acrónimos y abreviaturas usados, son parte de los problemas analizados en esta tesis de investigación.

Para la realización de estos análisis, se ha diseñado una arquitectura denominada $\mathrm{H} 2 \mathrm{~A}$ : Human Health Analytics (H2A), que permite la interoperabilidad de los distintos componentes desarrollados y que provee de la flexibilidad correspondiente para su uso en distintos casos de estudio.

Como parte del desarollo de esta tesis, se han aplicado los métodos y técnicas desarrollados en el estudio de un caso de uso real: el análisis de la evolución de pacientes de ictus. Este caso de estudio demuestra el potencial de estos métodos y su aplicabilidad en escenarios en los que se utilizan datos reales.

Finalmente, para remarcar el enfoque industrial de esta tesis, se ha realizado un análisis del mercado y un modelo de negocio para poder comercializar la tecnología desarrollada en esta tesis.

Palabras clave: Procesamiento del Lenguaje Natural, Historias Clínicas Electrónicas, Big Data. 



\section{Contents}

1 Introduction 1

1.1 Introduction and motivation . . . . . . . . . . . . . 1

1.2 Hypothesis . . . . . . . . . . . . . . . . . . 4



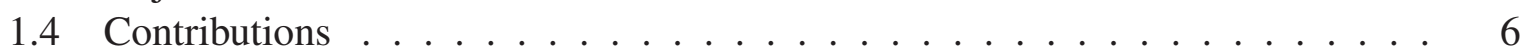

1.5 Publications, talks and recognitions $\ldots \ldots \ldots 7$

1.5.1 Journal . . . . . . . . . . . . . . . . . . . . 7

1.5.2 Reviewed Conferences . . . . . . . . . . . . . . . . 7

1.5.3 Invited talks and other workshops . . . . . . . . . . . . . . 8

1.5 .4 Internships . . . . . . . . . . . . . . . . . . . . . 9

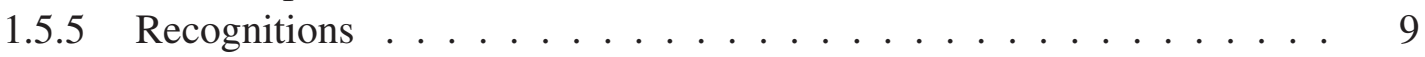

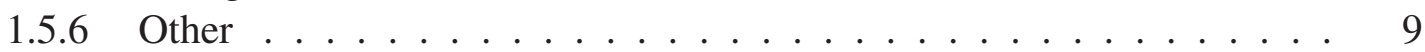

1.6 Structure of the document . . . . . . . . . . . . . . . . . . 9

2 Generic vs. domain specific corpora to apply Natural Language Processing for Spanish Electronic Health Records 11

2.1 Introduction . . . . . . . . . . . . . . . . . 11

2.2 State of the art . . . . . . . . . . . . . . . . . 12

2.2.1 Annotation standards . . . . . . . . . . . . . . . . 12

2.2.2 Training the models and existing applications . . . . . . . . . . . . . . 14

2.2.3 Availability of corpora . . . . . . . . . . . . . . . . 15

2.3 Generic vs. domain specific corpora for training models . . . . . . . . . . 15

2.3.1 Preliminaries . . . . . . . . . . . . . . . . 16

2.3 .2 Goals . . . . . . . . . . . . . . . . . . 17

2.3.3 General methodology followed in the training process . . . . . . . 20

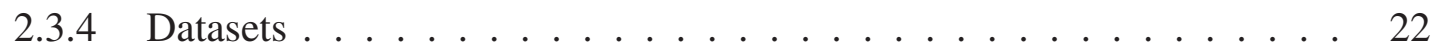

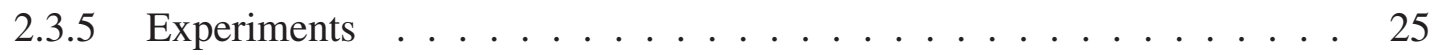

2.4 Discussion . . . . . . . . . . . . . . . . . . . 38

3 Addressing specific challenges in the application of Natural Language Processing for Spanish Electronic Health Records $\quad 40$

3.1 Introduction . . . . . . . . . . . . . . . 40

3.2 Negation . . . . . . . . . . . . . . . . . . . . 40

3.2 .1 The problem ......................... 40

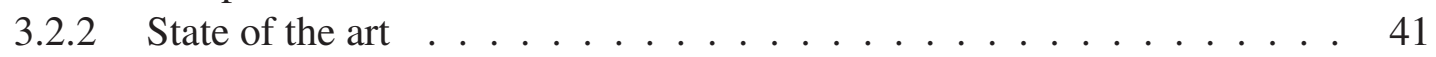

3.2 .3 Solution ........................... 42

3.3 Detection of Unified Medical Language System (UMLS) entities and drug names 47

3.3.1 The problem . . . . . . . . . . . . . . . . . 47

3.3.2 State of the art . . . . . . . . . . . . . . . 48 
3.3.3 Using MetaMap and cTAKES for English Named Entity Recognition . 51

3.3.4 Solution: Named Entity Recognition in Electronic Health Records . . . 60

3.4 Detecting medical acronyms . . . . . . . . . . . . . . . 66

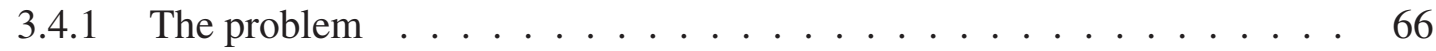

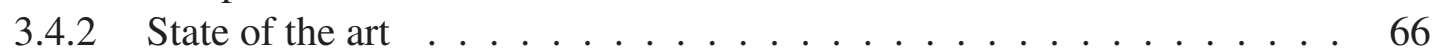

3.4 .3 Solution . . . . . . . . . . . . . . . . 68

3.5 Discussion .............................. 71

4 The architecture - H2A: Human Health Analytics 73

4.1 Introduction . . . . . . . . . . . . . . . . . . . . . 73

4.2 State of the art . . . . . . . . . . . . . . . . . . . 74

4.3 The Unstructured Information Management Architecture (UIMA) workflow . . 76

4.4 H2A: Human Health Analytics - Architecture . . . . . . . . . . . . . 78

4.4.1 Improvements proposed to the typical Natural Language Processing pipeline . . . . . . . . . . . . . . . . . 79

4.4.2 Defining the architecture . . . . . . . . . . . 80

4.4.3 Implementing the Text Analysis Framework . . . . . . . . . . . . 83

4.5 Discussion . . . . . . . . . . . . . . . . . . . . . 89

5 Applying H2A: Human Health Analytics to analyze the evolution of stroke patients 91

5.1 Introduction . . . . . . . . . . . . . . . . . 91

5.2 Background . . . . . . . . . . . . . . . . . . . . 92

5.2 .1 Analysis on strokes . . . . . . . . . . . . . . . 92

5.2.2 Neurological scales applied to stroke patients . . . . . . . . . . . 93

5.3 Extracting scale values from Electronic Health Records . . . . . . . . . . . . 95

5.3.1 Implementing the neurological scales descriptor . . . . . . . . . . . 95

5.3.2 Distribution of the scales on the different document sections . . . . . 96

5.4 Automatic identification and analysis of patients' evolution . . . . . . . . . . . 97

5.5 Implementation of an end-user application . . . . . . . . . . . . . . . . 98

5.6 Discussion . . . . . . . . . . . . . . . . . . 100

6 Commercialization: Accessing the market 102

6.1 Introduction . . . . . . . . . . . . . . . . . . . . 102

6.2 Executive summary . . . . . . . . . . . . . . . . . . 102

6.3 Strategic plan . . . . . . . . . . . . . . . . . . 104

6.4 Marketing . . . . . . . . . . . . . . . . . . 111

6.5 Operational organization . . . . . . . . . . . . . . . . 113

6.6 Goals . . . . . . . . . . . . . . . . . . . . . . . 114

6.7 Discussion . . . . . . . . . . . . . . . . . . . . . 114

7 Conclusions and future work $\quad 116$

7.1 Conclusions . . . . . . . . . . . . . . . . . 116

7.2 Future lines . . . . . . . . . . . . . . . . . . 117

$\begin{array}{ll}\text { Appendices } & 119\end{array}$ 
A IBM Watson Internship Report

Bibliography 



\section{List of Figures}

1.1 Healthcare data growth 2013 - 2020. Source: EMC Digital Universe with Research \& Analysis by IDC $[$ IDC, 2014] . . . . . . . . . . . . . . 2

2.1 A typical NLP pipeline . . . . . . . . . . . . . . . . . . . . . . 16

2.2 Flowchart describing the methodology used . . . . . . . . . . . . . 20

2.3 Description of the process followed to tune the parameters . . . . . . . . 21

2.4 Processes followed to train the models from a generic and a domain specific corpus . . . . . . . . . . . . . . . . 27

2.5 Definition of the complete process with nested CV $\ldots \ldots \ldots \ldots$

2.6 Definition of the inner $\mathrm{CV}$ to tune the parameters . . . . . . . . . . 31

2.7 Definition of the outer $\mathrm{CV}$ to build and validate the models . . . . . . . . . 32

3.1 Approach followed in the experiment of negation detection . . . . . . . . . 45

3.2 Phases of the labelling process . . . . . . . . . . . . . . . 45

3.3 Example of the UMLS coding system. Source: UMLS Online Learning . . . . 53

3.4 Architecture of the proposed solution . . . . . . . . . . . . . . 53

3.5 Comparison between statistical results for MetaMap \& cTAKES executions . . 57

3.6 Comparison of precision results for MetaMap \& cTAKES executions for each disease. . . . . . . . . . . . . . . . . . . 57

3.7 Comparison of recall results for MetaMap \& cTAKES executions for each disease. 58

3.8 Comparison of specificity results for MetaMap \& cTAKES executions for each disease. . . . . . . . . . . . . . . . . . . 59

3.9 Comparison of f1 score results for MetaMap \& cTAKES executions for each disease. . . . . . . . . . . . . . . . . . 59

3.10 Named Entity Recognition process designed . . . . . . . . . . . . . . . . 60

3.11 Parse Tree created from the sentence "se observa dilatada la arteria cerebral media derecha" . . . . . . . . . . . . . . . . . . . 61

4.1 UIMA description of the components [Ferrucci and Lally, 2004] . . . . . . . 77

4.2 UIMA's architecture $[$ Ferrucci and Lally, 2004] . . . . . . . . . . . . . . 78

4.3 A typical NLP pipeline . . . . . . . . . . . . . . . . . . . . . . . 79

4.4 The newly proposed pipeline . . . . . . . . . . . . . . . 79

4.5 An example of an extended Named Entity Recognition module . . . . . . . . . 80

4.6 Architecture developed to analyze Electronic Health Records . . . . . . . . . . 81

4.7 Text analysis framework architecture developed to structure the text written . . 83

4.8 Generic inputs/outputs of the system . . . . . . . . . . . . . . . 84

4.9 Design of H2A relational database which contains the most important annotations. 85

4.10 Project dependencies . . . . . . . . . . . . . . . . . 85

4.11 Whitespace tokenizer pipeline . . . . . . . . . . . . 86

4.12 Metrics pipeline annotators . . . . . . . . . . . . . . 87 
4.13 NLP pipeline annotators . . . . . . . . . . . . . . . . . . . 88

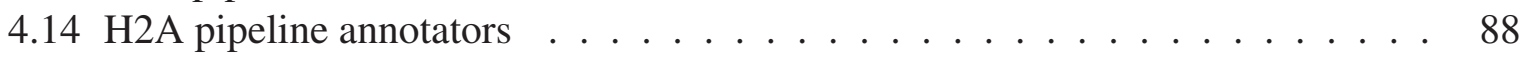

4.15 End-user application integration $\ldots \ldots \ldots$. . . . . . . . . . . . 89

5.1 Mortality rate $[$ Organization et al., 2014] . . . . . . . . . . . . . . . . . . . . 92

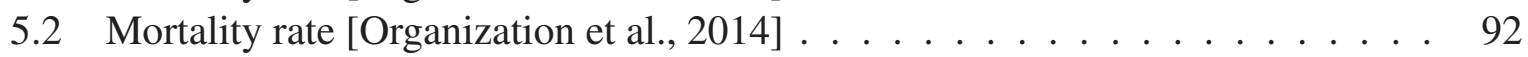

5.3 Deaths by age per year in Spain from 1980 to $2014 \ldots \ldots$. . . . . . . . . . 93

5.4 Neurological scales pipeline annotators . . . . . . . . . . . . . 95

5.5 Identified severity of the patients by age . . . . . . . . . . . . . . . . 97

5.6 General visualization of the web application . . . . . . . . . . . . 98

5.7 Selection of criteria search with search box and the sections of the documents . 99

5.8 Selection of patients for a NIHSS search . . . . . . . . . . . . . . 99

5.9 Expanded information for a patient showing the evolution of the patient in each scale . . . . . . . . . . . . . . . . . . 100

6.1 Total healthcare expenses in relation with GDP in Spain, 2000-2013. Source: Prepared by the author from OCDE, Health Data 2015, and MSSSI, Sistema de Cuentas 2013. . . . . . . . . . . . . . . . . . . . . . 105

6.2 Healthcare expenses (in euros) by age in 2010. Source: Prepared by the author from Fundación BBVA 2010 . . . . . . . . . . . . . . . . . 105

6.3 Estimation on Spain's population by age group in 2015, 2030 y 2060. Source: Prepared by the author from data from Instituto Nacional de Estadística (INE), 2015. 


\section{List of Tables}

2.1 Description of datasets $D 4_{i}(i$ from 1 to 12$) \ldots \ldots \ldots$. . . . . . . 24

2.2 Description of datasets $D 5_{j}(j$ from 1 to 5$) \ldots \ldots \ldots \ldots \ldots$

2.3 General precision, recall, F-measure and accuracy values obtained in the train-

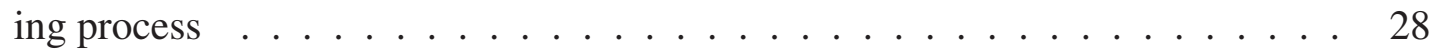

2.4 Values comparison when evaluating the models with medical texts . . . . . . 29

2.5 Experiment 2.1 - Sentence Detector parameter tuning for the different datasets . 32

2.6 Experiment 2.1 - Sentence Detector: precision, recall and F-measures for the different datasets . . . . . . . . . . . . . . . . . . . 33

2.7 Experiment 2.2 - Parameter tuning with best average accuracies for the Part-ofSpeech using both tagsets . . . . . . . . . . . . . . . . . . 34

2.8 Experiment 2.2 - Accuracies obtained for the Part-of-Speech using both tagsets in D3 and $D 4_{i}(i$ from 1 to 12$) \ldots \ldots \ldots \ldots \ldots$

2.9 Experiment 2.2 - Parameter tuning with best average F-Measures for the Chunker using both Part-of-Speech tagsets . . . . . . . . . . . . . . . 35

2.10 Experiment 2.2 - Parameters with best average F-Measures for the Chunker using both Part-of-Speech tagsets . . . . . . . . . . . . . . . . 35

2.11 Experiment 2.3 - Parameter tuning with best average accuracies for the Part-ofSpeech using both tagsets . . . . . . . . . . . . . . . . . 36

2.12 Experiment 2.3 - Accuracies obtained for the Part-of-Speech using both Partof-Speech $(\mathrm{PoS})$ tagsets in D1 and D3 . . . . . . . . . . . . 37

2.13 Experiment 2.3 - Parameters with best average F-Measures for the Chunker using both Part-of-Speech tagsets in D1 and D3 . . . . . . . . . . . .

2.14 Experiment 2.3 - F-measures obtained for the Chunker using both Part-of-Speech tagsets in D1 and D3 . . . . . . . . . . . . . . . . . 37

2.15 General Precision, Recall, F-Measure and Accuracy values obtained . . . . . . 38

3.1 Example of the translated terms and synonyms added . . . . . . . . . . . . 44

3.2 Definite Negated Terms frequencies in Spanish and English . . . . . . . . . . . 46

3.3 Probable Negated Terms frequencies in Spanish and English . . . . . . . . . . . . 46

3.4 Pseudo Negated Terms frequencies in Spanish and English . . . . . . . . . . . . 46

3.5 Correspondence between MetaMap and cTAKES semantic types . . . . . . . 54

3.6 Summary of validation sources and extraction method used . . . . . . . . . 55

3.7 Infectous diseases conforming the dataset $\ldots \ldots \ldots . \ldots . \ldots 56$

3.8 Features used to train the model. Example sentence: "Fuerza proximal MIIconservada y simétrica" . . . . . . . . . . . . . . . . . . . . . . . 69

3.9 C4.5 Confusion Matrices . . . . . . . . . . . . . . . . . . 70

$3.10 \mathrm{C} 4.5$ performance metrics . . . . . . . . . . . 70

5.1 Stroke severity depending on NIHSS value . . . . . . . . . . . . . . . . . 94 
6.1 SWOT Analysis . . . . . . . . . . . . . . . . . . . . 110

6.2 Pricing plan: only reports version (annual prices without VAT) . . . . . . . . 112

6.3 Selling plan - New customers . . . . . . . . . . . . . . . . . . 112 


\section{Listings}

2.1 Pseudocode of the cross-validation . . . . . . . . . . . . . . . . . . . . 29

3.1 Pseudocode of the NegEx algorithm . . . . . . . . . . . . . . . . . 43

3.2 Pseudocode of the ponderation of words . . . . . . . . . . . 63

3.3 Pseudocode of the ponderation of the language $\ldots \ldots \ldots$. . . . . . . 64

3.4 Pseudocode of the ponderation of the score given by Elasticsearch . . . . . . . 64

3.5 Pseudocode of the ponderation for ranking the results . . . . . . . . . . . 64

3.6 Pseudocode of the ponderation of prescriptions $\ldots \ldots \ldots \ldots$ 



\section{Acronyms}

ADL Activities of Daily Living

AEMPS Agencia Española de Medicamentos y Productos Sanitarios

API Application Programming Interface

AUI Atom Unique Identifier

BeCAS Biomedical Concept Annotation System

CAS Common Analysis Structure

CDKRM Cancer Disease Knowledge Representation Model

CNS Canadian Neurological Scale

CPE Collection Processing Engine

CT Computerized Tomography

cTAKES Clinical Text Analysis and Knowledge Extraction System

CTB Center for Biomedical Technology

CUI Concept Unique Identifier

CV Cross-Validation

CVA Cerebrovascular Accident

CVI Cerebrovascular Insult

DM Data Mining

DSS Decision Support System

EAGLES Expert Advisory Groups on Language Engineering Standards

EDSS Expanded Disability Status Scale

EHR Electronic Health Record

FN False Negative

FP False Positive

GCS Glasgow Comma Scale 
GDS Global Deteroration Scale

H2A H2A: Human Health Analytics

HCD Human Centered Design

HCE Historias Clínicas Electrónicas

HD High-Definition

HITEx Health Information Text Extraction

HPO Human Phenotype Ontology

HTTP HyperText Transfer Protocol

IBM International Business Machines

ICD International Classification of Diseases

IDE Integrated Development Environment

INE Instituto Nacional de Estadística

IP Internet Protocol

IPR Intellectual Property Rights

ISCIII Instituto de Salud Carlos III

IT Information Technologies

JCR Journal Citation Reports

JSON JavaScript Object Notation

LAG Left-Associative Grammar

LOINC Logical Observation Identifier Names and Codes

LUI Lexical (term) Unique Identifier

MaxEnt Maximum Entropy

MediTAS Medical Text Analysis System

MedLEE Medical Language Extraction and Encoding System

MeSH Medical Subject Headings

MIDAS MIning DAta and Simulation

ML Machine Learning 
MLP Medical Language Processing

mRs Modified Rankin Scale

MTENP Medical Text Extraction and NLP Procedures

MVP Minimum Viable Product

NER Named Entity Recognition

NIH National Institute of Health

NIHSS National Institute of Health Stroke Scale

NLP Natural Language Processing

NP Noun Phrase

OMIM Online Mendelian Inheritance in Man

PET Positron Emission Tomography

PoS Part-of-Speech

R\&D Research and Development

RST Rhetorical Structure Theory

SaaS Software as a Service

SD Sentence Detector

SEDOM Sociedad Española de Documentación Médica

SNOMED-CT Systematized Nomenclature of Medicine - Clinical Terms

SUI String Unique Identifier

TN True Negative

TP True Positive

TVP Term Validation Procedure

UIMA Unstructured Information Management Architecture

UMLS Unified Medical Language System

UPM Universidad Politécnica de Madrid

UX User eXperience

VTE Validation Terms Extraction Procedure 
WHO World Health Organization

XMI XML Metadata Interchange

XML eXtensible Markup Language 


\section{CHAPTER 10}

Introduction

\subsection{Introduction and motivation}

Information Technologies (IT) are experiencing a dramatic change during this decade (20112020) due to many different factors: political factors affecting cybersecurity of critical systems; industrial factors resulting in more digitalized industries; economic factors, as existing technologies are becoming cheaper and more accessible to different kind of users; and more importantly, social factors, as all kinds of people are transforming from consumers to the so called "prosumers" who are the ones that not only consume, but also produce data.

This has led to an enormous growth of data generated each single day and projections suggest that the evolution of data will multiply by a factor of 10 from 2013 to 2020, according to [IDC, 2014]. This means, that an estimated 4.4ZB of data were created in 2013, and by 2020 the amount of generated data will be around 44ZB. Other studies give the same estimations [UNECE, 2015] with 20ZB of data generated in 2017, which means data will double in just two years.

The explosion of data generated by personal devices, streaming data over Internet Protocol (IP), TV and other online media, the forthcoming autonomous vehicles, robots and other sensors, and more specifically in the medical field, the ones generated at hospitals - medical images gathered from scans, laboratory tests, emergency and discharge reports, etc. - is influencing the amount of data and the steep growth IT are experiencing. Figure 1.1 shows the increasing amount of data generated in healthcare in the following years all over the world, with a $48 \%$ annual growth rate, leading to 2,314 Exabytes of data in 2020, according to [IDC, 2014].

All this information gathered from patients, like tests performed or clinical reports written by doctors and other physicians, has been traditionally stored as part of the medical history of the patient. This information is stored just in case a healthcare professional has to come back to some piece of information. It was typically done on-site because records were written on paper, but with the digitalization of the society, hospitals and primary care centers are storing patients' information digitally in what is called an Electronic Health Record (EHR). Most relevant information in a EHR is: the patient's medical history, diagnoses, medications, treatment plans, immunization dates, allergies, radiological images, and laboratory and test results.

The adoption of EHR technology is significantly growing and is expected to continue growing in the following years [Ben-Assuli, 2015], although developed countries have adoption rates above $80 \%$. Roughly a third of US hospitals (27.6\%) had adopted EHRs back in 2011, 


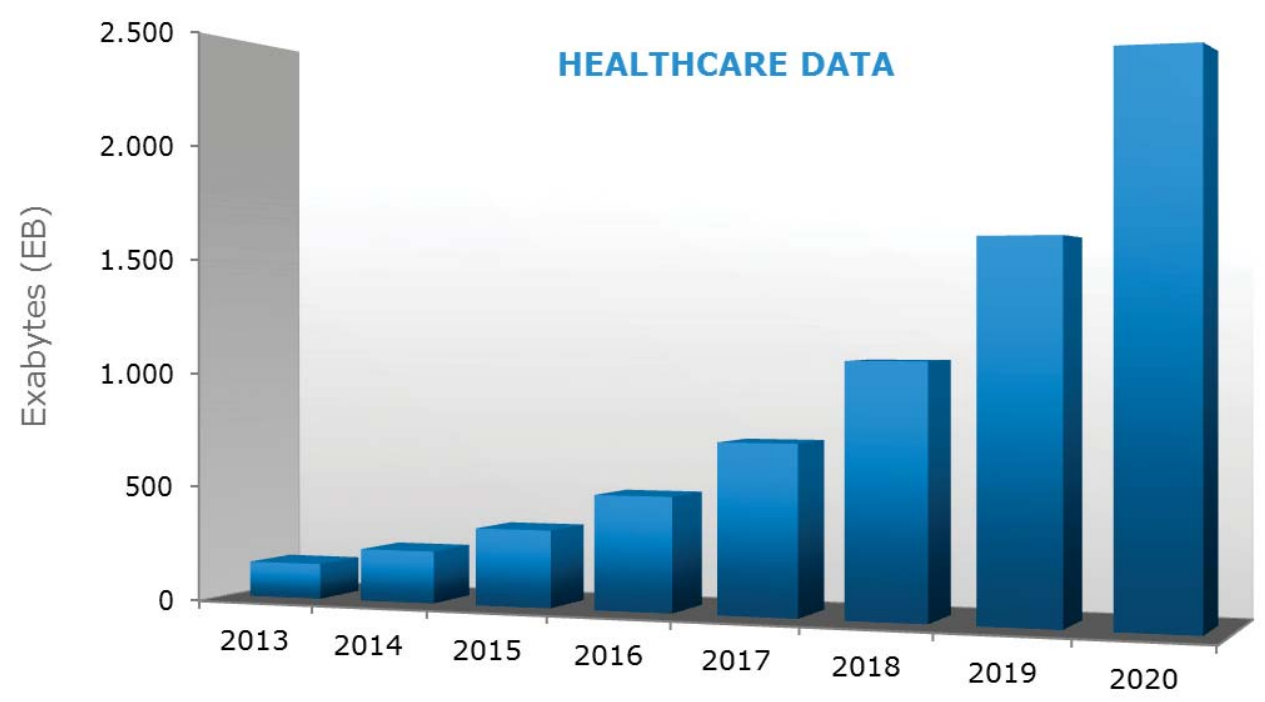

Figure 1.1: Healthcare data growth 2013 - 2020. Source: EMC Digital Universe with Research \& Analysis by IDC [IDC, 2014]

by 2015 the ratio increased to $83.8 \%$, in which more than 8 out of 10 hospitals had already implemented some kind of EHR, according to [Henry et al., 2016]. In [Accenture, 2010a], a global study performed back in 2009, Spain was in the second place on EHR adoption, with roughly $60 \%$, with projections to increase adoption to $83 \%$ by 2013 . Nordic countries seem to be on the lead on EHR adoption. Up to our knowledge no more recent global studies have been performed.

The adoption of EHR technologies has a direct impact in the expenditure for healthcare in the countries it is deployed. In [Accenture, 2010b], a forecast to reach $\$ 22.3$ billion by the end of 2015 as the global EHR market was presented. According to [Wipro, 2013] the potential annual value in the healthcare sector is estimated at $\$ 300$ billion. This amount can be detailed in different areas as follows:

- $\$ 9$ Billions to public health surveillance and response systems.

- $\$ 47$ Billions to advanced fraud detection and performance of drug pricing.

- \$5 Billions to the aggregation of data, including patient records, online platforms and different communities.

- \$165 Billions to clinical operations, having more transparency in the data obtained and the Decision Support Systems (DSSs).

- \$108 Billions in Research and Development (R\&D), personalized medicine and design of clinical trials. 
Significant advances have been made in the healthcare domain thanks to technology, allowing secure digital storage and transfer of health-related information among different industry partners. This evolution creates new sources of health information. Data analytics relies on the availability, accessibility and privacy of EHRs. Performing analytics operations on the Big Data stored in the EHRs will have a direct effect on healthcare.

These benefits cannot be achieved instantly, as technology is not mature enough to perform these analytics, due to, among other reasons, a lack of standards, a lack of interoperable data schemes, and the necessity of natural text and image processing tools.

This thesis focuses on the extraction of information from the patient's medical records written in Spanish. These records contain valuable clinical information that is written in narrative form, as part of the history of the patient or as part of the reports written after an image test is performed. In order to find relevant information in this sense, it is necessary to extract it from free-text. Other aspects, such as interoperability, ethics, or privacy are out of the scope of this work.

Natural Language Processing (NLP) is the field of Computer Science that aims to make computers able to read, understand, develop, and even interact with humans by using language, just as humans would do.

This field has been extensively studied, specially during the last decade, where the interest in the community has boosted the research made towards different kinds of its potential applications: virtual assistants (eg. Siri [Apple, 2013], Cortana [Microsoft, 2014], Google Assistant [Google, 2016]), content crawlers (ie. to extract relevant information from the web), content creation (eg. writing articles in newspapers), or even in the biomedical field (eg. to automatically annotate standardized codes for diseases) [Friedman et al., 1994], [Friedman et al., 1995], [Hohnloser et al., 1996], [Friedman, 1997], [Friedman and Hripcsak, 1999], [Aronson, 2001], [Savova et al., 2010], [Doan et al., 2014]. One of the main problems that NLP still faces today is the English-centric development in most domains, though in the last few years the research community is focusing its efforts to create common structures and methods to perform language analysis independently of the language, or even to produce cross-language resources.

Despite being Spanish one of the most used languages with around 500 million speakers worldwide (according to [Cervantes, 2016]), there are few resources to automatically extract relevant information from unstructured texts written in this language, particularly talking about the adaptation to healthcare. Existing efforts in other languages have been made, more typically in German, French or Japanese, but the application of these techniques in other languages are futile without proper work to adapt the algorithms to the terminology used in Spanish.

In the case of creating models for NLP, supervised learning is the most common approach. Worst counterpart of using supervised learning methods is that a complex annotation process has to be done in order to achieve the desirable results. This process requires human intervention and it is very costly, as creating the corpora for annotating Part-of-Speech (PoS) and phrases has to be performed. In order to create this type of annotations for the machine to learn, a common set of tags have to be used [EAGLES Computational Lexicons Working Group, 1996], [Petrov et al., 2011], [Nivre et al., 2015]. In the case of general purpose models 
that are not very specific for a particular domain, already collected corpora can be used [Moreno et al., 2000], [Martí et al., 2007a], [da Cunha et al., 2011], [Marimon et al., 2014].

Medical texts contain a lot of very specific information for this particular domain. Moreover, there is a challenge in using generic corpora in systems able to understand and analyze clinical data extracted from Spanish written texts. The comparison of generic corpora vs. specific ones for a particular domain, or the use of the former to specific tasks in different NLP have already been studied [Vogel, 2003], [Agirre et al., 2009]. This thesis also addresses this problem and introduces a tool to annotate corpora, which has been used to create an annotated medical corpus from real world data. This corpus has been used to train, test and deploy models that are able to understand very complex terminology used in EHRs. A comparison of the performance of generic and specific domain corpus has also been performed.

The main goal of this thesis though is to provide users with a comprehensive and complete NLP pipeline and complementary services to process and structure medical texts contained in EHRs. Such a service would grant end-users, experts in healthcare, access to applications which are friendly and let them exploit and analyze the data they want to. Therefore, end-user applications have to be developed in the different collaborations for the experts to be able to analyze the structured data that has been generated in the processing of the unstructured texts used as a source. The potential impact on the availability of these type of systems is analyzed throughout the thesis and the feasibility of the technology is put under test in a real life scenario.

The NLP pipeline developed in this thesis is based on Unstructured Information Management Architecture (UIMA) [Ferrucci and Lally, 2004], an architecture created by International Business Machines (IBM) to be used as a common platform in which to annotate and structure different kinds of unstructured data like texts, images, video, or voice. The use of UIMA will make it possible for the different components to communicate. UIMA creates XML Metadata Interchange (XMI) file annotations which are interoperable and stored as eXtensible Markup Language (XML) files. These type of annotations make possible that different modules interchange data and not only consume, but also produce new annotation types.

This research has been performed at the MIning DAta and Simulation (MIDAS) research group located at Center for Biomedical Technology (CTB), Universidad Politécnica de Madrid (UPM). This environment has successfully created synergies with hospitals which are the data owners in this particular case. A tighter collaboration has been created to obtain the best results with different public hospitals, collaborating in the development of this research by giving knowledge and data in the domains of oncology and neurology. The involvement of other institutions and domain experts in those fields has been key for the development of the thesis.

\subsection{Hypothesis}

The hypothesis that originate the work presented in this thesis are:

Hypothesis 1 (H1): Natural Language Processing techniques can be used to extract relevant in- 
formation without human intervention from unstructured texts like those written in Spanish Electronic Health Records.

Hypothesis 2 (H2): Models created using generic corpora can have a predictive value similar to the ones generated with domain specific corpora.

Hypothesis 3 (H3): Extraction of particular entities tied to a domain like healthcare can improve the detection of hidden knowledge in the EHRs.

\subsection{Objectives}

The main goal of this thesis is:

"To analyze, design, and develop intelligent platforms to query Electronic Health Records written in Spanish."

In order to fulfill this goal, the following subgoals have to be achieved:

\section{Objective 1 (O1)}

"To train, test, and deploy computational models for Natural Language Processing in Electronic Health Records written in Spanish language."

This objective wants to analyze the effectiveness and performance of generic vs. domain specific corpora applied in the healthcare domain.

\section{Objective $2(\mathrm{O} 2)$}

"To design, implement and test new procedures to tackle the specific problems in analyzing EHRs Spanish written texts, such as negation detection, Named Entity Recognition (NER), or acronyms disambiguation."

Objective 2 is achieved by developing innovative solutions to the aforementioned problems which are common to the NLP analysis of EHRs. 


\section{Objective $3(\mathbf{O 3})$}

"To design a domain independent architecture to query unstructured documents in order to extract meaningful information."

This objective solves the problem of creating an architecture that is independent of the domain to be used, focused on the analysis of textual unstructured documents, and with the ability to provide end-users with applications to exploit the annotated data.

\section{Objective 4 (O4)}

"To deploy the architecture in the medical domain testing it in a real life scenario."

Objective 4 deploys the architecture in a use case with real data from patients suffering a stroke.

\section{Objective 5 (05)}

"To develop a market analysis and a business plan in order to commercialize the technology."

This objective aims to the creation of a business plan to deliver this architecture to the healthcare market.

\subsection{Contributions}

The contributions featured in the development of this thesis are the following:

- The design of an architecture, based on the UIMA framework, to join the main components developed in a common framework in order to structure and query clinical data. All these modules are domain independent and can be used and developed for a general purpose, ensuring that the effort made in their development will make the system usable in many different contexts. Some of the modules, are inevitably dependent on the domain in which the application is deployed, but the architecture ensures that independently of the sources of data used, the communication of the domain-independent and the domain-dependent is transparent so the change of the domain can be done effortlessly. The architecture implements most of the modules using the latest available technology and complying with the standards so the interoperability is also ensured. 
- The implementation of a NLP pipeline that allows preprocessing of the textual data, the annotation of different syntactical information, and the extraction of domain-dependent information. The core pipeline is generic enough to analyze different kinds of text, but expandable to plug-in different modules to understand more specific domain terminology.

- The specific models created for the core NLP pipeline to be applied in the medical domain. These models have been created by manually annotating data and training using Maximum Entropy (MaxEnt) algorithms implemented in OpenNLP [OpenNLP, 2011].

- The creation of specific annotations using the UIMA framework for domain specific information. These annotations, like the doses for a particular drug mentioned in the treatment for a patient, the different values obtained in a laboratory test, or more significantly, the values of the different scales used in neurology for determining the severity of the patients that have suffered a stroke.

- The creation of a standardized database for drugs containing information retrieved from Agencia Española de Medicamentos y Productos Sanitarios (AEMPS) ${ }^{1}$.

- The development of an end-user application that has been created for and with domain experts in the field of neurology, to make sure that the data processed is available to the end-users that really need to exploit it. This application has been built following a Human Centered Design (HCD) to maximize the User eXperience (UX), focusing on its usability and performance.

\subsection{Publications, talks and recognitions}

The results presented in this thesis are documented in the following publications:

\subsubsection{Journal}

- Roberto Costumero, Jesús Sánchez, Ángel García-Pedrero, Diego Rivera, Mario Lillo, Consuelo Gonzalo-Martín, and Ernestina Menasalvas. Geography of legal water disputes in Chile. Journal of Maps 13(1), 7-13, 2017. DOI: 10.1080/17445647.2016.1252803.

- Diego Rivera, Alex Godoy-Faúndez, Mario Lillo, Amaya Alvez, Verónica Delgado, Consuelo Gonzalo-Martín, Ernestina Menasalvas, Roberto Costumero, and Ángel GarcíaPedrero. Legal disputes as a proxy for regional conflicts over water rights in Chile. Journal of Hydrology, 535:36-45, 2016. DOI: 10.1016/j.jhydrol.2016.01.057

- Alejandro Rodríguez-González, Roberto Costumero, Marcos Martinez-Romero, Mark D.Wilkinson and Ernestina Menasalvas-Ruiz. Extracting Diagnostic Knowledge from

\footnotetext{
${ }^{1}$ Source of the information: Agencia Española de Medicamentos y Productos Sanitarios http://www . aemps.gob.es
} 
MedLine Plus: a Comparison between MetaMap and cTAKES Approaches. Current Bioinformatics. Accepted, yet to be published.

\subsubsection{Reviewed Conferences}

- Roberto Costumero, Ángel García-Pedrero, Consuelo Gonzalo-Martín, Ernestina Menasalvas, and Socorro Millan. Text analysis and information extraction from Spanish written documents. In International Conference on Brain Informatics and Health, pages 188-197. Springer International Publishing, 2014. DOI: 10.1007/978-3-319-09891-3_18

- Roberto Costumero, Angel Garcia-Pedrero, Isaac Sánchez, Consuelo Gonzalo, and Ernestina Menasalvas. Electronic Health Records Analytics: Natural language processing and image annotation. BIG DATA AND APPLICATIONS, page 1, 2014.

- Roberto Costumero, Consuelo Gonzalo, and Ernestina Menasalvas. TIDA: A Spanish EHR semantic search engine. In 8th International Conference on Practical Applications of Computational Biology \& Bioinformatics (PACBB 2014), pages 235-242. Springer, 2014. DOI: $10.1007 / 978-3-319-07581-5 \_28$

- Roberto Costumero, Federico Lopez, Consuelo Gonzalo-Martín, Marta Millan, and Ernestina Menasalvas. An approach to detect negation on medical documents in Spanish. In International Conference on Brain Informatics and Health, pages 366-375. Springer International Publishing, 2014. DOI: 10.1007/978-3-319-09891-3_34

- Alejandro Rodríguez-González, Marcos Martínez-Romero, Roberto Costumero, Mark D Wilkinson, and Ernestina Menasalvas-Ruiz. Diagnostic knowledge extraction from MedlinePlus: An application for infectious diseases. In 9th International Conference on Practical Applications of Computational Biology and Bioinformatics, pages 79-87. Springer International Publishing, 2015. DOI: 10.1007/978-3-319-19776-0_9

- Ernestina Menasalvas, Alejandro Rodriguez-Gonzalez, Roberto Costumero, Hector Ambit, and Consuelo Gonzalo. Clinical narrative analytics challenges. In International Joint Conference on Rough Sets, pages 23-32. Springer International Publishing, 2016. DOI: 10.1007/978-3-319-47160-0_2

- Gemma Reig Roselló, Roberto Costumero, Héctor Ambit, Consuelo Gonzalo, Ernestina Menasalvas, Santiago Trillo Senin, Jorge Gómez Zamora, Guillermo Fernández, Gustavo Zapata Wainberg, José Vivancos Mora. Aplicación de la computación cognitiva para el análisis de datos masivos (Big Data) extraídos de los informes de alta de una unidad de ictus. In LXVIII Reunión Anual Sociedad Española de Neurología, 2016.

- Ignacio Rubio-López, Roberto Costumero, Héctor Ambit, Consuelo Gonzalo-Martín, Ernestina Menasalvas, and Alejandro Rodriguez-Gonzalez. Acronym disambiguation in Spanish Electronic Health narratives using machine learning techniques. Studies in health technology and informatics, pages 251, 255. IOS Press, 2017. DOI: 10.3233/978$1-61499-753-5-251$ 


\subsubsection{Invited talks and other workshops}

- Roberto Costumero. Relación universidad - industria del futuro: Watson. Guide Share Europe - GSE Reunión Española. December, 15th, 2015. IBM Client Center, Madrid, Spain.

- Roberto Costumero. Unveiling the power of Cognitive Systems: Watson for future professionals. Guide Share Europe - GSE Executive Club. April, 4th - 5th, 2016. Westin Palace, Madrid, Spain.

- Roberto Costumero. Usage of NLP in H2A: Human Health Analytics to extract information from Electronic Health Records. PhD symposium at Try IT! 2017. March, 15th, 2017. E.T.S. Ingenieros Informáticos, Madrid, Spain.

\subsubsection{Internships}

- During the development of the thesis, the author did an internship at IBM Ireland dependencies to join the IBM Multilingual Watson Core team based in Dublin, Ireland, as part of the international certification at the end of the $\mathrm{PhD}$. studies. During the internship the author helped the team in improving of Part-of-Speech tagging in Spanish, improving the Dependency Parsing also in Spanish, analyzing and improving the overall performance of Watson by developing or proposing new approaches. The details of the internship and the complete original document can be found in Appendix A.

\subsubsection{Recognitions}

- One of the best 10 business ideas for H2A: Human Health Analytics, at XII Competición de Creación de Empresas, ActúaUPM, in March 2015, leading to the automatic access to the second phase of the competition.

- Finalist as one of the best business plans for H2A: Human Health Analytics presented at XII Competición de Creación de Empresas, ActúaUPM, in December 2015.

\subsubsection{Other}

There are other documents assessing some of the results obtained during the development of this thesis, but due to their confidential status have not been disclosed.

- Complete original business plan presented at ActúaUPM XII edition and kept by CAIT center at Universidad Politécnica de Madrid.

- Source code that is kept confidential as an asset and is on the process of being officially registered by Universidad Politécnica de Madrid as its owner. 


\subsection{Structure of the document}

The rest of this thesis is organized as follows:

- In Chapter 2, an analysis on the performance of NLP models trained with generic vs. domain specific corpora for Spanish using OpenNLP is addressed.

- In Chapter 3, the main specific challenges in the application of NLP to EHRs are analyzed: negation, NER, and acronym disambiguation.

- In Chapter 4, H2A: Human Health Analytics (H2A), a domain independent architecture to extract information from unstructured texts is explained in detail.

- In Chapter 5, the application of $\mathrm{H} 2 \mathrm{~A}$ architecture to a real use case in the healthcare domain is explained.

- In Chapter 6, a possible commercialization plan for the software developed is explained.

- Finally, Chapter 7 shows the contributions, conclusions and future possible research topics to be developed. 


\section{CHAPTER \\ 2}

\section{Generic vs. domain specific corpora to apply Natural Language Processing for Spanish Electronic Health Records}

\subsection{Introduction}

Statistical methods have proved to be an effective way when dealing with the understanding of human language. The training of statistical models needs corpora annotated with metadata to assign each element its corresponding class. Therefore, the main challenge to deal with in the application of NLP techniques to EHR, or any other domain specific set of documents, is to find or to generate the proper annotated corpora to be used.

Natural Language Processing can be defined as the process of automatically extracting information from written texts using a pipeline of components developed for particular tasks: I) Sentence Detector (SD), to determine the scope of sentences and separate relevant pieces of information in minimal scope entities; II) PoS tagging, in which each token is assigned to its corresponding function (nouns, verbs, adverbs, adjectives, etc.); and III) Chunker (also called Shallow Parsing) in which tokens are joined in the different phrases found in each sentence, according to their PoS. For each of the tasks, NLP needs annotated corpus in order to generate statistical models to interpret what is written on the text.

The annotation of the corpora has to be as accurate and relevant as possible. This process is typically done as a manual task, as language ambiguity makes it hard to perform automatic annotation. This annotation process is done with the use of tagging standards, to improve the quality of the process. The majority of them are defined for the English language [Santorini, 1990], although some efforts have been made to define a universal multilingual set of tags [Petrov et al., 2011]. There is also the argument that the more specific an annotated corpus is to a specific domain, the better the results that we are going to get [Drouin, 2004].

Therefore, the lack of domain specific annotated corpora created for their use in EHRs suppose a problem when applying NLP techniques in the healthcare domain. In this chapter, this challenge is addressed and the problem is solved by creating our own annotated corpus with reports from EHRs. A complete analysis on the influence of the generic corpora and the domain specific corpus created is done by determining the performance of the models created to be used with EHRs. 
Therefore, the rest of the chapter has been organized as follows: Section 2.2 exposes the kind of corpora available and the methods used to train statistical models. Moreover, there are some resources available in other languages, specially in Spanish, those resources lack the specificity of the studied domain and may not be sufficient to develop new models to successfully apply NLP to EHRs. In Section 2.3, we face the problem in order to analyze the performance of the trained models with generic and domain specific corpora. We present a study developed using different datasets to train and test models for the different NLP components using OpenNLP [OpenNLP, 2011].

This chapter closes with a discussion in Section 2.4 with the advantages and disadvantages of the solutions proposed.

\subsection{State of the art}

This section focuses on the one hand in analyzing the existing standards for annotating documents to create annotated corpus, and on the other hand on reviewing the state of the art for the problems mentioned regarding the availability of trusted resources in order to train the statistical models. To sum up, a review on the existing annotated corpora is done.

\subsubsection{Annotation standards}

Corpus annotation is the practice of adding interpretative linguistic information to a corpus generating added value. This information is typically done by adding metadata to the text being processed. The metadata included in the texts to be analyzed in natural language are called tags. These tags have to be concise and unambiguous in representing what they stand for. However, different schemes have arisen.

The Brown Corpus was the first computer-readable general corpus of texts prepared for linguistic research into modern English [Francis and Kucera, 1979]. The first version highlighted 87 simple tags giving rise to compound tags. The Brown Corpus has inspired a whole family of corpora, including the Lancaster-Oslo/Bergen Corpus (LOB) [Leech et al., 1986], Brown's British English counterpart, as well as Frown [Hundt et al., 1999] and FLOB, the 1990s equivalents of Brown and LOB respectively. The Lancaster-Oslo/Bergen (LOB) Corpus uses about 135 tags and the Lancaster UCREL group about 165 tags.

In [Marcus et al., 1993], the construction of an annotated corpus called the Penn Treebank, consisting of over 4.5 million American English words is detailed. During the first three-year phase of the Penn Treebank Project (1989-1992), the corpus was annotated with PoS information. The Penn Treebank tagset [Santorini, 1990] was used for this annotation. The Penn Treebank tagset is based on that of the Brown Corpus. However, the stochastic orientation of the Penn Treebank and the resulting concern with sparse data have led to modify the Brown Corpus tagset by paring it down considerably. A key strategy in reducing it was to eliminate redundancy by taking into account both lexical and syntactic information. Thus, whereas many 
PoS tags in the Brown Corpus are unique to a particular lexical item, the Penn Treebank strives to eliminate these instances of lexical redundancy.

In the early 1990s, the European Union launched an initiative under the name of Expert Advisory Groups on Language Engineering Standards (EAGLES) with the aim of encouraging the standardization of practices for natural language processing in academia and industry, particularly but not exclusively in the EU. One group of experts set to work on corpora, and from this and later initiatives, several documents specifying guidelines (or provisional standards) for corpus annotation emerged.

Mark-up-based annotations are gaining popularity as a result of some advantages, such as the possibility of interchanging documents, including corpora, between users or research sites. On the other hand, the use of the mark-up language itself can be efficiently parsed or validated, enabling the annotator to check whether there are any ill-formed traits in the markup, which would signal errors or omissions.

However, one drawback of these mark-up languages is that they tend to be more "verbose" than the earlier symbolic conventions used (Brown or LOB corpora).

Within the overall Standard Generalized Markup Language (SGML) framework, different co-existing encoding standards have been proposed such as the Corpus Encoding Standard (CES) recommended as an EAGLES standards [Ide, 1998]. The CES [Ide, 1998] is a part of the EAGLES Guidelines that provides a set of encoding standards for corpus-based work in natural language processing applications.

In [Ide et al., 2000], the CES has been instantiated as an XML application called XCES. Conversion to XML enables the use of some of the more powerful mechanisms provided in the XML framework, including the XSLT Transformation Language, XML Schemes, and support for inter-rescue reference together with an extensive path syntax for pointers.

There is a tagset that consists of twelve universal PoS categories proposed in [Petrov et al., 2011] to standardize best-practices. In addition to the tagset, the authors have developed a mapping from 25 different treebank tagsets to this universal set. As a result, when combined with the original treebank data, this universal tagset and the mapping produces a dataset consisting of common PoS for 22 different languages. Behind this project underlies the idea that there is a set of (coarse) syntactic PoS categories in similar forms across the languages.

In fact in [Das and Petrov, 2011] the authors propose to define manually a mapping from language and treebank specific fine-grained tagsets to a predefined universal set for those cases in which corpora with common tagsets are unavailable. The authors present both the approach and the results of the evaluation of their cross-lingual PoS projection system for six different languages.

The main experts in NLP, corpus annotation, and statistical modeling training have created in a joint effort the Universal Dependencies [Nivre et al., 2015], [Nivre et al., 2016], a multilingual treebank collection and a new generic tagset and method for annotating corpora that tries to solve the main problems regarding multilingual environment: lack of resources, ambiguity and interoperability. This sets a new "de facto" standard so new applications and 
Chapter 2. Generic vs. domain specific corpora to apply Natural Language Processing for Spanish Electronic Health Records

2.2. State of the art

methods for training models are agnostic of the language. De facto standards encapsulate what people have found to work in the past, which suggest that they should be adopted by people undertaking a new research project, so as to support a growing consensus in the community.

The creation of these standards to annotate the documents used in the datasets for training models help in the standardization of the NLP processes across different languages and domains.

\subsubsection{Training the models and existing applications}

One of the most common software platforms used to train statistical models using customized sets of tags is Apache OpenNLP [OpenNLP, 2011], which provides different tools in order to train Sentence Detection, Tokenizer, Part-of-Speech, Chunker or Named Entity Recognition, just to name a few. The training of the models in OpenNLP is carried out in a simple way and each of the components to be trained requires a set of parameters that have to be tuned in order to obtain the best possible results, generating a model that can be later used in the training of the models.

The use of the aforementioned standards in EHR and using a software like Apache OpenNLP, could serve to the creation of models to extract valuable clinical information contained in patient medical records that is expressed as free-text written in narrative form. To find relevant information it is often necessary to extract it from the free-texts in EHRs to support clinical an research processes. This information can then be associated to standard terminology concepts. Meystre et al. [Meystre et al., 2008], analyze different uses of information extraction from textual documents in EHR. According to them, information extraction from clinical texts points to a special challenge because, for instance, they contain telegraphic and shorthand phrases, abbreviations, acronyms and spelling errors.

Regarding the use of NLP in the healthcare domain, there are some projects which are worth mentioning when talking about the training of statistical models to analyze EHRs in languages different than English. Some of these systems have been developed to process clinical text in German [Hohnloser et al., 1996], [Pietrzyk, 1991]. An approach called Left-Associative Grammar (LAG) was used in MediTas [Pietrzyk, 1991], to parse summary sections of cytopathological findings reports for a Medical Text Analysis System for German. For the German SNOMED II version another NLP parser is presented in [Hohnloser et al., 1996]. The parser divides a medical term into fragments which might contain other SNOMED terms. These projects show that, although scarce, there are resources in different languages for the medical domain and there is an interest in developing these kind of methods. As cited in Savova et al. [Savova et al., 2010], there are no community resources such as annotated medical corpus in the clinical domain, so in the evaluation on tools like cTAKES, own corpus has been developed. Using the gold standard linguistic annotations of Penn TreeBank (PTB) [Marcus et al., 1993] and GENIA corpus [Kim et al., 2003] together with their own Mayo Clinic EMR corpus, cTAKES models were trained. The lack of corpus in Spanish language for the healthcare domain makes such training difficult these days. 
Chapter 2. Generic vs. domain specific corpora to apply Natural Language Processing for Spanish Electronic

\subsubsection{Availability of corpora}

Although Spanish has nearly 500 million speakers worldwide, and despite being positioned in the second place of languages per number of speakers [de la Concha et al., 2013], the availability of these resources is scarce. In this sense, tools to extract medical information from Spanish EHR are practically non existent. Even though, there is a multilevel annotated corpus ${ }^{1}$, which is available for Catalan and Spanish known as AnCora (ANotated CORporA) [Aparicio et al., 2008], [Martí et al., 2007b], [Recasens and Martí, 2010], [Taulé et al., 2008]. AnCora has more than half a million words useful for testing NLP systems. The latest version (3.0) has 517,195 words in 17,375 sentences. The 3LB-CAT/ESP corpora [Palomar et al., 2004], [Taulé et al., 2004] was expanded and enriched with semantic information in order to generate AnCora.

Other approaches for creating Spanish corpora are presented in [Moreno et al., 2000], [da Cunha et al., 2011], and [Marimon et al., 2014]. IULA Spanish LSP Treebank is another completely annotated Treebank corpus with 42,000 sentences and almost 590,000 tokens. This corpus uses texts written in the fields of Law, Economy, Genomics, Medicine, and Environment, including more generic texts from newspapers.

Based on the Rhetorical Structure Theory (RST) [Mann and Thompson, 1988], the development of a RST Spanish Treebank is presented in [da Cunha et al., 2011]. This corpus contains 52,746 words and 267 specialized texts divided in three levels: high level (doctoral theses and scientific articles), average level (textbooks), and low level (magazines, websites, ...). The texts are divided in different domains: Astrophysics, Earthquake Engineering, Economy, Law, Linguistics, Mathematics, Medicine, Psychology and Sexuality.

In [Moreno et al., 2000], an analysis of the application of Treebank resources to parsing is performed. In this work, the corpus presented contains 1,500 annotated sentences with 22,695 words gathered from the online edition of El País newspaper and the magazine Compra Maestra.

Having such resources is important for the analysis on the performance of these models when applied to the EHRs. However, the only known study to create a clinical corpus in Spanish [Marimon et al., 2017] is very recent (April, 2017) that could serve for creating NLP models for Spanish written documents in the healthcare sector, nor an study on the comparison of the performances between generic corpora vs. specific one has been made. There are some studies [Vogel, 2003], [Agirre et al., 2009], that have pointed in that direction using English documents.

\subsection{Generic vs. domain specific corpora for training models}

In this section the approach followed to solve the problem of the scarcity of the resources in the healthcare sector for performing NLP in Spanish written documents is described. The experiments conducted and their results are also analyzed.

\footnotetext{
${ }^{1}$ http: //clic.ub.edu/corpus /
} 
Chapter 2. Generic vs. domain specific corpora to apply Natural Language Processing for Spanish Electronic Health Records

2.3. Generic vs. domain specific corpora for training models

First, in order to better understand the process followed, some required definitions for the understanding of the process are expressed. Afterwards, the general methodology and the results of the experimentation performed are explained, alongside with the description of the different tagsets used and the datasets used in the experiments. Finally, the different experiments performed are analyzed.

\subsubsection{Preliminaries}

For the purpose of generating models to perform Natural Language Processing tasks, Apache OpenNLP [OpenNLP, 2011] library has been used to train the different models in a NLP pipeline. This library supports the most common NLP tasks, such as tokenization, sentence segmentation, Part-of-Speech tagging, named entity extraction, chunking, parsing, and coreference resolution.



Figure 2.1: A typical NLP pipeline

Each of these individual tasks are typically used forming a pipeline (see Figure 2.1) in order to perform advanced automatic understanding of texts. The components typically trained are the following:

- Sentence Detector: OpenNLP Sentence Detector creates models that statistically determine if a punctuation character marks the end of a sentence or not. A sentence is defined as the longest white space trimmed character sequence between two punctuation marks, except the first and last sentences in a text which are inherently different. The first non whitespace character is assumed to be the begin of a sentence, and the last non whitespace character is assumed to be a sentence end.

Typically, sentence detection is the first step in NLP and it is usually performed before doing the tokenization of the text.

- Tokenizer: The tokenizer splits a given input character sequence into the different tokens that conforms it. Typically this is performed per sentence as an independent task. These tokens constitute the "minimum pieces of information" in a sentence and they are usually words, punctuation symbols, numbers, etc.

- Part-of-Speech tagger: The Part of Speech Tagger assigns tags to the tokens to define their corresponding word type (depending on their syntactic function) based on the token itself and the context in which the token is. A token might have different possible PoS tags depending on the kind of token and its context, although the tagging process should give a unique outcome. 
Chapter 2. Generic vs. domain specific corpora to apply Natural Language Processing for Spanish Electronic

This tagger uses a probability model to predict the correct PoS tag out of a given tagset previously defined. To limit the possible tags for a given token, a tag dictionary can be used to increase tagging and runtime performance of the tagger.

- Chunker (also known as Shallow Parsing): Text chunking consists of dividing a text in syntactically correlated parts of words, like noun phrases, verb phrases, but does not specify their internal structure, nor their specific role in the main sentence.

- Parser: Determines a tree of the syntactical meaning of a phrase, dividing it into subject, verb and objects and determining the relationship among them.

- Named Entity Recognition: Typically performed at the end, but could be executed after shallow parsing, defines tokens or phrases with their corresponding semantical meanings or categories, allowing the algorithm to "understand" the meaning of the words written.

\subsubsection{Goals}

This chapter focuses on the analysis of the performance of statistical models generated from generic corpora against those generated with domain specific corpora. In this case, the focus is on the analysis on their performance in the healthcare domain.

In order to train the models, a generic annotated Spanish corpus has been used and some in-house developed corpora have been created to compare their performance.

The evaluation performed consists on:

- Comparing the performance of the models generated with the generic corpus with those from the domain specific corpus. In this case, the generic corpus is much greater than the domain specific one.

- Comparing the performance of sentence detection with models generated with the generic corpus and a larger domain specific one.

- Comparing the performance of PoS tagging and Chunker with models generated from a generic corpus and a domain specific corpus which are equally sized.

- Comparing the performance of PoS tagging and Chunker with models generated with both corpora with different tagging standards. This allows a comparison with more detailed semantic information between a large generic corpus and a small domain specific one.

To generate these models different tagsets have been used. In the following subsections, the tagsets for PoS and Chunker are explained. Finally, the evaluation metrics used in the overall process are detailed. 
Chapter 2. Generic vs. domain specific corpora to apply Natural Language Processing for Spanish Electronic Health Records

2.3. Generic vs. domain specific corpora for training models

\section{Description of the Part-of-Speech tagsets}

The training of NLP models typically relies on the annotation of corpora in order to apply supervised or semi-supervised learning algorithms. The annotation of these corpora can be made in many different ways, but the most common is to use a previously-defined tagset. These tagsets are typically designed to make the Part-of-Speech annotation that is the most complex part of the learning process, and the one with most kinds of tags to be assigned to each token.

In the experiments performed, two different tagsets have been used: i) the Universal PoS tagset [Petrov et al., 2011] and ii) the EAGLES based tagset [EAGLES Computational Lexicons Working Group, 1996].

Universal PoS tagset In order to get a more generic annotation this tagset was developed by gathering different tagsets and reaching a consensus on the generic types that a tagset should have.

The categories defined are these:

1. Adjectives - ADJ

2. Adverbs - ADV

3. Determiners - DET

4. Nouns - NOUN

5. Verbs - VERB

6. Pronouns - PRON

7. Conjunctions - CONJ

8. Adpositions (prepositions and postpositions) - ADP

9. Particles or other function words - PRT

10. Punctuation symbols - .

11. Numerals - NUM

12. Other (including typos and abbreviations) - X

EAGLES Tags defined in the EAGLES standard generate different combinations of letters to define the main category of a given token and to assign more semantic information.

For example, for a given adjective the corresponding tag can associate whether it is qualifying or not, if it is diminutive, its gender, number or even if it is a verb working as an adjective. These features are put into the tags and may vary between the different tags.

The different categories defined are as follows, where the first letters are unique to the type of tags. These tags are:

1. Adjectives $-\mathrm{a}+$ five qualifying features

2. Adverbs $-r+$ one qualifying feature 
Chapter 2. Generic vs. domain specific corpora to apply Natural Language Processing for Spanish Electronic

3. Determiners $-\mathrm{d}+$ five qualifying features

4. Nouns $-\mathrm{n}+$ six qualifying features

5. Verbs $-v+$ six qualifying features

6. Pronouns $-p+$ seven qualifying features

7. Conjunctions $-\mathrm{c}+$ one qualifying feature

8. Interjections - $\mathrm{i}$

9. Prepositions $-\mathrm{sp}+$ three qualifying features

10. Punctuation symbols $-\mathrm{f}+$ one or two qualifying features

11. Numerals $-\mathrm{z}+$ one qualifying feature

12. Dates and times - w

Although these tags have variable values, these values are tabulated and are limited for each type of tag.

\section{Description of the Chunker tagset}

The training of the models regarding the phrases that a token corresponds to, needs the use of a particular tagset similar to the ones used for the Part-of-Speech training.

In the experiments performed, the tagset used for the Chunker models is composed of the following tags:

1. Nominal Phrases - NP

2. Adjectival Phrases - AP

3. Verbal Phrases - VP

4. Adverbial Phrases - RP

5. Others (not fitting other category)- $\mathrm{O}$

Each of these tags, have prepended a B, if the token is the first one in the phrase, or an I, if the token is not the first in the phrase. For example, in a nominal phrase composed of an article and a noun, the article will have the tag B-NP, while the noun will have the tag I-NP.

\section{Evaluation metrics used to compare the models generated}

For analyzing the results of the classifiers the standard precision, recall, accuracy and F-measure as described in the following equations, using True Positives (TP), True Negatives (TN), False Positives (FP) and False Negatives (FN), have been used: 
Chapter 2. Generic vs. domain specific corpora to apply Natural Language Processing for Spanish Electronic Health Records

2.3. Generic vs. domain specific corpora for training models

$$
\begin{gathered}
\text { precision }=\frac{T P}{T P+F P} \\
\text { recall }=\frac{T P}{T P+F N} \\
F-\text { score }=\frac{2 \times \text { precision } \times \text { recall }}{\text { precision }+ \text { recall }} \\
\text { Accuracy }=\frac{T P+T N}{T P+F P+T N+F N}
\end{gathered}
$$

\subsubsection{General methodology followed in the training process}

A methodology to compare the performance of different corpora, generic and specific to the healthcare domain has been designed.

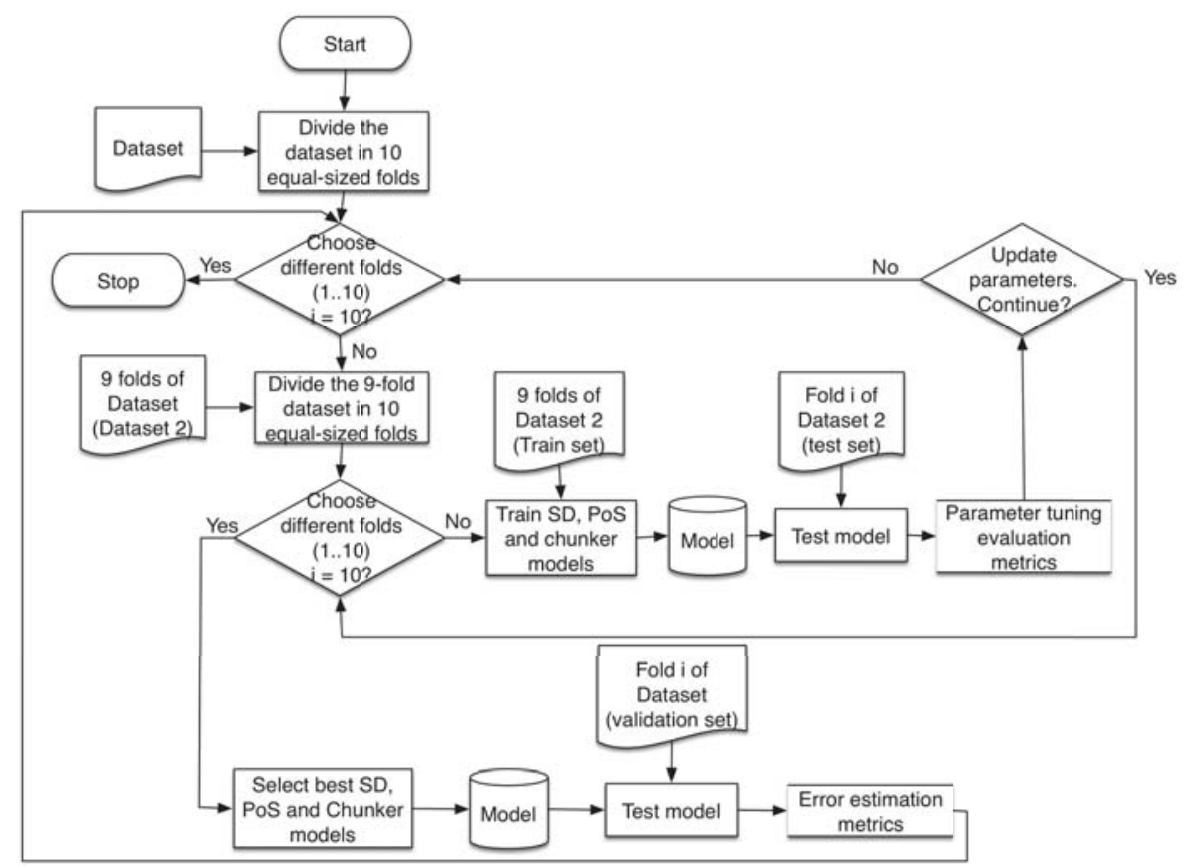

Figure 2.2: Flowchart describing the methodology used

In this scenario, a double nested 10-fold Cross-Validation (CV) methodology has been chosen. This methodology starts by dividing an initial dataset of documents (corpus) into 10 different equal-sized folds. Having nested 10 -folds, allows to generate a blind validation set from the first set of folds, which is used to measure the error in the training process, while the rest of the folds constitute a new dataset that is divided into another 10 folds in an inner loop, which is used following the 10 -fold $\mathrm{CV}$ for training a model with the given parameters (using 
Chapter 2. Generic vs. domain specific corpora to apply Natural Language Processing for Spanish Electronic Health Records 2.3. Generic vs. domain specific corpora for training models

9 of the folds), and using the missing as a test set to measure the performance of the model created. A flowchart describing this process can be seen in Figure 2.2.

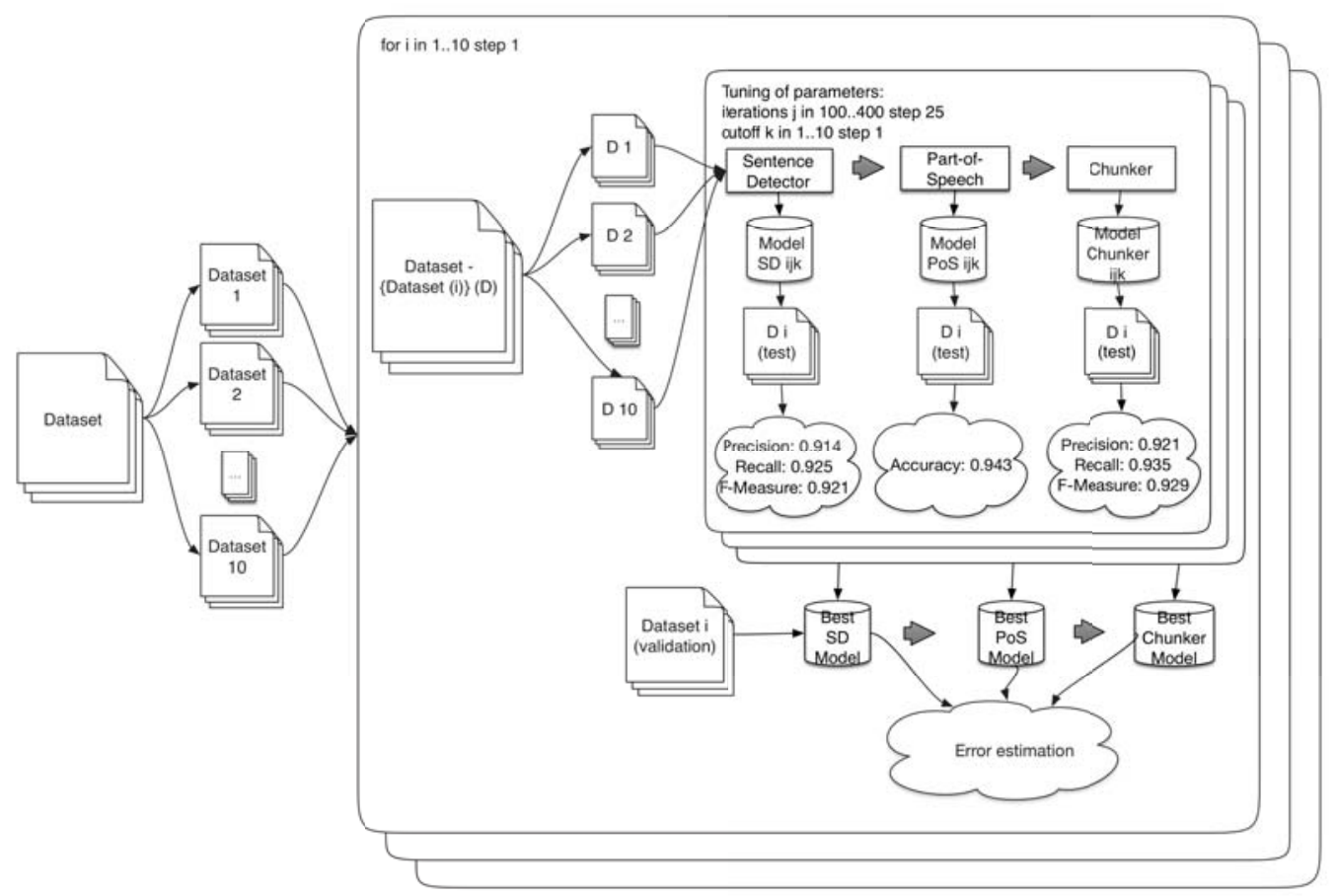

Figure 2.3: Description of the process followed to tune the parameters

The process followed in the execution of the experiments performed is depicted in Figure 2.3 and it is mainly based on the following steps:

1. Tuning of the parameters: In order to obtain the best performance from the trained models, a set of parameters must be tuned to obtain the appropriate values to maximize the performance for a given document set. These parameters are input values that are provided to the algorithms and they impact the performance of the trained models.

OpenNLP tools build different models depending on the value given to the expected parameters:

\# of iterations is a parameter which will tell how many iterations should be carried out in the training process. This parameter will be referenced as iters in Section 2.3.5.

cutoff refers to the number of times that a feature must appear in order to be considered in the model.

The type parameter is only needed for the training of Part-of-Speech models and it will determine the type of training. Its possible values are: maxent (Maximum entropy) or perceptron. The former has been used throughout the process as in [Buyko et al., 2006], [Savova et al., 2010].

2. Estimation of the error: In order to verify how well the different models are trained. 
Chapter 2. Generic vs. domain specific corpora to apply Natural Language Processing for Spanish Electronic Health Records

2.3. Generic vs. domain specific corpora for training models

\subsubsection{Datasets}

\section{Dataset D1}

This dataset has been extracted from generalist sources using already annotated corpora. Dataset D1 uses the Spanish version of AnCora.

This corpus is annotated at different levels: I) Lemma and Part-of-Speech, II) Syntactic constituents and functions, III) Argument structure and thematic roles, IV) Semantic classes of the verb, V) Denotative type of deverbal nouns, VI) Nouns related to WordNet synsets ${ }^{2}$, VII) Named Entities, VIII) Co-reference relations. AnCora corpus is mainly based on journalist texts covering different domains.

- Source: AnCora corpus

- Type of documents: General domain and newspapers

- \# of words: 547,212

- \# of sentences: 17,375

- \# of documents: 1,635

- Version: 3.0 es (Spanish version)

- Availability: Freely available at http://clic.ub.edu/corpus/ancora

- Original PoS tagset: EAGLES tagset

\section{Dataset D2}

The lack of annotated resources in the clinical domain in Spanish, specially those gathered from real data from patients, have led to the creation of Dataset D2.

D2 builds a gold standard dataset with linguistic annotations. This dataset is composed of 29 oncology documents containing 745 sentences and 10,241 words, from which 2,259 words are unique.

Three different Computer Science experts assisted by a Physician and a Computational Linguistic expert performed the annotation task on the generated corpus. A customized web application $^{3}$ was developed to aid the experts in the annotation process using the EAGLES tags [EAGLES Computational Lexicons Working Group, 1996].

An inter-annotator agreement (IAA) method similar to the one used in [Savova et al., 2010] was used to ensure the quality of the developed annotations. This method computes, in

\footnotetext{
${ }^{2}$ from the expression Syntactic sets

${ }^{3}$ This software has been made public and is available at https://github.com/rcostu/ EAGLES-Annotation
} 
Chapter 2. Generic vs. domain specific corpora to apply Natural Language Processing for Spanish Electronic

cases of disagreement in the annotation for a particular term, the most frequent annotation, from those assigned by the annotators.

- Source: EHR from a public hospital

- Type of documents: EHR and clinical reports

- \# of words: 10,241

- \# of sentences: 745

- \# of documents: 29

- Version: 1.0

- Availability: Proprietary due to confidentiality.

- PoS tagset: EAGLES tagset.

\section{Dataset D3}

Dataset D3 has been created as an extension of D2, gathering more documents creating a greater dataset. The documents in this dataset have also been automatically transformed to use two different PoS tagsets.

- Source: EHR from a public hospital

- Type of documents: EHR and clinical reports

- \# of words: 49,339

- \# of sentences: 4,117

- \# of documents: 150

- Version: 2.0

- Availability: Proprietary due to confidentiality.

- PoS tagset: EAGLES tagset (original annotation), Universal PoS tagset. 
Chapter 2. Generic vs. domain specific corpora to apply Natural Language Processing for Spanish Electronic Health Records

2.3. Generic vs. domain specific corpora for training models

\section{Dataset D4}

Dataset D4 is a generated subset from Dataset D1. This dataset is created to be an equal sized set as D3 in terms of the number of documents, which is relevant when training Sentence Detector and Part-of-Speech models.

Datasets $D 4_{1}, D 4_{2}, D 4_{3}, D 4_{4}, D 4_{5}, D 4_{6}, D 4_{7}, D 4_{8}, D 4_{9}, D 4_{10}, D 4_{11}$ and $D 4_{12}$

- Source: AnCora corpus

- Type of documents: General domain and newspapers

- \# of words: 38,153 - 50,024

- \# of sentences: 1,236 - 1,747

- \# of documents: 136 - 137

- Version: 3.0 es (Spanish version) subsets

- PoS tagset: EAGLES tagset (original annotation), Universal PoS tagset.

- Note: Each $D 4_{i}(i=1 . .12)$ is generated as a subset from the dataset D1 and the sizes vary from one to another but are roughly the same size as the dataset D3 used in terms of number of documents and are used to carry out the training of the Sentence Detector and the Part-of-Speech, whose training is based on the number of documents.

The complete description, in terms of number of words, sentences and documents for each $D 4_{i}$ ( $i$ from 1 to 12 ) is detailed in Table 2.1.

\begin{tabular}{|l|c|c|c|}
\hline & \# of words & \# of sentences & \# of documents \\
\hline$D 4_{1}$ & 42,558 & 1,418 & 136 \\
\hline$D 4_{2}$ & 41,169 & 1,399 & 137 \\
\hline$D 4_{3}$ & 44,974 & 1,400 & 137 \\
\hline$D 4_{4}$ & 45,826 & 1,479 & 137 \\
\hline$D 4_{5}$ & 47,840 & 1,573 & 136 \\
\hline$D 4_{6}$ & 44,200 & 1,453 & 136 \\
\hline$D 4_{7}$ & 38,153 & 1,236 & 136 \\
\hline$D 4_{8}$ & 42,776 & 1,379 & 136 \\
\hline$D 4_{9}$ & 44,251 & 1,433 & 136 \\
\hline$D 4_{10}$ & 45,704 & 1,470 & 136 \\
\hline$D 4_{11}$ & 50,024 & 1,747 & 136 \\
\hline$D 4_{12}$ & 42,364 & 1,359 & 136 \\
\hline
\end{tabular}

Table 2.1: Description of datasets $D 4_{i}$ ( $i$ from 1 to 12 ) 
Chapter 2. Generic vs. domain specific corpora to apply Natural Language Processing for Spanish Electronic

\section{Dataset D5}

Dataset D5 is similar to Dataset D4, as it is a subset generated from D1. This dataset is created to be an equal sized set as D3 in terms of the number of sentences, which is relevant in the training of Chunker models.

Datasets $D 5_{1}, D 5_{2}, D 5_{3}, D 5_{4}$ and $D 5_{5}$

- Source: AnCora corpus

- Type of documents: General domain and newspapers

- \# of words: 109,056

- \# of sentences: 3,469 - 3,470

- \# of documents: 327

- Version: 3.0 es (Spanish version) subsets

- PoS tagset: EAGLES tagset (original annotation), Universal PoS tagset.

- Note: Each $D 5_{i}(i=1 . .5)$ is generated as a subset from the dataset D1 and the sizes vary from one to another but are roughly the same size as the dataset D2 used in terms of number of sentences and are used to carry out the training of the Chunker, whose training is based on the number of sentences.

The complete description, in terms of number of words, sentences and documents for each $D 5_{i}$ ( $i$ from 1 to 5 ) is detailed in Table 2.2.

\begin{tabular}{|l|c|c|}
\hline & \# of words & \# of sentences \\
\hline$D 5_{1}$ & 109,057 & 3,469 \\
\hline$D 5_{2}$ & 108,962 & 3,470 \\
\hline$D 5_{3}$ & 108,836 & 3,469 \\
\hline$D 5_{4}$ & 110,419 & 3,469 \\
\hline$D 5_{5}$ & 109,904 & 3,469 \\
\hline
\end{tabular}

Table 2.2: Description of datasets $D 5_{j}$ ( $j$ from 1 to 5 )

\subsubsection{Experiments}

The experiments conducted follow the process explained in Section 2.3.3 in order to train models using Apache OpenNLP [OpenNLP, 2011] to extract information from texts written in Spanish, specially for the medical domain.

These experiments are usually carried out with values ranging from 100 to 350 iterations, with steps of 25 as in [Savova et al., 2010]. Values ranging from 100 to 400 with steps of 25 
Chapter 2. Generic vs. domain specific corpora to apply Natural Language Processing for Spanish Electronic Health Records

2.3. Generic vs. domain specific corpora for training models

have been chosen to broaden the training experiment. This is done so, because smaller steps have no such impact on reducing the final error. A lower number of iterations are typically not significant enough and higher iteration values lead to an increase in the computational time.

For the cutoff, typical values are taken from 1 to 10 [Savova et al., 2010], and our experiments follow this same distribution. In this case, lower values are nonsense because a word has to appear in order to be taken into account. Higher values usually mean the loss of relevant information that is not repeated very often in the corpus.

The possible combinations of the first two parameters lead to the generation of different models.

In the incoming subsections the experiments performed to train the models are explained.

\section{Experiment 1}

The main goal behind the experiment is to detect how models behave and to be able to analyze whether domain specific annotated corpus (D2) help to create new models outperforming or not models generated with generic corpora (D1).

The process developed to perform the Experiment 1 is composed of the following phases:

1. Datasets D1 and D2 are cleansed to remove irrelevant metadata and solve encoding problems, and prepared to the proper task, according to OpenNLP standards:

- For the Sentence Detector, documents in D1 and D2 are joined in a single document for each dataset so each line of this document corresponds to a complete sentence, and a blank line separates each of the documents in the dataset.

- For the Part-of-Speech, the same approach is followed, but in this case all tokens are white-space separated from each other in each sentence, and attached a tag with an underscore (example: La_DET cabeza_NOUN mostraba_VERB una_DET contusión_NOUN).

- In the case of the Chunker, each line of the final document corresponds to a single token, followed by the PoS assigned, and finally followed by the tag corresponding to the phrase the token belongs to.

2. The newly generated documents for D1 and D2, are split into training and evaluation datasets for the experiment. The validation of all the models is done on the basis of a 10-fold CV with 80/20 split as in [Savova et al., 2010] where $80 \%$ is used for training and $20 \%$ for testing. Experiments were conducted splitting the training dataset into ten folds of an equivalent number of documents inside each fold.

3. After training the models, they have been tested with particular values for the parameters as explained in what follows. 
Chapter 2. Generic vs. domain specific corpora to apply Natural Language Processing for Spanish Electronic Health Records 2.3. Generic vs. domain specific corpora for training models

Note that OpenNLP's Tokenizer is not trained. For Dataset D1, tokens are already provided within the corpus, so tokenization is not needed. In the case of Dataset D2, a custom tokenizer has been build in order to extract the tokens in the medical texts to annotate, in which punctuation symbols, multiple spaces and line breaks to determine the tokens, as well as the particularities of this domain such as doses or other metrics have been taken into account.

Figure 2.4 depicts the overall process developed in order to conduct Experiment 1.

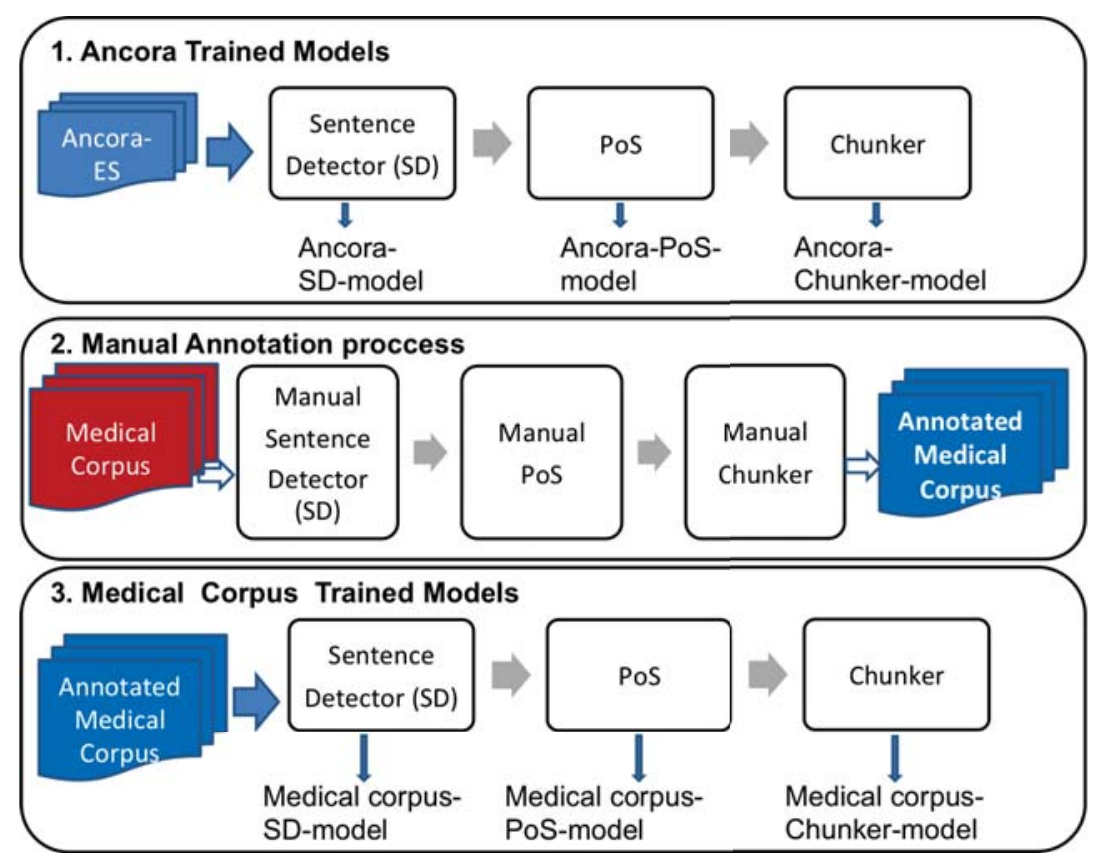

Figure 2.4: Processes followed to train the models from a generic and a domain specific corpus

\section{Sentence Detector}

The creation of Sentence Detector models is performed with iteration values ranging from 100 to 600 and cut-off values from 1 to 16 for each fold. The optimal values resulted to be 600 iterations and a cut-off of 16 for D1, and 475 iterations and a cut-off of 7 for D2 (these corresponds to the values of iterations and cut-off to which models generated have in average the highest F-Measure).

For these values, the best models obtained performed for D1 with a precision of 0.948 , a recall of 0.963 and a F-Measure of 0.955 . For D2 the performance values obtained are precision of 0.827 , a recall of 0.714 and a F-Measure of 0.766 .

The main source of errors in this particular component was due to headings or sentences that did not use proper punctuation symbols, so the models were not able to reproduce the sentences correctly.

\section{PoS tagger}

Experimentation with iteration values ranging from 100 to 400 and cut-off values from 1 to 10 for each corpus has been made. The optimal values were 100 iterations and a cut-off of 2 for D1, and 100 iterations and a cut-off of 1 for D2. 
Chapter 2. Generic vs. domain specific corpora to apply Natural Language Processing for Spanish Electronic Health Records

2.3. Generic vs. domain specific corpora for training models

It is worth noting that D2 includes great amounts of abbreviations and short sentences, so the cut-off value is intended to be low.

Models generated from D1 showed an accuracy of 0.921 while those generated from D2 got an accuracy of 0.774 .

\section{Chunker}

For the creation of Chunker models, training with iteration values ranging from 100 to 400 and cut-off values from 1 to 10 has been performed. The optimal values were 100 iterations and a cut-off of 3 for D1, and 150 iterations and a cut-off of 3 for D2.

Precision, recall an F-Measure for D1 models were 0.908, 0.907, and 0.907 respectively. D2 models performed with a precision of 0.852 , a recall of 0.860 , and a F-Measure of 0.856 .

\section{Comparison of the models}

Once the models have been generated, both using D1 and D2, a comparison of the results is made (see Table 2.3). The results shown in the table are the averaged results obtained from the training process.

\begin{tabular}{|l|c|c|}
\hline & D1 & D2 \\
\hline Sentence Detector & & \\
Precision & 0.948 & 0.828 \\
Recall & 0.963 & 0.714 \\
F-Measure & 0.955 & 0.766 \\
Part of Speech & & \\
Accuracy & 0.921 & 0.774 \\
Chunker & & \\
Precision & 0.908 & 0.852 \\
Recall & 0.907 & 0.860 \\
F-Measure & 0.907 & 0.856 \\
\hline
\end{tabular}

Table 2.3: General precision, recall, F-measure and accuracy values obtained in the training process

Trained models are intended to be applied in a medical environment. This way, the comparison is done with the following process: i) From the ten models generated for the best iterations and cut-off values for each dataset in each component (Sentence Detector, PoS, and Chunker), the one with the best F-Measure and accuracy is the one selected to evaluate; ii) The medical texts that have not been used in the training of D2 selected model for each component are the ones used to evaluate both the AnCora and in-house corpus; iii) Results are gathered from the evaluation and can be observed in the table 2.4.

Once the results have been analyzed, it can be seen that the training on the Sentence Detector is the same when the maximal values are obtained and the best model for those values is selected for evaluation, so the differences in the corpora are not significant for the delimitation of the phrases in a general corpus or a medical one for Spanish texts. The results for both corpora have obtained a precision of 0.904 , a recall of 0.842 and a F-Measure of 0.872 . 


\begin{tabular}{|l|c|c|}
\hline & D1 & D2 \\
\hline Sentence Detector & & \\
Precision & 0.904 & 0.904 \\
Recall & 0.842 & 0.842 \\
F-Measure & 0.872 & 0.872 \\
Part of Speech & & \\
Accuracy & 0.946 & 0.836 \\
Chunker & & \\
Precision & 0.904 & 0.891 \\
Recall & 0.914 & 0.894 \\
F-Measure & 0.909 & 0.893 \\
\hline
\end{tabular}

Table 2.4: Values comparison when evaluating the models with medical texts

However, performance is more important when analyzing the Part of Speech and the Chunker, because of all the medical concepts and the huge differences in the way physicians write texts. D1 models outperforms D2 models on the Part of Speech, while both performed similarly in the Chunker. Accuracy for the D1 in the Part of Speech was 0.946, while D2 models performed 0.836. In the Chunker evaluation, the results are similar: D1 models showed a precision of 0.904 , a recall of 0.914 and a F-Measure of 0.909 . D2 models performed with a precision of 0.891 , a recall of 0.894 and a F-measure of 0.893 for the same component.

\section{Experiment 2}

This experiment has two main objectives: I) To check the influence on the quality of the models of domain-independent corpora vs. the domain-dependent ones using bigger datasets than experiment 1; II) to analyze the performance and influence of the amount of semantic information of the different tagset systems in the final model produced.

The learning process is set out as pseudocode in Listing 2.1 and explained in detail to clarify the process behind the training and validation of the different models.

From lines 1 to 9 , the outer CV used to carry out the validation of the models is explained. As can be observed in line 2, the dataset $\mathrm{D}$ used as an input for the training and validation is divided into 10 same-sized folds $\left(D_{1}^{\prime}\right.$ to $\left.D_{10}^{\prime}\right)$. Then, in line 4, the ten iterations to perform a tenfold $\mathrm{CV}$ are depicted. In each iteration a new dataset $D_{\text {trainingInner }}$ is created by joining all the D' datasets except the $D_{i}^{\prime}$ that will be used for validation (line 5). Line 6 shows the call of the procedure that will carry out the tuning of the parameters with this new dataset $D_{\text {trainingInner }}$ and will generate the model M. Finally, this procedure will validate the model $\mathrm{M}$ generated in the last step with the fold $D_{i}^{\prime}$ corresponding to this iteration.

From lines 11 to 26 the inner CV used to carry out the tuning of the parameters for the generation of the models is explained. This procedure receives an input parameter which is the dataset $\mathrm{D}$ to be used and produces as an output parameter $\mathrm{M}$ the model. 
Chapter 2. Generic vs. domain specific corpora to apply Natural Language Processing for Spanish Electronic Health Records

2.3. Generic vs. domain specific corpora for training models

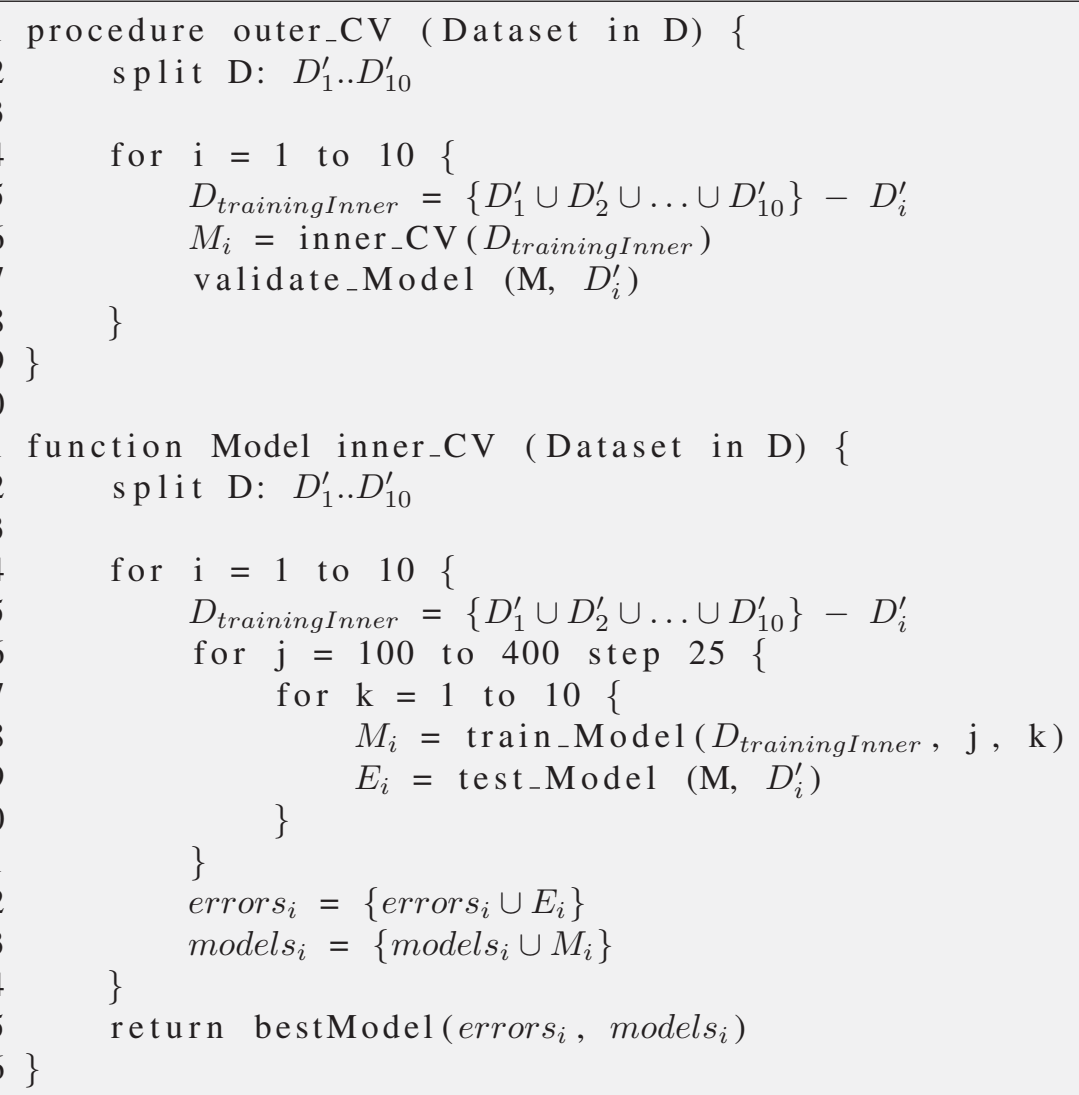

Listing 2.1: Pseudocode of the cross-validation

First, in line 12, the Dataset is divided into 10 same-sized folds ( $D_{1}^{\prime}$ to $D_{10}^{\prime}$ ). From line 14 to line 22, the iterations for the ten-fold CV are carried out. In each iteration, as can be seen in line 15 , a new dataset $D_{\text {trainingInner }}$ is created from the folds D' except the $D_{i}^{\prime}$ corresponding to the iteration that will be used as a test set. Line 16 shows the loop for the iterations parameter, from 100 to 400 in steps of 25 . The next line shows the loop for the cut-off parameter, counting from 1 to 10 . A model is trained within both loops with the dataset $D_{\text {trainingInner }}$ created and the corresponding iterations and cut-off parameters, it is then tested against the dataset $D_{i}^{\prime}$.

Note that the training is carried out with a given combination for the iterations and cut-off values and it is then tested to obtain the precision, recall, F-measure and accuracy statistical values. All the iterations and cut-off combinations build a new model which is tested so finally end up with 130 different models in each call to the inner_CV procedure created. As this procedure is called 10 times for the outer procedure outer_CV, a total of 1,300 models are trained and tested.

Each of these steps is carried out using a nested ten-fold CV procedure where the datasets are divided into ten folds for each iteration, 9 folds are for training the corresponding models and one for testing the trained models. This nested CV can be found in Figure 2.5 and Listing 2.1.

Nested CV has to be carried out in order to obtain unbiased results. As is well known, Cross-Validation methods are proven to be unbiased only if all the aspects of classifier training 
Chapter 2. Generic vs. domain specific corpora to apply Natural Language Processing for Spanish Electronic Health Records

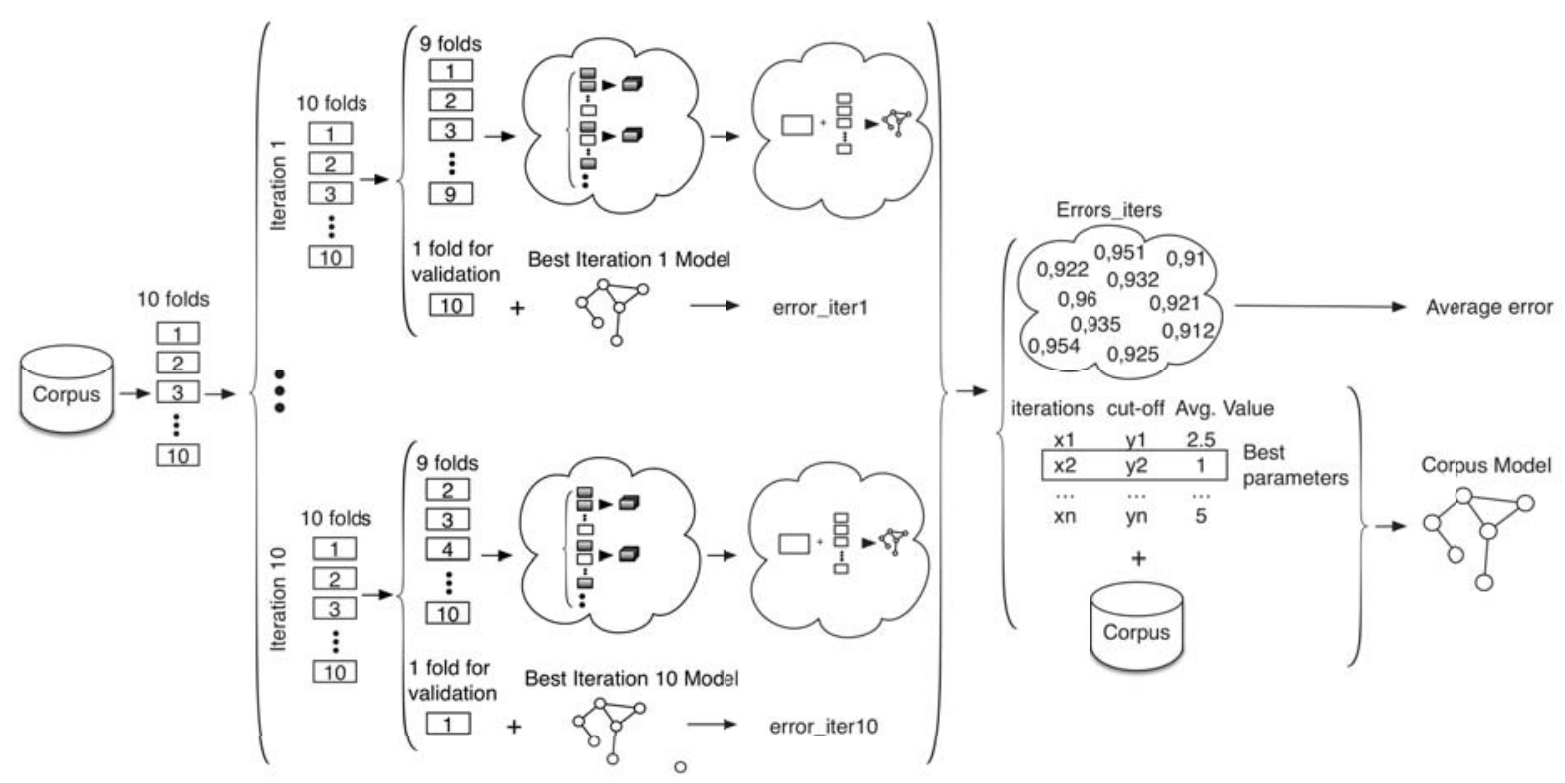

Cross Validation

Figure 2.5: Definition of the complete process with nested CV



Figure 2.6: Definition of the inner CV to tune the parameters 
Chapter 2. Generic vs. domain specific corpora to apply Natural Language Processing for Spanish Electronic Health Records

2.3. Generic vs. domain specific corpora for training models

(feature selection, parameter tuning, etc.) take place within the CV loop [Varma and Simon, 2006]. Thus, it is necessary to carry out an inner CV per iteration (see Figure 2.6) of the outer CV (see Figure 2.7) to tune the parameter values of both corpora models. This procedure provides a reliable estimation of the error when there are not enough instances in the dataset [Iizuka et al., 2003].

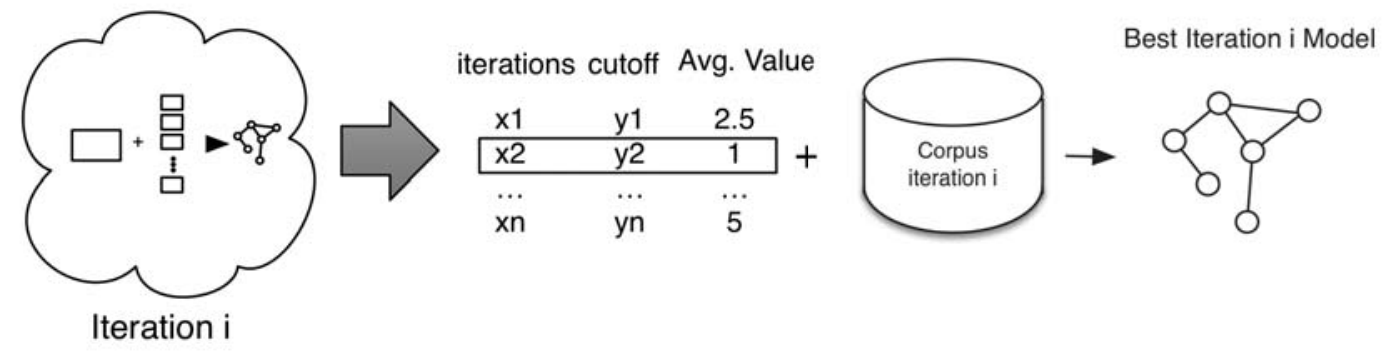

Figure 2.7: Definition of the outer CV to build and validate the models

In order to achieve the objectives set for Experiment 2, this has been divided into 3 subexperiments, which are explained in the following subsections.

\section{Experiment 2.1}

The goal of this sub-experiment is to test the relationship between the structure of the documents used and the performance on the Sentence Detector models.

\begin{tabular}{|c|c|c|c|}
\hline & \multicolumn{3}{|c|}{ Sentence Detector } \\
\hline Dataset & iters & cut-off & F-Measure \\
\hline $\mathrm{D} 1$ & 375 & 10 & 0.958 \\
$\mathrm{D} 3$ & 125 & 4 & 0.725 \\
$D 4_{1}$ & 300 & 9 & 0.959 \\
$D 4_{2}$ & 250 & 6 & 0.957 \\
$D 4_{3}$ & 350 & 4 & 0.962 \\
$D 4_{4}$ & 275 & 7 & 0.957 \\
$D 4_{5}$ & 275 & 8 & 0.948 \\
$D 4_{6}$ & 275 & 9 & 0.958 \\
$D 4_{7}$ & 100 & 1 & 0.962 \\
$D 4_{8}$ & 100 & 10 & 0.946 \\
$D 4_{9}$ & 100 & 10 & 0.942 \\
$D 4_{10}$ & 375 & 6 & 0.936 \\
$D 4_{11}$ & 200 & 4 & 0.965 \\
$D 4_{12}$ & 200 & 4 & 0.954 \\
\hline
\end{tabular}

Table 2.5: Experiment 2.1 - Sentence Detector parameter tuning for the different datasets

In this experiment, the goal is to check whether the structure followed in different documents leads to better sentence detection. Note that dealing with medical texts in dataset D3, the 
Chapter 2. Generic vs. domain specific corpora to apply Natural Language Processing for Spanish Electronic Health Records 2.3. Generic vs. domain specific corpora for training models

structure is not so well defined as different doctors typically write without following a common pattern. This experiment should lead to a better performance using D1 and $D 4_{i}$ ( $i$ from 1 to 12 ) datasets, as the generic corpus source are typically newspapers, where language structures are preserved.

In order to carry out this experiment, datasets D1, D3 and $D 4_{i}$ ( $i$ from 1 to 12 ) have been used.

The parameters have been tuned using the inner CV with the training and test sets from datasets D1, D3 and D4 respectively. The parameters obtained that resulted in the maximal values for the average F-Measures presented in the experimentation are detailed in Table 2.5. It can be seen that models generated with D3 underperform those obtained with the other datasets.

Table 2.6 details the results of the validation carried out for the datasets.

\begin{tabular}{|c|c|c|c|}
\hline & \multicolumn{3}{|c|}{ Sentence Detector } \\
\hline Dataset & Precision & Recall & F-Measure \\
\hline $\mathrm{D} 1$ & 0.947 & 0.964 & 0.955 \\
$\mathrm{D} 3$ & 0.798 & 0.645 & 0.713 \\
$D 4_{1}$ & 0.948 & 0.970 & 0.959 \\
$D 4_{2}$ & 0.953 & 0.969 & 0.961 \\
$D 4_{3}$ & 0.946 & 0.961 & 0.954 \\
$D 4_{4}$ & 0.940 & 0.949 & 0.944 \\
$D 4_{5}$ & 0.945 & 0.963 & 0.954 \\
$D 4_{6}$ & $\mathbf{0 . 9 5 6}$ & $\mathbf{0 . 9 7 5}$ & $\mathbf{0 . 9 6 5}$ \\
$D 4_{7}$ & 0.943 & 0.960 & 0.951 \\
$D 4_{8}$ & 0.925 & 0.961 & 0.943 \\
$D 4_{9}$ & 0.926 & 0.952 & 0.939 \\
$D 4_{10}$ & 0.949 & 0.955 & 0.952 \\
$D 4_{11}$ & 0.939 & 0.955 & 0.947 \\
$D 4_{12}$ & 0.934 & 0.952 & 0.943 \\
\hline
\end{tabular}

Table 2.6: Experiment 2.1 - Sentence Detector: precision, recall and F-measures for the different datasets

As dataset D3 contains actual medical texts, the usual grammatical structures are not followed as in datasets D1, $D 4_{i}$ ( $i$ from 1 to 12 ). Consequently this is reflected in the results as the Sentence Detector models from D3 have lower F-Measures than those produced from D1 and $D 4_{i}$ ( $i$ from 1 to 12 ).

\section{Experiment 2.2}

Experiment 2.2 tries to verify whether the Part-of-Speech and Chunker domain-dependent models outperform generic corpora models.

To prevent the size of the dataset from biasing the results $D 4_{i}$ ( $i$ from 1 to 12 ) instead of D1 has been used. This bias due to the size in tagged models can be seen in Experiment 1, where bigger sized corpora - having more tagged information - led to better results in the 
Chapter 2. Generic vs. domain specific corpora to apply Natural Language Processing for Spanish Electronic Health Records

2.3. Generic vs. domain specific corpora for training models

created models.

Domain-dependent models should obtain better results when analyzing the Part-of-Speech than models trained with generic corpora, when the document set is somewhat similar in size.

The vocabulary and the way of writing in a particular domain should be represented into the trained models, which in the testing and validation process should show fewer errors than those obtained with the models trained with generic documents.

For this purpose, datasets D3 and $D 4_{i}$ ( $i$ from 1 to 12 ) have been used in the training of the Part-of-Speech models, and datasets D3 and $D 5_{j}$ ( $j$ from 1 to 5) have been used in the training of the Chunker models. These datasets have been selected for this experiment in order to compare equally-sized corpora in terms of number of documents.

\begin{tabular}{|c|c|c|c|c|c|c|}
\hline & \multicolumn{3}{|c|}{ Part-of-Speech (EAGLES) } & \multicolumn{2}{c|}{ Part-of-Speech (Universal) } \\
\hline Dataset & iters & cut-off & Accuracy & iters & cut-off & Accuracy \\
\hline $\mathrm{D} 3$ & 350 & 1 & 0.931 & 400 & 1 & 0.973 \\
$D 4_{1}$ & 100 & 2 & 0.878 & 100 & 2 & 0.938 \\
$D 4_{2}$ & 100 & 2 & 0.882 & 175 & 2 & 0.939 \\
$D 4_{3}$ & 100 & 3 & 0.886 & 125 & 3 & 0.940 \\
$D 4_{4}$ & 100 & 2 & 0.881 & 125 & 2 & 0.940 \\
$D 4_{5}$ & 100 & 2 & 0.883 & 100 & 3 & 0.939 \\
$D 4_{6}$ & 100 & 2 & 0.886 & 100 & 2 & 0.942 \\
$D 4_{7}$ & 125 & 1 & 0.877 & 125 & 1 & 0.936 \\
$D 4_{8}$ & 125 & 2 & 0.880 & 125 & 2 & 0.938 \\
$D 4_{9}$ & 375 & 1 & 0.885 & 125 & 2 & 0.940 \\
$D 4_{10}$ & 100 & 2 & 0.888 & 200 & 2 & 0.943 \\
$D 4_{11}$ & 125 & 1 & 0.889 & 200 & 2 & 0.942 \\
$D 4_{12}$ & 100 & 1 & 0.878 & 100 & 2 & 0.941 \\
\hline
\end{tabular}

Table 2.7: Experiment 2.2 - Parameter tuning with best average accuracies for the Part-of-Speech using both tagsets

The training of the datasets has been carried out and the parameters obtained together with their average accuracies can be seen in Table 2.7 for the Part-of-Speech models. This training has been performed separately using EAGLES and Universal Part-of-Speech tagsets.

The parameters obtained generate models whose accuracies are higher for those models corresponding to dataset D3 than in any of the models corresponding to datasets $D 4_{i}$ ( $i$ from 1 to 12 ). The final validation is shown in Table 2.8 .

Table 2.9 shows the parameters obtained and the corresponding F-Measures for the Chunker Models. Note that although Chunker tagset is unique, the results are divided in two different columns, as OpenNLP uses also the information gathered from the Part-of-Speech annotation and both PoS tagsets have been used. In this case the results obtained in the models trained with dataset D3 outperform those trained with datasets $D 5_{j}$ ( $j$ from 1 to 5 ). 
Chapter 2. Generic vs. domain specific corpora to apply Natural Language Processing for Spanish Electronic Health Records 2.3. Generic vs. domain specific corpora for training models

\begin{tabular}{|c|c|c|}
\hline & Part-of-Speech (EAGLES) & Part-of-Speech (Universal) \\
\hline Dataset & Accuracy & Accuracy \\
\hline $\mathrm{D} 3$ & $\mathbf{0 . 9 3 3}$ & $\mathbf{0 . 9 7 5}$ \\
$D 4_{1}$ & 0.886 & 0.939 \\
$D 4_{2}$ & 0.886 & 0.941 \\
$D 4_{3}$ & 0.885 & 0.942 \\
$D 4_{4}$ & 0.888 & 0.941 \\
$D 4_{5}$ & 0.888 & 0.942 \\
$D 4_{6}$ & 0.891 & 0.944 \\
$D 4_{7}$ & 0.880 & 0.939 \\
$D 4_{8}$ & 0.888 & 0.940 \\
$D 4_{9}$ & 0.890 & 0.943 \\
$D 4_{10}$ & 0.887 & 0.939 \\
$D 4_{11}$ & 0.887 & 0.944 \\
$D 4_{12}$ & 0.884 & 0.939 \\
\hline
\end{tabular}

Table 2.8: Experiment 2.2 - Accuracies obtained for the Part-of-Speech using both tagsets in D3 and $D 4_{i}$ ( $i$ from 1 to 12 )

\begin{tabular}{|c|c|c|c|c|c|c|}
\hline & \multicolumn{3}{|c|}{ Chunker (EAGLES) } & \multicolumn{3}{c|}{ Chunker (Universal) } \\
\hline Dataset & iters & cut-off & F-Measure & iters & cut-off & F-Measure \\
\hline D3 & 375 & 5 & 0.976 & 400 & 10 & 0.996 \\
$D 5_{1}$ & 125 & 2 & 0.914 & 100 & 8 & 0.902 \\
$D 5_{2}$ & 125 & 2 & 0.911 & 175 & 6 & 0.902 \\
$D 5_{3}$ & 100 & 2 & 0.910 & 125 & 4 & 0.902 \\
$D 5_{4}$ & 100 & 2 & 0.913 & 125 & 4 & 0.901 \\
$D 5_{5}$ & 100 & 3 & 0.912 & 100 & 9 & 0.902 \\
\hline
\end{tabular}

Table 2.9: Experiment 2.2 - Parameter tuning with best average F-Measures for the Chunker using both Part-of-Speech tagsets 
Chapter 2. Generic vs. domain specific corpora to apply Natural Language Processing for Spanish Electronic Health Records

2.3. Generic vs. domain specific corpora for training models

Finally, Table 2.10 summarizes the results obtained in the validation process for the Chunker models.

\begin{tabular}{|c|c|c|c|c|c|c|}
\hline & \multicolumn{3}{|c|}{ Chunker (EAGLE) } & \multicolumn{3}{c|}{ Chunker (Universal) } \\
\hline Dataset & Precision & Recall & F-Measure & Precision & Recall & F-Measure \\
\hline D3 & $\mathbf{0 . 9 7 4}$ & $\mathbf{0 . 9 7 6}$ & $\mathbf{0 . 9 7 5}$ & $\mathbf{0 . 9 9 6}$ & $\mathbf{0 . 9 9 5}$ & $\mathbf{0 . 9 9 6}$ \\
$D 5_{1}$ & 0.918 & 0.912 & 0.915 & 0.904 & 0.899 & 0.901 \\
$D 5_{2}$ & 0.915 & 0.911 & 0.913 & 0.902 & 0.901 & 0.901 \\
$D 5_{3}$ & 0.917 & 0.910 & 0.913 & 0.903 & 0.899 & 0.901 \\
$D 5_{4}$ & 0.918 & 0.912 & 0.915 & 0.905 & 0.900 & 0.902 \\
$D 5_{5}$ & 0.917 & 0.914 & 0.916 & 0.905 & 0.903 & 0.904 \\
\hline
\end{tabular}

Table 2.10: Experiment 2.2 - Parameters with best average F-Measures for the Chunker using both Part-of-Speech tagsets

These results show that models trained with dataset D3 outperform those trained with datasets $D 5_{j}$ ( $j$ from 1 to 5). Therefore, this experiment confirms that dealing with equal-sized corpus, models generated from domain-dependent corpora outperform models generated from the generic ones.

As part of this experimentation another important fact can be observed: The use of the Universal PoS tagset increases the accuracies obtained leading to better overall results $(+4-5 \%)$ in the analysis of the Part-of-Speech. These results show that those tagsets with fewer semantical information outperform the results obtained with the EAGLES tagset when analyzing the Part-of-Speech.

It is important to note that the use of Universal PoS tags in the training of the Chunker models leads to lower values obtained in the datasets $D 5_{i}$ ( $j$ from 1 to 5). The generic nature of the dataset can be at the root of the decrease in performance. However, note that PoS depends on the annotation process and in order to validate this behavior, further experiments should be carried out with different generic corpora.

\section{Experiment 2.3}

In this sub-experiment the goal is to test whether a huge amount of generic information can be used in order to train models that could have similar results to those obtained with a smaller-sized domain-dependent corpus. This is to check whether the size of the corpus can be determinant in a better quality of the final models. In fact, a comparison of the performance of a generic corpus vs. a smaller domain-dependent one has been made.

This premise is sustained by the huge amount of information which, indeed, should lead to a better training of the models, and even compete with the models trained with the specifics of the domain. In this case, datasets D1 and D3 have been used.

Experiments 2.2 and 2.3 should show the difference in the semantic information provided for the EAGLES and Universal PoS tagsets. While the EAGLES tagset is richer as it gives more semantic information for any given tag, which is very good for a rich language such as 
Chapter 2. Generic vs. domain specific corpora to apply Natural Language Processing for Spanish Electronic Health Records 2.3. Generic vs. domain specific corpora for training models

Spanish, the Universal PoS tagset gives less semantic information, leading to a simpler annotation. This difference in the semantic information provided by the tagsets should lead to a better performance for the Universal PoS tagset, as the number of tags and the information provided is poorer, which should give rise to better-trained models.

In Table 2.11 the parameters obtained and the associated Accuracy obtained in the Partof-Speech can be observed.

\begin{tabular}{|c|c|c|c|c|c|c|}
\hline & \multicolumn{2}{|c|}{ Part-of-Speech (EAGLES) } & \multicolumn{3}{c|}{ Part-of-Speech (Universal) } \\
\hline Dataset & iters & cut-off & Accuracy & iters & cut-off & Accuracy \\
\hline D1 & 100 & 2 & 0.926 & 200 & 2 & 0.965 \\
D3 & 350 & 1 & 0.933 & 400 & 1 & 0.974 \\
\hline
\end{tabular}

Table 2.11: Experiment 2.3 - Parameter tuning with best average accuracies for the Part-of-Speech using both tagsets

Table 2.12 shows the Accuracy obtained in both datasets D1 and D3 in the validation process with an previously unseen set of documents.

\begin{tabular}{|c|c|c|}
\hline & Part-of-Speech (EAGLES) & Part-of-Speech (Universal) \\
\hline Dataset & Accuracy & Accuracy \\
\hline D1 & 0.927 & 0.966 \\
D3 & $\mathbf{0 . 9 3 3}$ & $\mathbf{0 . 9 7 5}$ \\
\hline
\end{tabular}

Table 2.12: Experiment 2.3 - Accuracies obtained for the Part-of-Speech using both PoS tagsets in D1 and D3

The results presented for the Part-of-Speech show that the accuracy is higher for dataset D3 than those in dataset D1. It should also be noted that the accuracy is also higher when using Universal PoS tags.

Table 2.13 summarizes the parameters obtained and the associated F-measure obtained in the Chunker models, while Table 2.14 shows the precision, recall and F-measure obtained in the validation of the Chunker models.

\begin{tabular}{|c|c|c|c|c|c|c|}
\hline & \multicolumn{3}{|c|}{ Chunker (EAGLES) } & \multicolumn{3}{c|}{ Chunker (Universal) } \\
\hline Dataset & iters & cut-off & F-Measure & iters & cut-off & F-Measure \\
\hline D1 & 100 & 2 & 0.933 & 100 & 2 & 0.917 \\
D3 & 375 & 5 & 0.976 & 400 & 10 & 0.996 \\
\hline
\end{tabular}

Table 2.13: Experiment 2.3 - Parameters with best average F-Measures for the Chunker using both Part-of-Speech tagsets in D1 and D3

As can be seen, the results for the Chunker show that the models trained with dataset D3 outperform those trained with dataset D1. The use of domain-specific corpora outperforms generic ones but the differences between the different results show that the performance can be 
Chapter 2. Generic vs. domain specific corpora to apply Natural Language Processing for Spanish Electronic Health Records

2.3. Generic vs. domain specific corpora for training models

close enough to be useful in applying generic corpora trained models in a specific field. This can be of use in those cases where neither time nor annotated corpora are available.

\begin{tabular}{|c|c|c|c|}
\hline & \multicolumn{3}{|c|}{ Chunker (EAGLES) } \\
\hline Dataset & Precision & Recall & F-Measure \\
\hline D1 & 0.934 & 0.929 & 0.931 \\
D3 & $\mathbf{0 . 9 7 4}$ & $\mathbf{0 . 9 7 6}$ & $\mathbf{0 . 9 7 5}$ \\
\hline & \multicolumn{3}{|c|}{ Chunker (Universal) } \\
\hline Dataset & Precision & Recall & F-Measure \\
\hline D1 & 0.917 & 0.914 & 0.916 \\
D3 & $\mathbf{0 . 9 9 6}$ & $\mathbf{0 . 9 9 5}$ & $\mathbf{0 . 9 9 6}$ \\
\hline
\end{tabular}

Table 2.14: Experiment 2.3 - F-measures obtained for the Chunker using both Part-of-Speech tagsets in D1 and D3

\section{Final remarks on Experiment 2}

By these means, a comparison of the different performances in the learning process, when using different sizes and types of documents in the corpora used, can be made, specifically when applying NLP techniques to the medical domain. The results for these models are shown in Table 2.15.

\begin{tabular}{|l|c|c|c|c|}
\hline Datasets & D1 & D3 & D4 & D5 \\
\hline Sentence Detector & & & & \\
Precision & $\mathbf{0 . 9 4 7}$ & 0.798 & 0.942 & \\
Recall & $\mathbf{0 . 9 6 4}$ & 0.645 & 0.960 & \\
F-Measure & $\mathbf{0 . 9 5 5}$ & 0.713 & 0.951 & \\
Part-of-Speech (EAGLES) & & & & \\
Accuracy & 0.927 & $\mathbf{0 . 9 3 3}$ & 0.887 & \\
Part-of-Speech (Universal) & & & & \\
Accuracy & 0.966 & $\mathbf{0 . 9 7 5}$ & 0.941 & \\
Chunker (EAGLES) & & & & \\
Precision & 0.934 & $\mathbf{0 . 9 7 4}$ & & 0.917 \\
Recall & 0.929 & $\mathbf{0 . 9 7 6}$ & & 0.912 \\
F-Measure & 0.931 & $\mathbf{0 . 9 7 5}$ & & 0.914 \\
Chunker (Universal) & & & & \\
Precision & 0.917 & $\mathbf{0 . 9 9 6}$ & & 0.904 \\
Recall & 0.914 & $\mathbf{0 . 9 9 5}$ & & 0.900 \\
F-Measure & 0.916 & $\mathbf{0 . 9 9 6}$ & & 0.902 \\
\hline
\end{tabular}

Table 2.15: General Precision, Recall, F-Measure and Accuracy values obtained

However, even though these differences exist, the analysis carried out on the results for each given corpus validated with their own dataset, show that the in-house corpus outperforms 
the AnCora corpus, which gives us an indication that domain-driven corpora should outperform those of a non-specific domain, even though, generic corpora compiled from a greater number of documents and sentences can be used in specific domains.

\subsection{Discussion}

The lack of domain specific corpora for the healthcare domain to train NLP models and the cost associated with the efforts to create these resources have led to the analysis performed. In this analysis, the improvement on the use of these domain specific corpora against existing generic corpora has been studied.

The analysis performed shows that:

- Generic corpora applied to a blind test set of medical documents clearly outperforms domain specific corpora when the former is much larger.

- Generic corpora is able to detect better sentences even when the size of the corpus is equal to the domain specific one.

- Domain specific corpora trained models outperform generic corpora on the tasks of PoS tagging and Chunker tagging when using EAGLES and Universal PoS tagsets with equallysized corpora.

- Domain specific corpora trained models outperform the ones generated with generic corpora on the tasks of PoS tagging and Chunker tagging with both tagsets, being the generic corpora much larger than the domain specific one. However, the differences in PoS tagging (with a significance $<0.01$ ), show that a generic corpora can be used to train models and applied in the healthcare domain.

Therefore, the costs of creating domain specific annotated corpora can be reduced by using large amounts of annotated documents in generic corpora to train models to be used in the medical domain.

Furthermore, as a result of this analysis, two added-valued products have been created: I) An in-house annotated corpus gathered from reports included in EHRs; and II) a customized tool to annotate corpus using the EAGLES PoS tagset.

The analysis done, the creation of this new corpus and the experiments performed have led to the achievement of Objective O1.

The results of the research performed have produced the following publication:

- Roberto Costumero, Ángel García-Pedrero, Consuelo Gonzalo-Martín, Ernestina Menasalvas, and Socorro Millan. Text analysis and information extraction from Spanish written documents. In International Conference on Brain Informatics and Health, pages 188-197. Springer International Publishing, 2014. DOI: 10.1007/978-3-319-09891-3_18 


\section{CHAPTER 3}

\section{Addressing specific challenges in the application of Natural Language Processing for Spanish Electronic Health Records}

\subsection{Introduction}

Texts in the medical domain present particular characteristics differentiating them from generic texts. EHRs contain dates, metrics to define drug doses, complex entities (regarding parts of the body, diseases, etc.), negated terms, acronyms, or medical scales to determine patient severity and other conditions.

Currently there are no methods to extract this kind of information from Spanish written texts in EHRs. Therefore, the main objective of this chapter is to analyze the challenges affecting these texts and give a solution for them. In particular, this thesis addresses the following problems:

1. Detection of negated terms, as many of the entities mentioned in medical texts appear in negated form, and no solution had been proposed for Spanish.

2. Detection of different medical entities such as diseases, symptoms or drug names, which constitute the most common source of information from EHRs.

3. Detection and disambiguation of acronyms, which are widely used in this domain and propose a challenge due to the ambiguity of the meanings which are typically dependent on the context.

\subsection{Negation}

\subsubsection{The problem}

Electronic Health Records contain valuable information from patients, written in narrative form in free-text. In order to extract knowledge from an EHR, the free-text written has to be analyzed. One of the key characteristics of narrative texts in the healthcare sector is that it commonly 
encloses negated concepts. According to [Chapman et al., 2002], around half of all the clinical conditions in these EHRs are negated.

The fact that half of the clinical conditions mentioned typically appear in negative form, implies a whole challenge to detect the sense of what is written. This problem is easily solvable by a human, but can be rather complex for an algorithm. Under certain circumstances, humans also may not agree on the positivity or negativity of a particular term, specially in complex sentences where several items are mentioned preceded by a negative indicator.

Section 3.2.2 presents other existing approaches for detecting negation in general and clinical texts. Section 3.2.3 introduces our solution to the aforementioned problem.

\subsubsection{State of the art}

Several systems and methods have been proposed for negation detection [Chapman et al., 2001], [Mutalik et al., 2001], [Chapman et al., 2002], [Elkin et al., 2005], [Huang and Lowe, 2007], [Rokach et al., 2008], [Morante et al., 2008], [Harkema et al., 2009], [Morante and Daelemans, 2009], [Skeppstedt, 2011], [Skeppstedt et al., 2011], [Chapman et al., 2013].

NegEx is introduced in [Chapman et al., 2001] to identify those negation terms, also called triggers. In order to find negated terms in the text, NegEx uses regular expressions to find and determine the scope of the negation. NegEx was applied to selected sentences from discharge summaries with 0.945 specificity, 0.845 precision and 0.780 sensitivity. In [Chapman et al., 2002], NegEx performance is evaluated with different types of clinical reports and types of sentences, containing different kinds of trigger elements for negation (before and after an Unified Medical Language System (UMLS) concept) and pseudo-negation and other narrative forms. NegEx obtained on average a precision of 0.970 , but ranging from 0.84 to 0.19 depending on the section of the pathology reports analyzed.

More recently, the challenges in the translation of this algorithm and their triggers to other languages has been analyzed in [Chapman et al., 2013]. OWL and RDF multilingual lexicons were developed based on an extended NegEx version proposed in [Harkema et al., 2009].

This extended version, called ConText [Harkema et al., 2009], is also based on regular expressions and adds more narrative forms to the solution, identifying if a particular condition is negated, in hypothetical form, or if it corresponds to an historical item in the patients' history or if the entity found is experienced by any other person different from the patient itself.

There are also mentions to the use of NegEx based on the work performed in other projects like Deteami, a software developed for pharmacosurveillance [Casillas Rubio et al., 2016]. In [Santiso et al., 2017] an study with 75 EHRs is done with an F-measure of $76.2 \%$ for entity recognition, and $73.8 \%$ for the negation detection of those entities. In [Marimon et al., 2017] the IULA Spanish Clinical Record Corpus is presented and an study on the annotation of negation is done. In [Diaz et al., 2017] an approach to annotate negation in Spanish Clinical Texts is also introduced.

Skeppstedt [Skeppstedt, 2010], [Skeppstedt, 2011], [Skeppstedt et al., 2011] adapted the 
English rule-based negation detection system, NegEx to Swedish. The results obtained lower precision and recall than the English version. In [Skeppstedt et al., 2011], NegEx is adapted to analyze SNOMED-CT terms included in the categories 'finding' or 'disorder' from free-texts gathered from the Stockholm EPR corpus. More recently in [Skeppstedt, 2014] the analysis focuses on four different categories: Disorder, Finding, Pharmaceutical Drug and Body Structure.

A more recent translation of NegEx into the German language is presented in [Cotik et al., 2016], with results of 0.96 for accuracy and a precision of 0.88 applying the solution to discharge summaries and 0.95 for clinical notes and a precision of 0.95 in negation detection.

Negation detection is not only done by NegEx, but also by other projects that have arise in the last decades. Negfinder [Mutalik et al., 2001] presents identification of negated patterns that appear in medical documents. These documents have been annotated using UMLS and changed the string representing a concept by their Concept Unique Identifier (CUI). The sensitivity and specificity obtained from evaluation when Negfinder was applied to medical documents to detect negations were between 0.91 and 0.96 .

More complex approaches have been made combining the easiness of regular expressions with parsing to detect negation in radiology reports [Huang and Lowe, 2007]. Reported sensitivity and precision values are of 0.926 and 0.986 respectively.

Machine learning plays an important role also in the detection of negated terms. Several projects have been proposed using this approach [Rokach et al., 2008], [Morante et al., 2008], [Morante and Daelemans, 2009]. The scope of negation in biomedical texts with two classifiers is introduced in [Morante et al., 2008] and an extension using different texts was performed in [Morante and Daelemans, 2009].

Automatic detection of negation in medical narrative texts was done in [Rokach et al., 2008]. The accuracy reported is improved with respect to other methods based on machine learning, thanks to their four steps integrating the method: corpus preparation, regular expression pattern learning, patterns selection and classifier training.

\subsubsection{Solution}

As it has been stated before, the proposed solution to solve negation detection in Spanish written medical texts was based on the translation of the triggers introduced in NegEx algorithm for English written texts. Up to our knowledge, this was the first approach to the problem applied to Spanish biomedical texts.

In the following sub-sections the experiments conducted and the dataset used are introduced.

\section{Preliminaries}

NegEx algorithm [Chapman et al., 2001] uses regular expressions and a list of triggers (words indicating negativity) to determine if clinical conditions are negated. In NegEx there are two 
types of triggers:

- Negation terms which are the ones that indicate negation. Three different categories are found:

- Pseudo negation terms, which are the ones looking like negation terms but are not negating the clinical condition. These are skipped in the algorithm until another negation term is found.

- Pre-condition negation terms, which are the negation terms found before the term they are negating. These are used in Regular Expression 1.

- Post-condition negation terms, which are the negation terms found after the term they are negating. These are used in Regular Expression 2.

- Termination terms which are the indicators of the end of the scope of the negation.

These terms are found in the text by using two different regular expressions:

- Regular expression 1: $<$ negation term $><\$><$ termination term or end of sentence $>$

- Regular expression 2: $<$ clinical condition $><\$><$ negation term $>$

\$ represents a specified or unspecified number of words. The pseudocode of the NegEx algorithm can be found in Listing 3.1.

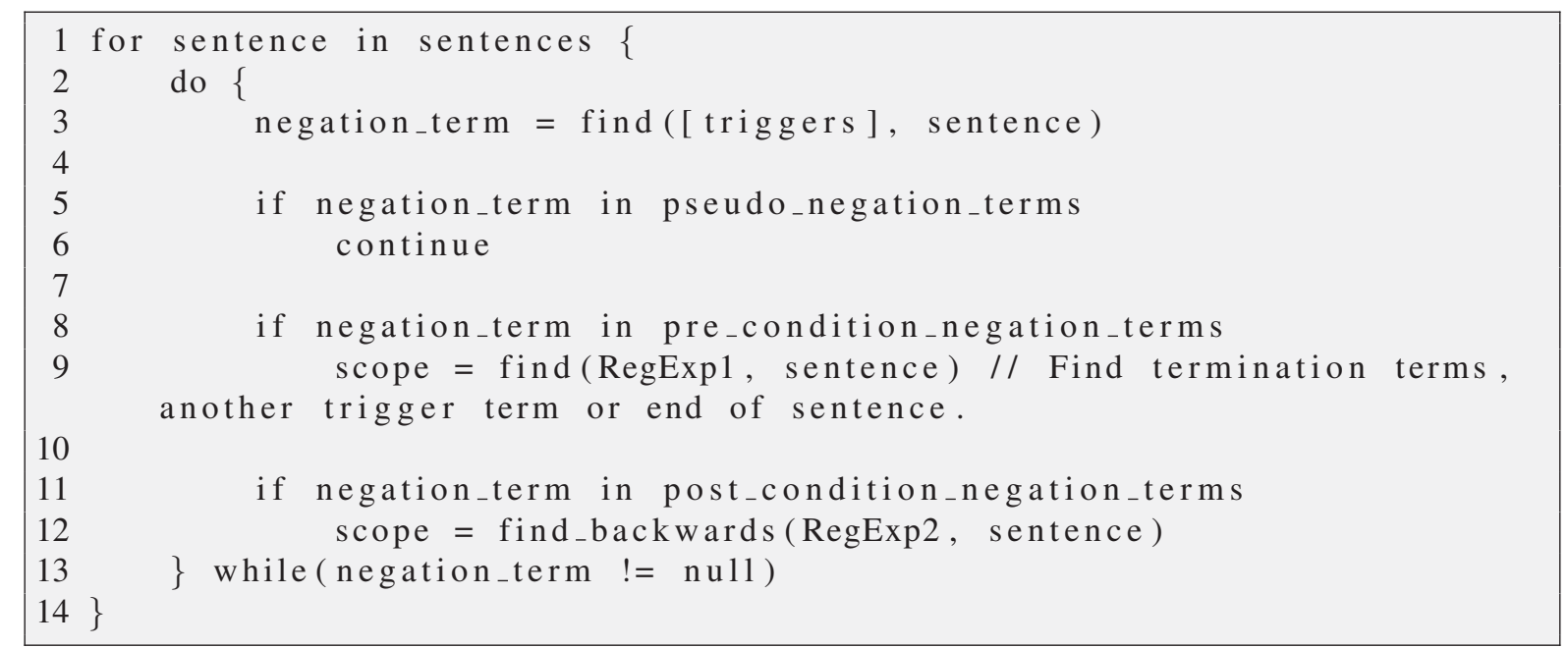

Listing 3.1: Pseudocode of the NegEx algorithm

The algorithm iterates over all the sentence in a document and over all the negation terms in each sentence. In line 3, the next negation term is found from the list of triggers, which is ordered from smallest to the largest terms. If the negation term is a pseudo negation term, the algorithm skips to the next term (lines 5-6). If the negation term is a pre-condition negation term, the scope is determined using the Regular Expression 1, by finding termination terms, 
another trigger term, or a character indicating the end of sentence (lines 8-9). If it is a postcondition negation term, the scope is found backwards (lines 11-12).

The following sentences show examples of the output of the algorithm, with the trigger terms in bold affecting the enclosed terms:

The patient does not suffer from $\{$ headache and has $\}$ no $\{$ dysnesia $\}$.

The patient denies \{chest pain $\}$ but has suffered from shortness of breath.

$\{$ The patient is tumor\} free.

In the research performed, a new asset has been developed by translating the triggers from NegEx algorithm and adding synonyms and other typical words used in Spanish inflecting negation. An example of the terms used can be seen in Table 3.1. In this asset, not only the terms used in [Chapman et al., 2001], but also the ones from ConText [Harkema et al., 2009] are translated.

\begin{tabular}{|c|c|}
\hline Term in English & Term in Spanish \\
\hline $\begin{array}{c}\text { can be ruled out } \\
\text { can rule him out } \\
\text { can rule out }\end{array}$ & se puede descartar \\
\hline no, not & no \\
\hline no evidence & $\begin{array}{c}\text { sin evidencia } \\
\text { no evidencia }\end{array}$ \\
\hline no news & sin novedad \\
\hline no support for & no hay soporte para \\
\hline no suspicion of & ninguna sospecha de \\
\hline not had & no tenía \\
\hline
\end{tabular}

Table 3.1: Example of the translated terms and synonyms added

\section{Dataset}

The dataset collected for the experiments has been gathered from SciELO [Packer, 1999], by extracting 500 reports with the entitled sections: "Reporte de caso" (Case report), "A propósito de un caso" (About a case), "Caso clínico" (Clinical case), and other similar ones. This dataset contains 500 documents with 422 different sentences and 267 unique clinical conditions.

A summary of the dataset:

- Source: SciELO

- Type of documents: Clinical reports

- \# of words: 1,164,712 (65,605 unique) 
- \# of unique clinical conditions: 267

- \# of sentences: 422

- \# of documents: 500

- Availability: Documents freely available at http://www.scielo.org/php/index.php?lang=es

\section{Experiment}

The experiment developed follows the same aspects of the directives explained in [Chapman et al., 2013].

\section{Evaluation}

The dataset introduced was manually annotated to determine the appearance of clinical conditions. These conditions are manually tagged as positive or negative form in their corresponding sentence. After the manual annotation is performed, the dataset was pre-processed by indexing the sentences using Apache Lucene [The Apache Foundation, 2000]. ICD-10 [Organization, 2006] was used as the standard to identify the clinical conditions mentioned in the texts.

During the development of the experiment, NegEx algorithm has not essentially been changed and only the adaptation of the triggers was done, so this experiment focuses on the analysis of the use of the algorithm in Spanish written texts.

The processes in Figure 3.1 have been performed:

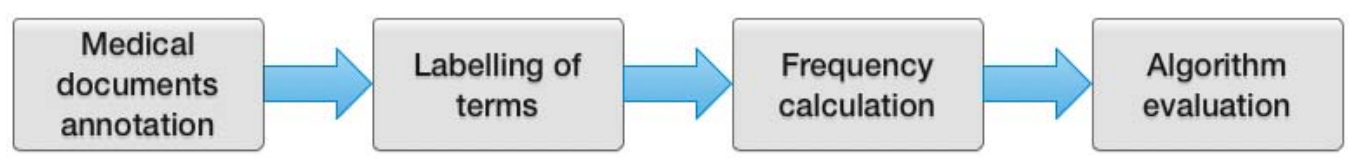

Figure 3.1: Approach followed in the experiment of negation detection

Medical texts annotation: The documents included in the dataset have been manually annotated to detect medical conditions and their status (positive or negative).

Labelling of terms: NegEx trigger terms are collected and translated into Spanish. The ones used in ConText also are collected and translated. The final list is enriched with other terms that have been previously detected in the documents and other known synonyms. The process is depicted in Figure 3.2.

Frequency calculation: The frequency of the terms is calculated to categorize them. This makes it possible to compare the most frequently used terms in Spanish to those in English. According to [Chapman et al., 2013], each translated term is assigned a certain category: I) Definite Negated Term, II) Probable Negated Term, III) Pseudo Negated Term. 
Chapter 3. Addressing specific challenges in the application of Natural Language Processing for Spanish Electronic Health Records

3.2. Negation

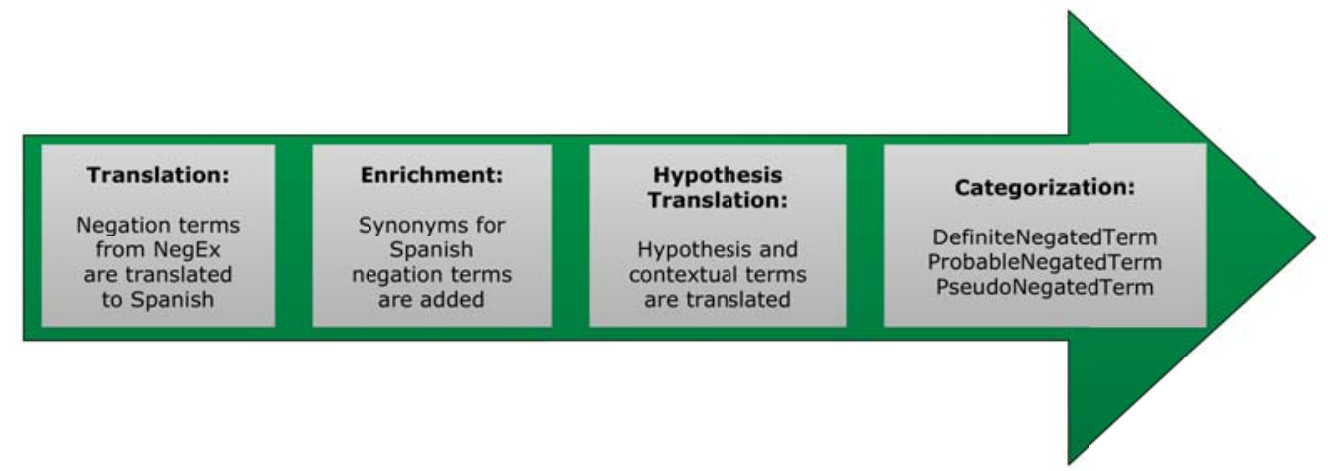

Figure 3.2: Phases of the labelling process

Once all the sentences of the corpus are categorized, the frequency of each term of the lexicon on the corpus is calculated. The median value of the frequency for each term is also calculated and then the terms are categorized as follows:

- No appearance: For those terms with frequency equal to zero.

- Infrequent: Used to categorize those terms which frequency is greater than zero but lesser than the median value.

- Frequent: Those terms that appear with frequencies higher than the median value.

Evaluation: NegEx algorithm is executed with the list of trigger terms collected and the dataset, whose performance is then evaluated.

\section{Lexical Analysis}

Tables 3.2, 3.3 and 3.4 show the relative frequencies for terms both in Spanish and English which demonstrate Definite Negated Existence, Probable Negated Existence and Pseudo Negated Existence, respectively.

\begin{tabular}{|l|l|l|l|l|l|}
\hline \multicolumn{2}{|l|}{ Spanish (58) } & \multicolumn{3}{l|}{ English (60) } \\
\hline Frequent & Infrequent & No appearance & Frequent & Infrequent & No appearance \\
\hline 14 & 20 & 23 & 4 & 24 & 32 \\
\hline
\end{tabular}

Table 3.2: Definite Negated Terms frequencies in Spanish and English

\begin{tabular}{|l|l|l|l|l|l|}
\hline \multicolumn{4}{|l|}{ Spanish (50) } & \multicolumn{3}{l|}{ English (78) } \\
\hline Frequent & Infrequent & No appearance & Frequent & Infrequent & No appearance \\
\hline 4 & 17 & 29 & 3 & 37 & 38 \\
\hline
\end{tabular}

Table 3.3: Probable Negated Terms frequencies in Spanish and English 
Chapter 3. Addressing specific challenges in the application of Natural Language Processing for Spanish Electronic Health Records 3.3. Detection of UMLS entities and drug names

\begin{tabular}{|l|l|l|l|l|l|}
\hline \multicolumn{2}{|l|}{ Spanish (18) } & \multicolumn{3}{l|}{ English (16) } \\
\hline Frequent & Infrequent & No appearance & Frequent & Infrequent & No appearance \\
\hline 1 & 13 & 4 & 2 & 13 & 1 \\
\hline
\end{tabular}

Table 3.4: Pseudo Negated Terms frequencies in Spanish and English

\section{Validation of NegEx implementation for Spanish}

The Spanish version adapted of NegEx using the aforementioned dataset shows the following performance values: precision of 0.494 , recall of 0.557 , and F-measure of 0.523 in the detection of definite negated terms. The process obtained an accuracy of 0.833 .

In the task of detecting elements of positive mentioned terms, a precision of 0.868 , a recall of 0.952 , and a F-measure of 0.908 , were obtained. The accuracy has a similar value as before with 0.847 .

In order to compare these results with other versions of NegEx for different languages, a comparison has to be made. NegEx algorithm according to [Chapman et al., 2001] presents a specificity of 0.945 in a test with 1000 sentences and 1235 medical concepts. Swedish adaptation [Skeppstedt, 2011] shows a precision of 0.752 and a recall of 0.819 with 558 sentences. NegEx in French [Deléger and Grouin, 2012] and has shown a recall 0.85 and a precision of 0.89 using cardiology notes.

In both studies, the adapted NegEx systems achieved comparable recall to the English NegEx with observable differences in precision (differences of $-9.3 \%$ and $4.4 \%$, respectively). Error analyses from these studies suggest that increasing lexicon coverage, improving scope detection, and including uncertainty assertion could boost the algorithm's porting performance [Chapman et al., 2013].

\subsection{Detection of UMLS entities and drug names}

\subsubsection{The problem}

Automatic annotation of EHRs is a challenge as this type of documents contain a very specific vocabulary. Entities such as diseases, parts of the body, or signs and symptoms, can be found among others over different parts of the free-text with relevant information.

UMLS [Humphreys and Lindberg, 1993] is one of the most widely used ontologies that contains a hierarchy of these types of entities. Therefore, this ontology can be used to extract the relevant information contained in medical documents, to annotate the different concepts mentioned in EHRs. UMLS also allows to link information at different layers and with each of the external sources UMLS has used to be built.

Among other entities, drug names are also found. Drug mentions which offer key in- 
formation about what the patient is taking, and the treatment that it should follow after the consultation. One of the biggest challenges regarding drug mentions is the detection of the drug doses to be taken by the patient. This varies a lot depending on the type of drug to be taken as drugs are sold in different formats: pills, effervescent pills, liquid.... Drug doses can be simple: $375 \mathrm{mg}, 100 \mathrm{ml}$; compound: $375 \mathrm{mg} / 100 \mathrm{ml}, 300 \mathrm{mg} / 125 \mathrm{mg}$; time dependent: $1 \mathrm{pill} / 8 \mathrm{~h}$, 1-0-1 (every $12 \mathrm{~h}$ breakfast and dinner).

Another of the challenges found in drug mentions detection is the huge difference among different prescriptions of the same drug, and the way they are commercialized by different laboratories. A drug is formed of active principles and excipients and doctors can either write an excipient, the active principle, or the concrete prescription as it is sold in the pharmacies and drug stores. This problem adds a lot of complexity to the detection of drug mentions as the variability and possibilities are endless.

Moreover, drug entity recognition plays an important role, as its recognition can be used to determine drug interactions, which is one of the areas of more interest to the healthcare community.

Many of the tools developed in the field of NLP for EHRs, from the most complex to the simplest ones, contain some part of NER applied to the analysis of the entities mentioned, which are the main source of information.

In this section the challenge of automatically annotating medical entities and drug names using the UMLS ontology and other resources is addressed. First, an analysis on the performance of two of the most well-known tools to perform NER in English, MetaMap and Clinical Text Analysis and Knowledge Extraction System (cTAKES), is presented. These tools are used to annotate entities in English for 30 infectious diseases extracted from MedLine Plus. Finally, a general method to improve the annotation of medical entities, mitigating the ambiguity and the definition of their scope to be used in Spanish EHRs is introduced.

\subsubsection{State of the art}

There exists a good amount of medical knowledge which is published digitally in websites, databases and ontologies. These resources are often used when trying to find entities in medical texts. In the following subsections, the state of the art regarding NER is reviewed: generic NER in the healthcare domain, drug name extraction related projects, and existing NLP frameworks to perform NER.

\section{Named Entity Recognition in healthcare}

Some of the available resources on the web may lack of reliability as many of them are community supported and the contributions are made by users whose reputation on the topic cannot be assessed. The most relevant example of community supported website is Wikipedia [Foundation, 2001]. There are many articles regarding medicine in Wikipedia which may be used to extract contextual information to recognize entities. Other example of community supported 
network is Freebase [Bollacker et al., 2008], a collaboratively created graph database to structure all kinds of human knowledge, including medical knowledge.

Another example of collaborative website is MedicineNet [Inc., 1996], which was created in 1996. This website indexes articles regarding healthcare and IT related articles to provide healthcare-related information. The information is proposed by doctors and physicians, rather than being an open-collaborative website.

The main problem faced by these websites is that they typically do not offer structured end-points to gather the information they contain, so free-text has to be analyzed, ending up in the same problem trying to be solved. Other of the most renowned websites is MedLine Plus [DAB, 1999], maintained by the U.S. National Library of Medicine with information about diseases, procedures, symptoms, etc. This website also offers the information in Spanish.

These websites propose generic information about medical topics but there are specific resources on different domains, such as Online Mendelian Inheritance in Man (OMIM) [Hamosh et al., 2002], a compendium of human genes and genetic phenotypes, as well as the Human Phenotype Ontology (HPO) [Köhler et al., 2014]. The AlzGene database [Bertram et al., 2007] gathers information about the genetic association with Alzheimer's disease. The PDGene database [Lill et al., 2012] offers a comprehensive view on the genes affecting in the development of Parkinson's disease. Focused on genetic information and the genes variants is also DIDA: a curated and annotated digenic diseases database [Gazzo et al., 2016].

Ontologies have also been created to propose hierarchical information and more structured and trusted information. One of the most used ontologies is Systematized Nomenclature of Medicine - Clinical Terms (SNOMED-CT) [International, 2017], an ontology created by the organization SNOMED International with the aim to contain information about clinical terms. Logical Observation Identifier Names and Codes (LOINC) [Forrey et al., 1996] is another proposed structured database for laboratory observations and related entities.

Medical Subject Headings (MeSH) [Lowe and Barnett, 1994] proposes hierarchicallyorganized terminology cataloging biomedical information gathered from the U.S. National Library of Medicine. There are also international efforts to codify diseases and procedures such as the International Classification of Diseases (ICD) [Organization et al., 2012], which is now on its tenth revision but the ninth is still widely used.

Following these efforts, the U.S. National Library of Medicine also takes care of UMLS [Humphreys and Lindberg, 1993]. This proposed meta-ontology gathers information from different sources (MeSH, LOINC, SNOMED-CT, etc.) to integrate and distribute key terminology, assigning proper CUIs to follow a coding standard. Therefore, UMLS gathers relevant information in the biomedical field which is commonly used in different NLP projects in the field. A further explanation on how UMLS annotates entities is described in Section 3.3.3.

Some other works have explained the relevance of using NLP to extract entities in the healthcare domain. One of this examples is [Demner-Fushman et al., 2009], where an analysis on the relevance of standardizing medical nomenclature is done. Different studies show the relevance of using NLP in order to perform proper NER detection. In [Ruch et al., 2003], a study is performed to recognize entities and improve the spelling correction of the typos written 
Chapter 3. Addressing specific challenges in the application of Natural Language Processing for Spanish

Electronic Health Records

3.3. Detection of UMLS entities and drug names

in EHRs. More recently, a study on the challenges of performing NER in EHRs has been done, stating many of the problems mentioned in this thesis [Kundeti et al., 2016].

Some specific studies have also been done by using NLP in order to extract symptoms or other relevant information. In [Jackson et al., 2017] a project to extract symptoms of mental illnesses from free text is introduced. In [Casillas et al., 2016] a system able to extract adverse drug reaction events from EHRs written in Spanish is presented.

\section{Named Entity Recognition for drug names}

Regarding the extraction of entities related to drug names, on 2000, a project named EDGAR [Rindflesch et al., 2000], presented a vision of extracting drugs, genes and relations from the biomedical literature. This NLP software used an stochastic PoS tagger, generating an syntactic parse by using MedLine and UMLS to provide semantic information on these topics. Another approach introduced in [Narayanaswamy et al., 2003] presents a more general NER extraction system for biological texts, specially focused on the analysis of chemical names and their automatic extraction. A more recent review on approaches and applications of chemical NER is presented in [Eltyeb and Salim, 2014].

In [Segura-Bedmar et al., 2008] a novel approach for detecting drug names is presented. In this project, biomedical texts are examined by using UMLS, MetaMap Transfer, and the World Health Organization (WHO) International Non-proprietary Names program to identify and classify pharmaceutical substances. Hettne et al. [Hettne et al., 2009] introduced an specific dictionary to identify small molecules and drugs mentioned in free text. This dictionary was created by combining information from UMLS, MeSH, ChEBI [Degtyarenko et al., 2008], DrugBank [Wishart et al., 2008], KEGG [Kanehisa and Goto, 2000], HMDB [Wishart et al., 2007] and ChemIDplus [Tomasulo, 2002].

Recent approaches on the use of NLP in order to provide drugs NER have been developed. Machine learning approaches to recognize chemical names within large text databases have also been studied in [Wren, 2006]. In this study, a first order Markov model was evaluated to distinguish chemical names from words, yielding 93\% recall in recognizing chemical terms and $99 \%$ precision in rejecting non-chemical terms on smaller test sets. The scalability of the proposed solution was tested with more than 13 million records from MedLine.

In [Boudin et al., 2008] statistical and symbolic approaches are studied in the field of chemical names recognition. Chemspot [Rocktäschel et al., 2012] introduces a Condition Random Field combined with a dictionary to provide NER mainly focused on the extraction of chemical entities. This system achieved a $68.1 \%$ F-measure on SCAI corpus. The authors compare their results which outperform other known software called OSCAR4 [Jessop et al., 2011] which implements an architecture for detecting chemicals. Leaman et al. [Leaman et al., 2015] report a state of the art tool called tmChem for chemical NER which, according to the authors, equals or outperforms the tools previously existing. In [Korkontzelos et al., 2015], a voting system based on dictionary knowledge and gold-standard corpora was used with 120 abstracts. Genetic programming to evolve regular expressions to capture common drug suffixes was used. 
But most of the studies in the recent years, not only focus on the NER of drugs as a general use case, but rather focus on the drug extraction in order to analyze drug-drug interactions. The study performed by Jiao et al. [Jiao and Wild, 2009] focus on the use of NLP to extract CYP proteins and chemical interaction from journal articles. In [Björne et al., 2013] a machine learning approach is introduced to detect drug mentions as well as their interactions with reported F-measures of $60 \%$ for drug entity recognition and $59 \%$ for the drug interactions. In [Segura Bedmar et al., 2013] a contest is introduced with two tasks in mind: I) The recognition and classification of drug names; II) The extraction and classification of drug interactions.

Another interesting use case is the extraction of potential adverse drug events reported in EHRs as proposed in [Gurulingappa et al., 2012], where the analysis of machine learning approaches for the extraction of potential adverse drug reactions is analyzed against MedLine case reports. In [Aramaki et al., 2010] a first approach is done to detect adverse drug effects reporting that $7.7 \%$ of records include adverse event information, and that automatic tools help to identify $59 \%$ of them.

These tools presented rely on different dictionaries or ontologies. Some of the studies, have presented new dictionaries of drugs based on existing ontologies like UMLS and MeSH, while others directly on them to extract chemical and drug names. A very specific database on drugs was introduced in [Liu et al., 2005]. RxNorm was created to store structured and normalized drugs information. The U.S. National Library of Medicine create this nomenclature in order to standardize drug names and to assure patient safety.

Up to our knowledge there is no published research on the recognition of drug entities in Spanish. However, some resources are available and AEMPS, the Spanish agency for drugs and healthcare products, supports a complete database with the drugs sold in Spain [de Medicamentos y Productos Sanitarios, 2013]. This database, contains information about the different prescriptions, laboratories, active principles, excipients, and drugs interactions and adverse effects.

\section{NLP frameworks used to perform Named Entity Recognition}

More generic projects have been developed in order to detect entities by using NLP methods. These projects typically offer complex architectures that are able to extract knowledge from the clinical texts. Two of the most broadly studied tools are MetaMap [Aronson, 2001] and cTAKES [Savova et al., 2010], which are freely available to the public and make it possible to discover medical entities in text and map them to ontology concepts.

As many of the resources that have been mentioned, MetaMap has been developed by the U.S. National Library of Medicine. MetaMap identifies entities and maps them to their corresponding UMLS concepts. This tool is very tightly coupled with UMLS but yet is very flexible and customizable. However, decoupling MetaMap from UMLS is possible, but several structures have to be always present. Unfortunately, this adaptation to use different dictionaries is not always possible [Bhatia et al., 2009].

cTAKES is an open-source supported by the Apache Foundation. This project is focused 
on the extraction of information from clinical documents. Built on top of the UIMA framework [Ferrucci and Lally, 2004] and using OpenNLP [OpenNLP, 2011], detects entities with the help of UMLS. The main cTAKES components are sentence boundary detectors, tokenizers, normalizers, Part-of-Speech (PoS) taggers, shallow parsers and Named Entity Recognition (NER) annotators.

The related work shows that current efforts are aligned with the problems identified in this thesis, being NER one of these problems.

\subsubsection{Using MetaMap and cTAKES for English Named Entity Recogni- tion}

In this section, a methodology to extract diagnostic knowledge and recognize medical entities in English written documents by using MetaMap and cTAKES is presented. The following subsection explains the UMLS coding system in order to understand the entity recognition process.

\section{UMLS coding system}

UMLS [Humphreys and Lindberg, 1993] is an metathesaurus created from many different sources that tries to solve the problems in concept ambiguity by creating an structured system for coding medical entities. In UMLS, each medical entity is defined in four different levels of specification:

Concept Unique Identifier (CUI) Defines an identifier for a concept, which is a meaning. Each meaning can have different names. In this sense, the identifier encloses the same concepts (and synonyms) from different sources that mean the same thing. A CUI contains the letter $\mathrm{C}$ followed by 7 numbers.

Lexical (term) Unique Identifier (LUI) Defines an identifier to link strings that are lexical variants. A LUI contains the letter L followed by 7 numbers.

String Unique Identifier (SUI) Defines an identifier for each string given to a concept name in each language. Each string is case sensitive, so any difference on writing the same concept creates a new SUI. A SUI contains the letter S followed by 7 numbers.

Atom Unique Identifier (AUI) Defines an identifier for each of the concept names or strings for each of the source vocabularies used in UMLS. Every occurrence of a string in each source vocabulary is assigned a unique AUI. If exactly the same string appears multiple times in the same vocabulary, for example as an alternate name for different concepts, a unique AUI is assigned for each occurrence. An AUI contains the letter A followed by 7 numbers.

Figure 3.3 contains an example of the coding system containing all the levels of specification. 
Chapter 3. Addressing specific challenges in the application of Natural Language Processing for Spanish Electronic Health Records 3.3. Detection of UMLS entities and drug names

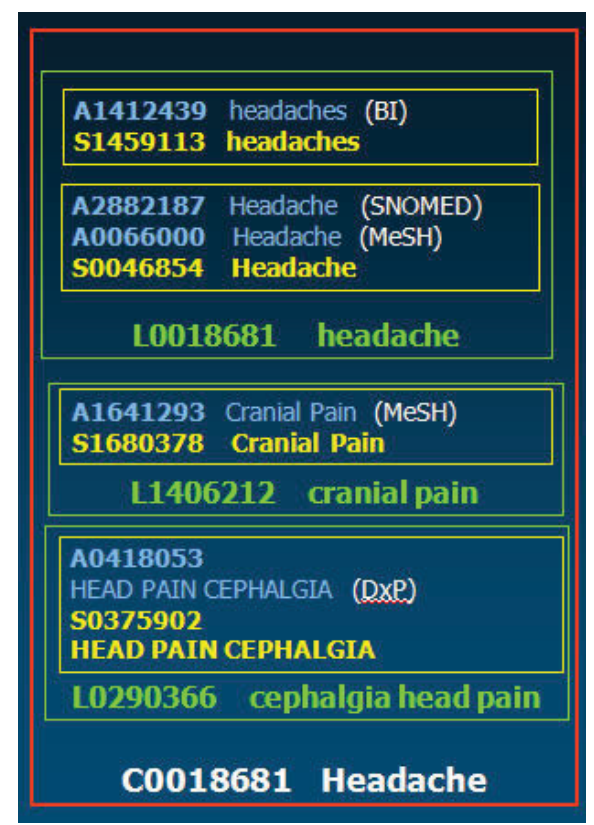

Figure 3.3: Example of the UMLS coding system. Source: UMLS Online Learning

\section{Entity recognition proposed methodology}

The proposed methodology uses MetaMap and cTAKES to find and annotate the entities related to medical terminology. These frameworks gather information from UMLS and other collaborative sources.

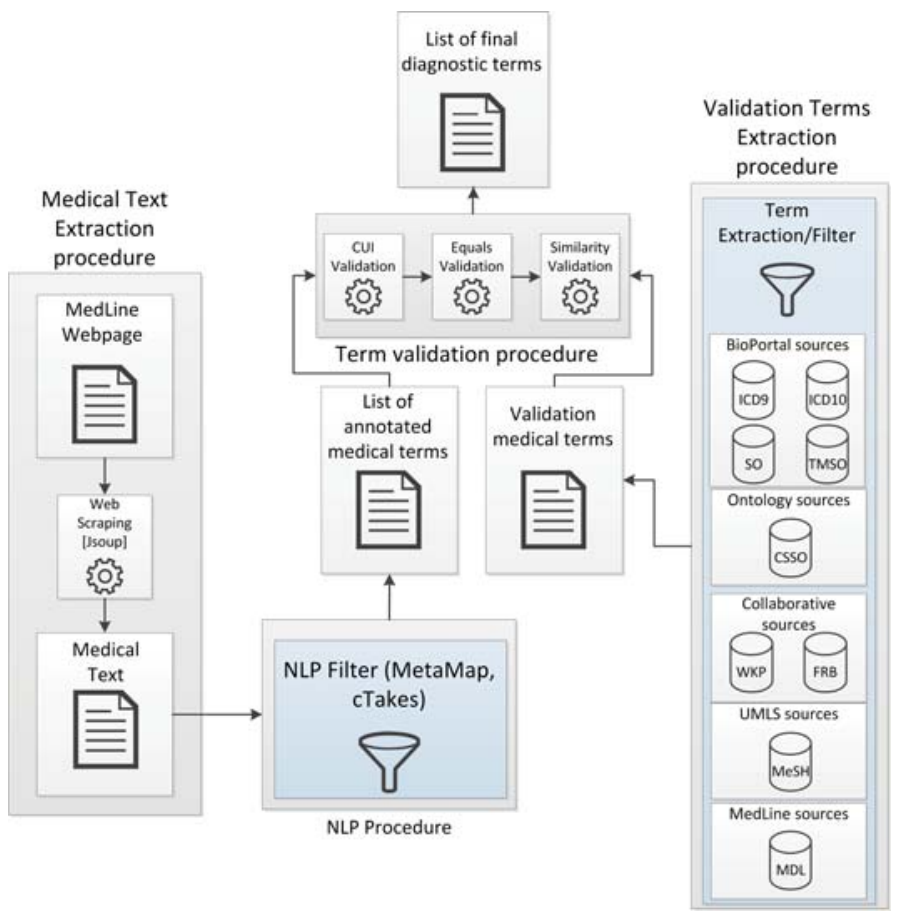

Figure 3.4: Architecture of the proposed solution 
The approach proposed is depicted in Figure 3.4 and defined by:

1. Medical Text Extraction and NLP Procedures (MTENP): Documents are extracted from MedLine Plus for a given disease by scraping the website using JSoup API from the sections marked as relevant for this experiment: "Symptoms" and "Exams and Tests". These documents are then passed to the aforementioned NLP frameworks to identify all the relevant diagnostic-related terms.

2. Validation Terms Extraction Procedure (VTE): Diagnostic knowledge is extracted in this step. Each identified concept extracted from the NLP procedures (MetaMap and cTAKES) has a semantic type assigned. Both frameworks treat the semantic types differently. On the one hand, MetaMap uses "Diagnostic Procedure", "Disease or Syndrome", "Finding", "Laboratory Procedure", "Laboratory or Test Result" and "Sign or Symptom". On the other hand, cTAKES uses only three semantic types for the same categories, which are "Disease Disorder Mention", "Sign Symptom Mention", and "Procedure Mention". The correspondence between the different types on each system can be found in Table 3.5.

MTENP returns a list of medical terms found to be relevant and which are categorized in the aforementioned semantic types.

\begin{tabular}{|c|c|}
\hline MetaMap & cTAKES \\
\hline Diagnostic Procedure (diap) & Procedure Mention \\
\hline Disease or Syndrome (dsyn) & Disease Disorder Mention \\
\hline Finding (fndg) & Sign Symptom Mention \\
\hline Laboratory Procedure (lbpr) & Procedure Mention \\
\hline Laboratory or Test Result (lbtr) & Procedure Mention \\
\hline Sign or Symptom (sosy) & Sign Symptom Mention \\
\hline
\end{tabular}

Table 3.5: Correspondence between MetaMap and cTAKES semantic types

VTE aims to maximize the accuracy of the global approach by comparing terms extracted in the previous phase, with different trusted and not-so-trusted sources different than MedLine Plus. In this case, findings like "red" or "pale" will be filtered out as by themselves they do not offer relevant information.

VTE uses the following publicly available information sources (see Table 3.6), which are classified in four categories:

Trusted sources Created and curated by widely known institutions or organizations, such as WHO. For instance, the International Classification of Diseases 10 - Clinical Modification (ICD10CM) is a classification provided by the Centers for Medicare and Medicaid Services (CMS) and the National Center for Health Statistics (NCHS), for medical coding and reporting in the United States.

Research sources Sources created as part of a research project. Includes: CCSO Signs and Symptoms Ontology, TM Signs and Symptoms Ontology (TM SSO) and Symptoms Ontology. 
Chapter 3. Addressing specific challenges in the application of Natural Language Processing for Spanish Electronic Health Records 3.3. Detection of UMLS entities and drug names

Collaborative sources Mainly Wikipedia and Freebase. Their reliability is, in general, not as good as the sources mentioned above, but the vast amount of information can be used in combination with other sources for validation purposes.

Other sources Other medical webs. Includes: MedicineNet (Tests section).

\begin{tabular}{|c|c|}
\hline Extraction method and origin & Sources \\
\hline Manual (webpage) & MedicineNet \\
\hline Wikipedia & \\
\hline Automatic & \\
\hline (Bioportal OpenLifeData) & ICD9CM, ICD10CM \\
\hline Symptoms Ontology & \\
\hline TM SSO & \\
\hline Automatic (Jena and MQL) & Freebase (MQL) \\
\hline CSSO (Jena) & \\
\hline
\end{tabular}

Table 3.6: Summary of validation sources and extraction method used

3. Term Validation Procedure (TVP): With the terms extracted from step 1 and given the knowledge extracted in step 2, validation is performed. This step matches terms extracted in the MTENP phase with those extracted in the VTE procedure. If TVP finds that match to be positive, the term will be returned as a valid diagnostic term.

Validation is performed as follows:

A term ( $\mathrm{t}$ ) from MTENP is compared to those in VTE to find a matching term (mt), which is positive if any of the following:

(a) CUI Identification: If the CUI of "t" and "mt" have the same code (e.g. C0015967). This is the most reliable possible match.

(b) String equals: If the string representing " $\mathrm{t}$ " is the same as " $\mathrm{mt}$ ".

(c) Similarity: If the calculated Levenshtein distance [Levenshtein, 1966], using SimMetrics Java API, between " $t$ " and "mt" falls in the threshold value of 0.85 . Both terms are preprocessed to remove stop words, symbols and trimming among other tasks.

VTE procedure follows the priority assigned to the type of sources used to maximize matches from trusted sources. Source code is publicly available at GitHub.

The process carried out by VTE tries to give preference to those validation terms who came, first, from trusted sources; second, from research sources; third, from collaborative sources; and finally from other sources, in order of priority. The source code of the prototype is publicly available at GitHub ${ }^{1}$.

\footnotetext{
${ }^{1}$ https://github.com/alejandrorg/medlineplus2ddx - Last checked: 10-02-2017
} 


\section{Dataset}

The dataset has been gathered to analyze the extraction of relevant information from Medline Plus [DAB, 1999] of symptoms and diagnostic tests regarding infectious diseases.

30 different infectious diseases have been selected manually. These diseases are the ones presented in Table 3.7.

$\begin{array}{ccc}\text { Tonsillitis } & \text { Ebola } & \text { Poliomyelitis } \\ \text { Yellow Fever } & \text { Leptospirosis } & \text { Rabies } \\ \text { Brucellosis } & \text { Cholera } & \text { Rocky Mountain spotted fever } \\ \text { Diphteria } & \text { Erypselas } & \text { Roseola } \\ \text { Q Fever } & \text { Typhoid Fever } & \text { Rubella } \\ \text { Legionnaire } & \text { Tetanus } & \text { Measles } \\ \text { Chickenpox } & \text { Fifth disease } & \text { Scarlet Fever } \\ \text { Hepatitis } & \text { Oral Herpes } & \text { SARS } \\ \text { Impetigo } & \text { Flu } & \text { West Nile Virus } \\ \text { Meningitis } & \text { Mumps } & \text { Pertussis }\end{array}$

Table 3.7: Infectous diseases conforming the dataset

- Source: MedLine Plus

- Type of documents: Diseases web pages

- \# of documents: 30

- Availability: Documents freely available at https://medlineplus.gov/

\section{Results from the experimentation}

The type of diseases selected in the dataset, typically have a large number of symptoms and diagnostic tests.

The evaluation was done by manually analyzing the results provided by the software developed. For each disease given, a comparison was done between the list of the terms automatically provided and the list of terms manually extracted from the diseases web page.

After the comparison was done, confusion matrices were calculated in order to obtain True Positive (TP), False Positive (FP), True Negative (TN), and False Negative (FN) parameters to calculate precision, recall, specificity, and F1 score values.

Figure 3.5 shows the mean results obtained in the executions of the method using MetaMap or cTAKES. The former obtains a higher precision while the latter performs better at recall, being both metrics equally important in such scenarios. The same happens for specificity, where MetaMap performs better, and F1 score, where cTAKES obtains higher values. 
Chapter 3. Addressing specific challenges in the application of Natural Language Processing for Spanish Electronic Health Records 3.3. Detection of UMLS entities and drug names

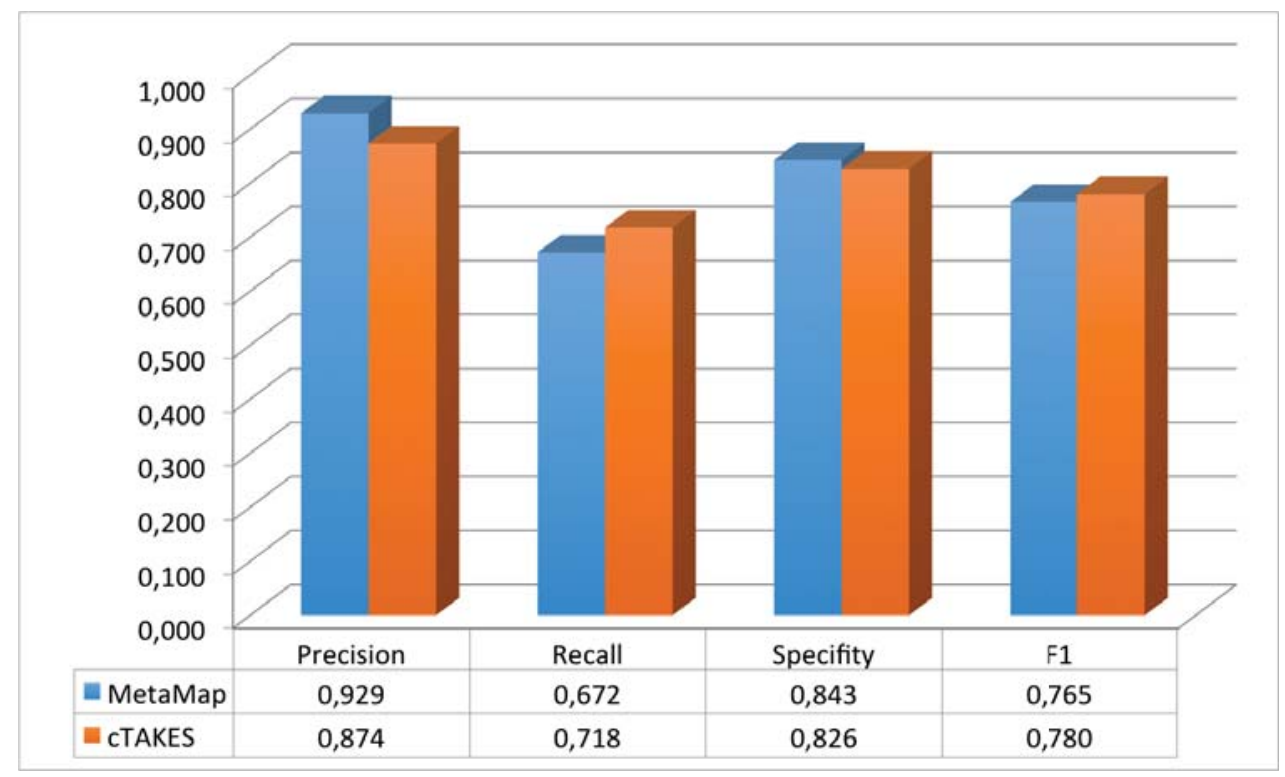

Figure 3.5: Comparison between statistical results for MetaMap \& cTAKES executions

Detailed results for each disease are available online ${ }^{23}$, including the list of terms manually extracted from the disease web page, the matchings with the list of terms provided by our approach and some extra information such as the primary type of source used for the matching.

Some of the false negatives encountered are due to the validation method used in the experiment (specially finding acronyms in diagnostic tests), which made to discard terms that were correctly classified by MetaMap or cTAKES. The method still has some flaws in the NLP process according to the analysis of the terms that have been incorrectly rejected.

When analyzing individual diseases greater differences are encountered. cTAKES typically performs better on laboratory or test results or locating rare symptoms, but increases in most cases the number of false positives, incorrectly annotating several elements as findings. cTAKES also increases true negatives as its NLP process annotates more elements, but these elements are then cleared in the validation which classifies them correctly in most cases.

The results for the different diseases including precision, recall, specificity and F1 scores can be found in Figures 3.6, 3.7, 3.8 and 3.9, respectively.

Figure 3.6 shows that MetaMap outperforms cTAKES in precision in most diseases but 5: Tonsilitis, Erypselas, Fifth disease, Roseola and Scarlet Fever. In most cases this is due to the increase in false negatives and the increase in true positives. For the rest of the diseases, the differences in precision are high enough to ensure the great difference in precision between both systems.

\footnotetext{
${ }^{2}$ https://github.com/alejandrorg/medlineplus2ddx/blob/master/MetaMap.xlsx - Last checked: 10-02-2017

${ }^{3}$ https://github.com/alejandrorg/medlineplus2ddx/blob/master/cTAKES.xlsx - Last checked: 10-02-2017
} 
Chapter 3. Addressing specific challenges in the application of Natural Language Processing for Spanish Electronic Health Records

3.3. Detection of UMLS entities and drug names

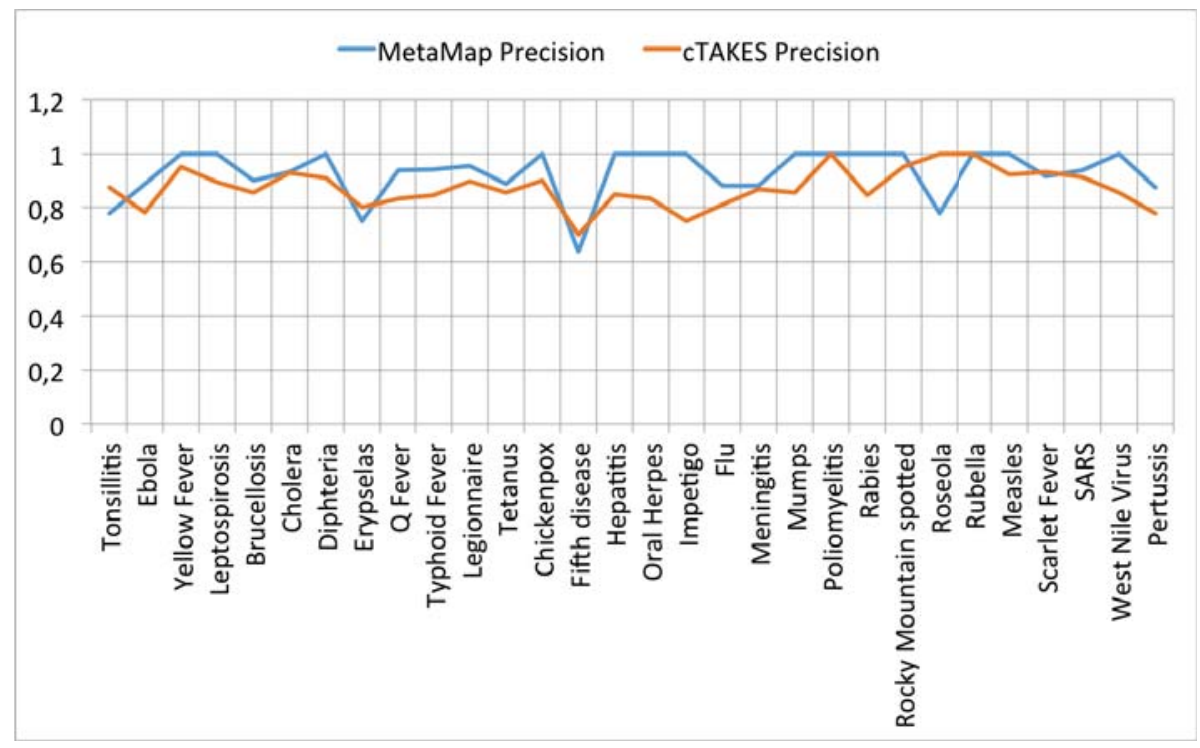

Figure 3.6: Comparison of precision results for MetaMap \& cTAKES executions for each disease.

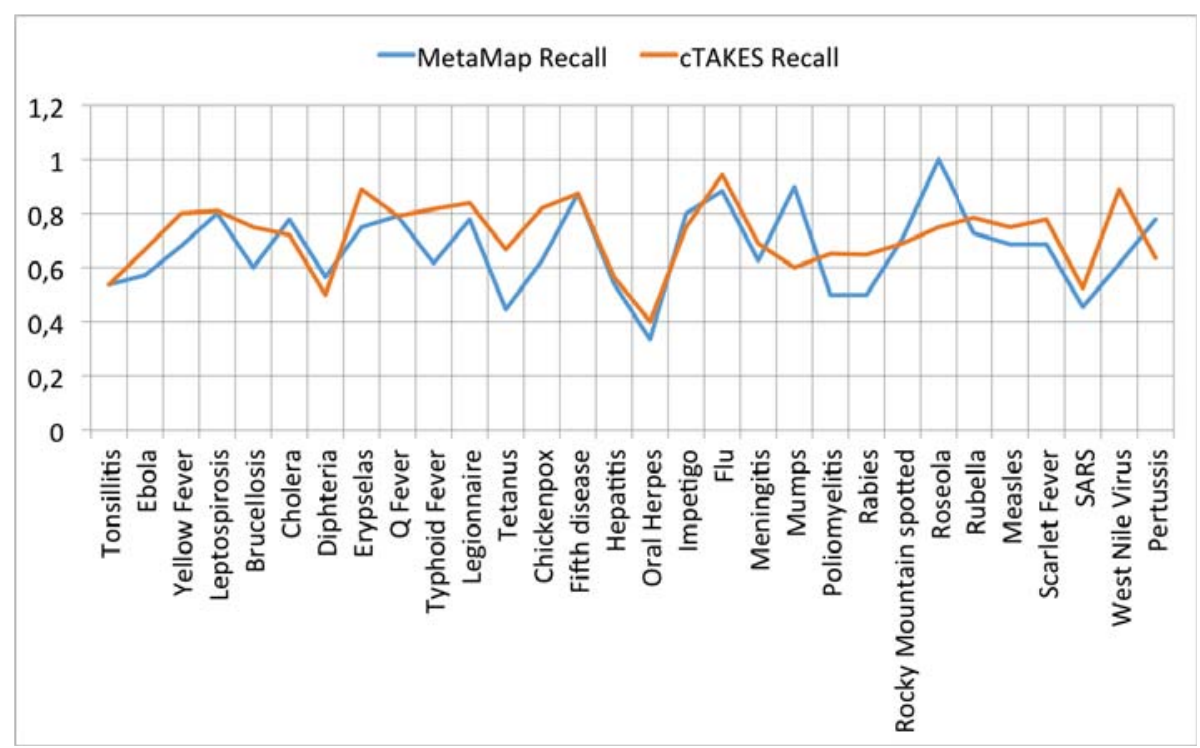

Figure 3.7: Comparison of recall results for MetaMap \& cTAKES executions for each disease. 
Chapter 3. Addressing specific challenges in the application of Natural Language Processing for Spanish Electronic Health Records 3.3. Detection of UMLS entities and drug names

In Figure 3.7 the recall is analyzed using both NLP systems and we observe the inverse effect than the one observed in Figure 3.6, as there are 6 diseases where MetaMap performs better than cTAKES: Cholera, Diphtheria, Impetigo, Mumps, Roseola and Pertussis. In this case the differences are less significant but the improvement using cTAKES is observable.

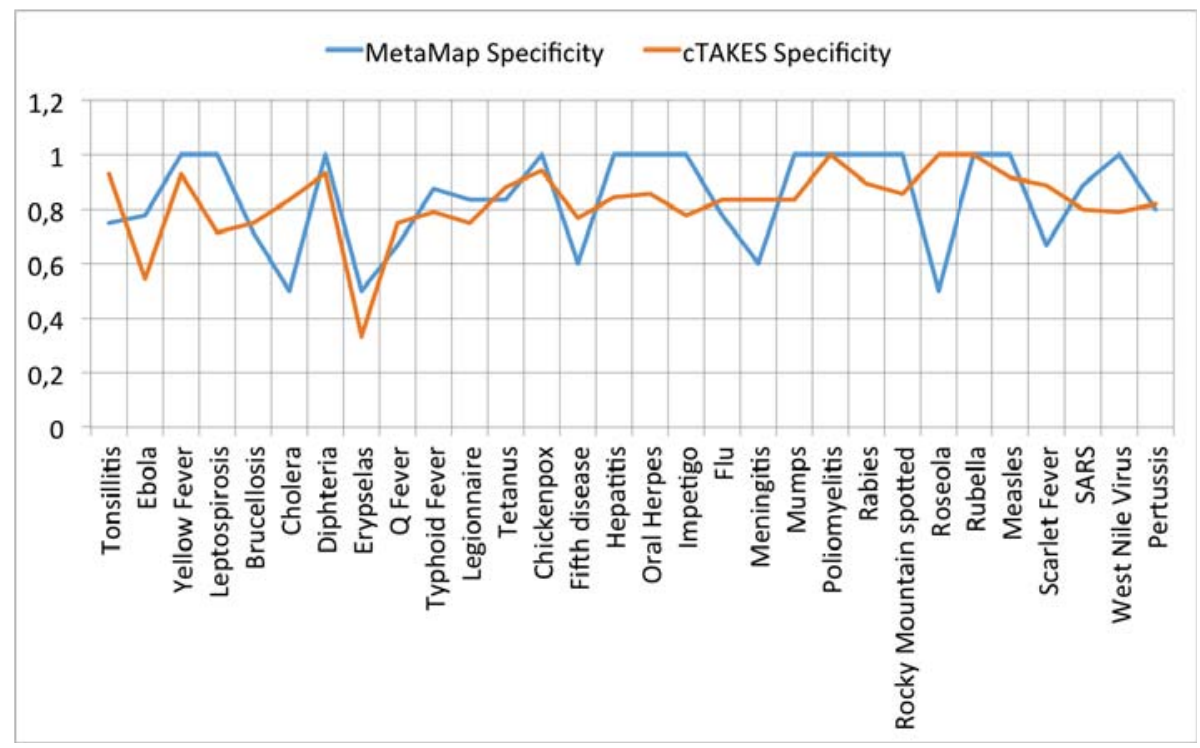

Figure 3.8: Comparison of specificity results for MetaMap \& cTAKES executions for each disease.

In Figure 3.8 the specificity between MetaMap and cTAKES for all the 30 diseases used in our experiments is compared. In this particular case is worth mentioning cases such Poliomyelitis or Rubella, where both systems perform at their best, or the great differences encountered for example in the analysis of Roseola, where the difference in specificity is greater than 0.4 between both systems.

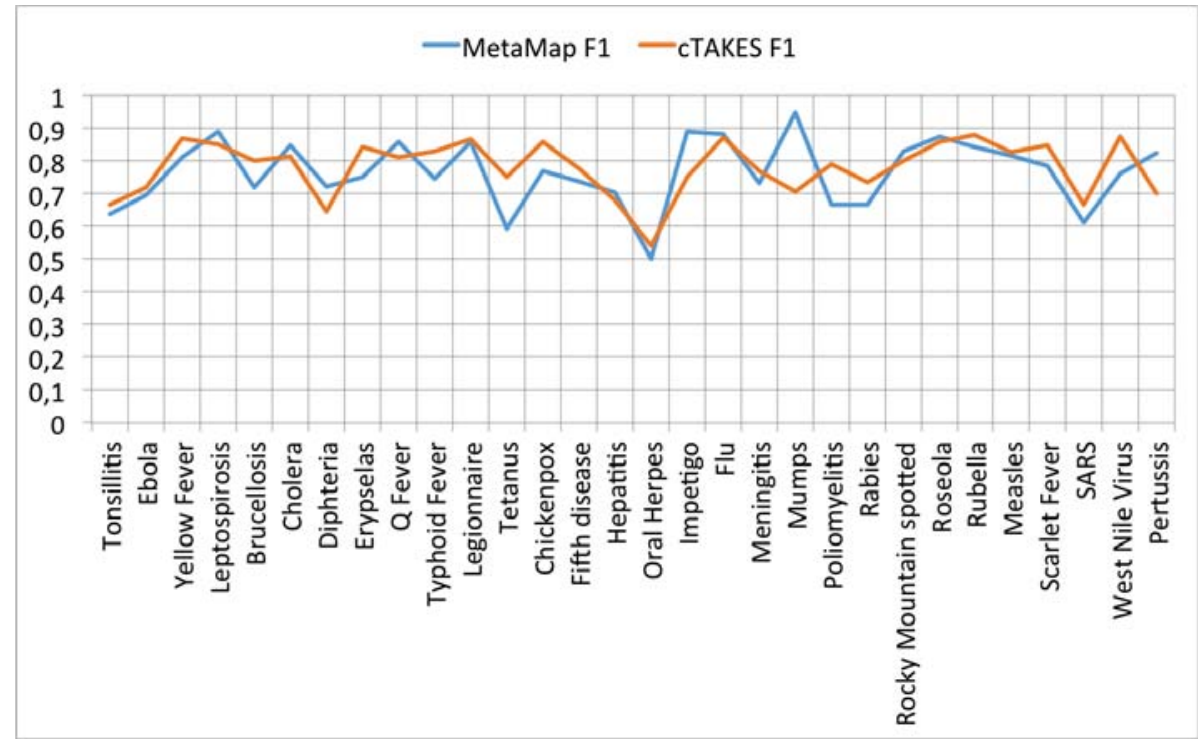

Figure 3.9: Comparison of f1 score results for MetaMap \& cTAKES executions for each disease. 
In Figure 3.9 the comparison between the F1-scores is shown. The lines in this chart are approximately overlapping, meaning that both systems are performing similarly. It is worth noting the two big differences encountered in Tetanus and Mumps with more than 0.2 difference between both systems, performing cTAKES better on the former and MetaMap on the latter.

\subsubsection{Solution: Named Entity Recognition in Electronic Health Records}

Named Entity Recognition is also a problem for structuring Spanish EHRs. In the case of Spanish, one of the main problems to perform NER is the scarce availability of complete dictionaries to serve as a trusted source of information. However, some trusted and more complete sources are available, such as UMLS and ICD in its versions 9 and 10.

Even though the information gathered in UMLS is not complete (compared to that in English), and it does not allow to recognize all of the possible entities, such the drug names mentioned in a EHR, it has has been selected as the main source of information in the proposed methodology. In order to address the NER problem in Spanish EHRs, the information included in UMLS has been complemented with the information extracted from AEMPS [de Medicamentos y Productos Sanitarios, 2013].

The methodology proposed for annotating medical entities from Spanish EHRs is depicted in Figure 3.10.

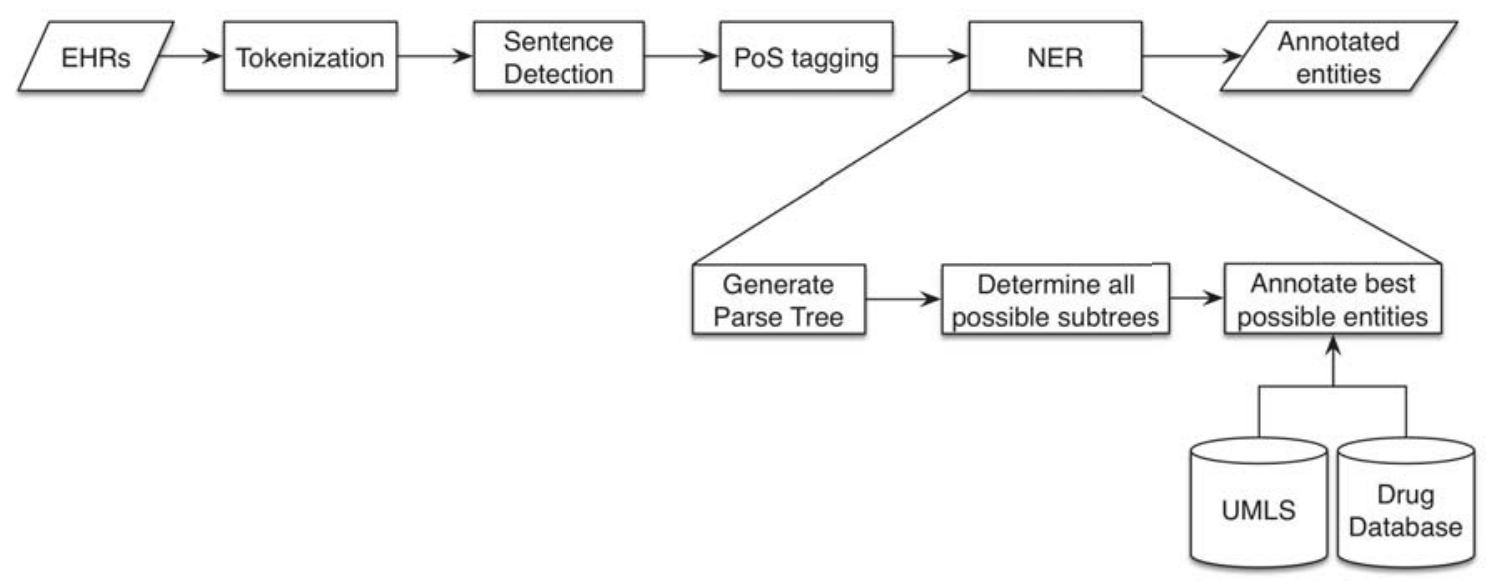

Figure 3.10: Named Entity Recognition process designed

The proposed methodology takes the EHRs as an input which is passed through the typical NLP pipeline: Tokenization, Sentence Detection, and PoS tagging. With the PoS information extracted, NER is performed in three different steps: First, a parse tree is generated with nouns, adjective, numbers and determiners; Then, all possible subtrees containing at least a noun or an adjective are calculated; Finally, the best possible entities gathered from UMLS and the drugs database are annotated. This process outputs the EHRs with the corresponding entities annotated.

In the following subsection, the process of creating the parse tree and the annotation 
process is explained. Then, the solution adopted for maximizing the precision when selecting the possible entities from the sources is detailed.

\section{Annotating entities}

The detection and annotation of entities in a text is complex, specially in the medical domain, as a piece of text can be annotated in different forms, and many partial matches can also be valid, but may not add new knowledge. The generated parse tree to determine the possible subtrees (entities) in a sentence is constructed by putting the first noun found in a sentence as its root, putting to the left the words appearing before it, and to the right the ones after.

This process maximizes the number of entities (prioritizing those to the left of the sentence) to be annotated when a positive match on the results returned from the external ontologies and databases is found. Even though, the tree has been developed to encounter a balance between the size and the number of annotations.

An example will enlighten better this process. Given the sentence "se observa dilatada la arteria cerebral media derecha" and taking into account only names, numbers, determiners and adjectives to create the tree, the result is the one depicted in Figure 3.11.

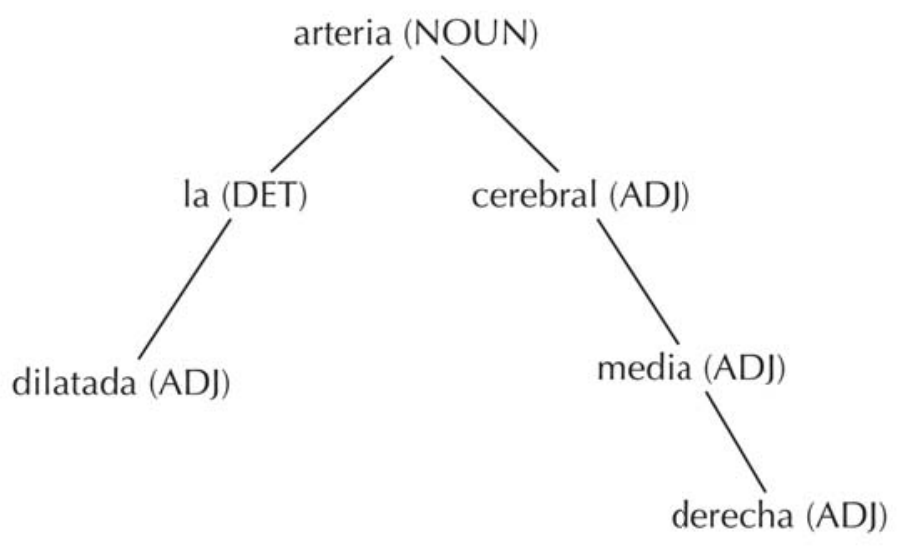

Figure 3.11: Parse Tree created from the sentence "se observa dilatada la arteria cerebral media derecha"

The created tree allows to gather all the possible subtrees (possible entities) that are inside each sentence. The possible generated subtrees, in order of evaluation for the annotation process are:

1. \{dilatada la arteria cerebral media derecha\}

2. $\{$ dilatada la arteria cerebral media $\}$ derecha $\}$

3. $\{$ dilatada la arteria cerebral $\}$ media derecha $\}$

4. $\{$ dilatada la arteria cerebral $\}\{$ media $\}$ derecha $\}$ 
5. $\{$ dilatada la arteria $\}$ cerebral media derecha $\}$

6. \{dilatada la arteria $\}$ cerebral media $\}$ derecha

7. $\{$ dilatada la arteria $\}$ cerebral $\}$ media derecha $\}$

8. $\{$ dilatada la arteria $\}$ cerebral $\}$ media $\}$ derecha $\}$

9. $\{$ dilatada $\}$ arteria cerebral media derecha $\}$

10. $\{$ dilatada $\}$ arteria cerebral media $\}$ derecha $\}$

11. $\{$ dilatada $\}$ arteria cerebral $\}$ \{media derecha $\}$

12. $\{$ dilatada $\}$ arteria cerebral $\}\{$ media $\}$ derecha $\}$

13. $\{$ dilatada $\}$ arteria $\}$ cerebral media derecha $\}$

14. $\{$ dilatada $\}$ arteria $\}$ cerebral media $\}$ derecha $\}$

15. $\{$ dilatada $\}\{$ arteria $\}\{$ cerebral $\}\{$ media derecha $\}$

16. $\{$ dilatada $\}$ arteria $\}$ cerebral $\}$ media $\}$ derecha $\}$

The subtrees (or potential entities) have to be matched with UMLS or the drugs database in order of relevance for the process to correctly annotate them as entities. Each annotated entity is given the unique identifier gathered from the source, being the CUI in the case of UMLS concepts. In the example given, the only possible annotations matching from UMLS are:

- $\operatorname{arteria}$ (CUI: C1285085)

- cerebral (CUI: C0228174)

- media (CUI: C0677540)

- arteria cerebral (CUI: C1278936)

- arteria cerebral media (CUI: C0149566)

- arteria cerebral media derecha (CUI: C1267298)

Note that the possible entities do not start with determiners (which are removed at the beginning of a subtree), to be able to match completely with the indexed terms. The subtrees are evaluated to match the biggest number of words in a single entity, to gather as much relevant semantic information together as possible.

In this example, the output generated in this process corresponds to the option number 9, which annotates arteria cerebral media derecha with CUI: C1267298.

This methodology is employed to determine which entities are annotated in a text, being annotated in this process both UMLS concepts and drug prescriptions. The process ensures that all the possible entities in a sentence are annotated by returning the best possible annotations. 


\section{Ranking the results to be matched from the sources}

In order to determine if the possible subtrees (potential entities) are entities extracted from the trusted sources, a search in them has to be made. In this particular scenario, given that the text to be searched can contain typos and that the search is performed on text, full-text search engines offer the necessary functionality to solve these issues.

In this particular method, and in order to integrate the search of concepts and their annotations with the rest of the pieces developed as part of this thesis, a REST Application Programming Interface (API) has been developed using Elasticsearch [Banon, 2011] as a backend search engine.

This REST API has been developed following the standards of these types of APIs to store the information from UMLS and from the drugs database for the annotation process. In what follows, an explanation of the ranking system performed when searching an entity is made.

In the case of UMLS concepts, the API retrieves a list of elements which are gathered around a base term (same string, different CUIs and SUIs). These elements, called tuples, contain the information of an UMLS concept with its CUI, the language, its SUI, the term and an id (a composition of the SUI and CUI).

In order to match the elements detected as possible entities to the results returned from the API, a ranking system has been developed. This evaluation is critical to the search process, as the API has to retrieve concepts in the UMLS and drug indices ordered with the most similar to the concept searched on top. The pseudocode for the ponderation of the elements can be seen in Listings 3.2, 3.3, 3.4, and 3.5.

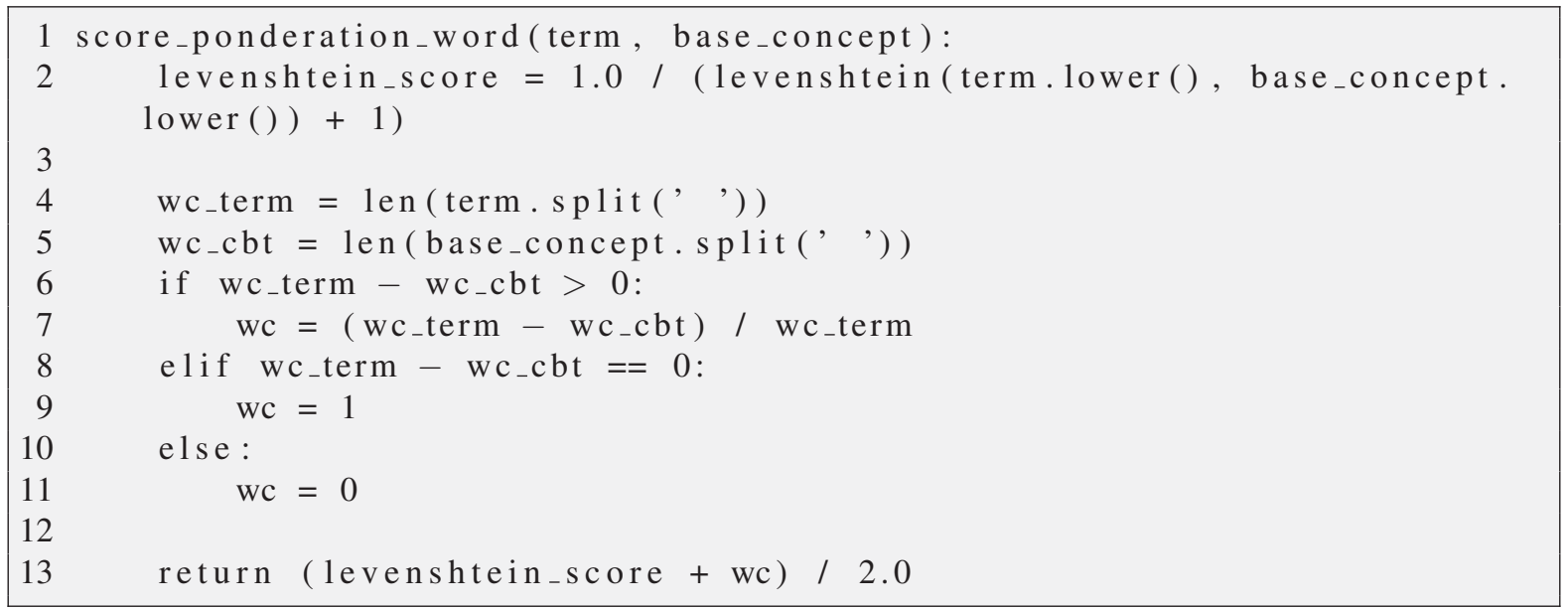

Listing 3.2: Pseudocode of the ponderation of words

Listing 3.2 shows a function in which a word is given a particular weight when compared to another word, which is used as a base of the concept (the base term is the normalization of different terms in a common, standardized term for the same concepts). Line 2 defines the inverse Levenshtein distance of the two terms in lowercase. The lower distance the better as the terms are more similar. Lines 4-11 define a simple whitespace tokenization to calculate the tokens in the two terms and the difference in the number of tokens, normalizing the result 
Chapter 3. Addressing specific challenges in the application of Natural Language Processing for Spanish Electronic Health Records

3.3. Detection of UMLS entities and drug names

between 0 and 1 taking the number of tokens of the desired term as the base. Line 13 defines the return value of the function as the average between this value and the Levenshtein distance.



Listing 3.3: Pseudocode of the ponderation of the language

Listing 3.3 defines the algorithm followed to assign weight to the results depending on their language. As the treatment is done with Spanish EHRs, Spanish is given preference. A list of terms is passed to this function (the tuples found on a result), and if the language is Spanish, 1 point is scored, and 0.5 points are given to the English results. The result of this function is the normalization between 0 and 1 of the ponderation given.

1 score_ponderation ( $r$, max_score ):

2 return $r$ ['_score'] / max_score if $\max _{-}$score $>0$ else 0

Listing 3.4: Pseudocode of the ponderation of the score given by Elasticsearch

Listing 3.4 shows the ponderation done with the score given by Elasticsearch. The score of each result is normalized between 0 and 1, being always 1 the one on top (the best result according to Elasticsearch evaluation).



Listing 3.5: Pseudocode of the ponderation for ranking the results

Listing 3.5 shows how the ponderation of the concepts is done. Three different scores are calculated by weighing the words searched and the ones returned in each result, the language in which each result is written, and the normalized score from Elasticsearch.

The result of this function, and hence, the final score given to each of the results returned, is the average of the three values. If there is an exact match, meaning that the result on top is the same as the one searched, and it is in the Spanish UMLS dictionary, the score given is 1. 


\section{Ranking and normalizing drug prescriptions}

Separate indices have been created with the information gathered from AEMPS [de Medicamentos y Productos Sanitarios, 2013]. The information provided consists on XML files containing information about prescriptions, commercial names of drugs, excipients, active principles, drug interactions, laboratories, packaging, and much more information about the drugs sold in Spain. The XML files contain 23,725 commercial prescriptions, 503 different excipients and 2187 active principles.

Any commercial drug is composed of excipients, which are commonly used in the jargon doctors use. Therefore, their appearance as part of the EHRs is quite common and they are used to state what type of treatment a patient is taking. Active principles are part of the components of drugs, but they are not as used as the excipients, but their appearance rate is high enough in EHRs to take them into account. However, prescriptions are one of the most widely used forms of drug mentions in EHRs. These are typically the commercial forms of drugs which in the end are the ones taking patients from the drug stores.

In what follows, an explanation of the scoring system excipients, active principles and prescriptions is presented.

First of all, information stored in the Elasticsearch indices has been normalized, to increase the precision when looking for a particular drug name. As an example of the normalization, we have many different prescriptions for Ibuprofen (ibuprofeno in Spanish). The API has some different examples of prescriptions for this drug name: I) IBUPROFENO DAVUR $20 \mathrm{mg} /$ ml SUSPENSIÓN ORAL EFG , 1 frasco de 200 ml; II) IBUPROFENO PENSA 600 mg COMPRIMIDOS RECUBIERTOS CON PELICULA EFG , 40 comprimidos; III) NORVECTAN 600 POLVO PARA SUSPENSION ORAL, 20 sobres.

In order to obtain better precision and be able to detect which prescription is being mentioned, due to its complexity, a base term of ibuprofeno has been indexed as the record to be returned when looking for that particular drug name. Under this base term, all of the different presentations are joint in a multi-element tuple. The first two were cleaned before indexing to match the term ibuprofeno as the rest of terms gather information of excipient doses and so on. But even if the third example does not mention ibuprofen, it does contain ibuprofen in the excipient list of the prescription, so it is matched with a correct ibuprofen search, even if the drug commercial name is way different than the search term. This has been also done with excipients and active principles lists for each prescription, creating a broader index able to increase precision of searches.

Before doing normalization the original database contains 23,725 commercial prescriptions, but after normalization, a total of 7,598 base terms are stored, with all the commercial prescription inside the aforementioned tuples.

The scoring system for prescriptions (see Listing 3.6), calculates the ponderation of the term searched compared against the base term indexed (line 2), and calculates the ponderation between 0 and 1 of the score returned by Elasticsearch (beign 1 the top result for the Elasticsearch index). The final score (line 5), is calculated as the average value of both individual 
scores. The auxiliary functions are the same as explained in Listing 3.2 and 3.4.

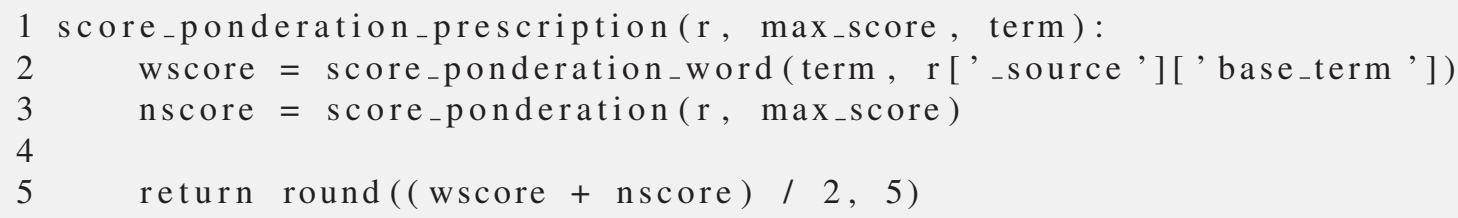

Listing 3.6: Pseudocode of the ponderation of prescriptions

\subsection{Detecting medical acronyms}

\subsubsection{The problem}

In the analysis of EHRs, one of the most challenging and relevant problems is the NER of such entities which are written as acronyms or abbreviations. The introduction of acronyms and abbreviations in the writing process to describe symptoms, procedures, and other conditions, inserts a new problem when re-reading the text and more importantly, when analyzing it by automatic processes. This typical behavior can lead to failures in the treatment of the patient if the proper meaning cannot be properly understood.

An acronym or abbreviation may not be descriptive enough, specially in those cases where the context does not allow to properly expand the acronym. This creates an ambiguity problem which, sometimes, it is also difficult for humans to solve.

The challenges behind acronym use and expansion depending on the context have been previously identified [Rodríguez-González et al., 2015]. Acronyms typically contain possible expansions and meanings, and therefore the context is often helpful. Moreover, current NLP systems only deal with detection of acronyms in their analysis of the text when the underlying vocabulary contains these acronyms. This problem increases with those acronyms that have several meanings within the same sentence or context. In this scenario, the disambiguation of the acronym is a challenge that remains an open problem.

The disambiguation of the acronyms and abbreviations, as well as the application of NLP in the medical domain, have been a recurrent topic in research in the last years, with different kinds of outcomes. In this section, the focus is on the main results produced in the area of acronym disambiguation in medical documents.

\subsubsection{State of the art}

Analyzing the literature many examples arise which are based on the automatic extraction of concepts, the detection of possible acronyms and the disambiguation of acronym-meaning pairs. The typical approach to this problem is to acquire a dictionary of acronyms and their possible meanings, and using NLP techniques to detect them in the text, to later on proceed with the disambiguation. 
In [Wren et al., 2002] an approach based on heuristics is presented. In this work, location and identification of acronym-meaning pairs have been done using subsets of MedLine records, obtaining more than 174,000 unique acronyms and 737,000 associated definitions. Pustejovksy et al. developed Acromed, a similar system to detect acronym-meaning pairs from abstracts stored in MedLine [Pustejovsky et al., 2001]. More complex approaches have been followed in [Feng et al., 2009] where the automatic extraction and acronym disambiguation in largescale organizational web pages was presented. In their study, the authors introduce two novel algorithms: an unsupervised ranking algorithm to filter out the incorrect acronym-expansion pairs and a graph-based algorithm to disambiguate ambiguous acronyms.

In [Melton et al., 2010], the authors mention that "several groups have generated sense inventories of acronym long form expansions from the biomedical literature. Long form sense inventories, however, may contain conceptually redundant expansions that negatively affect their quality". In the approach followed, a method to improve sense inventories has been developed. In this sense, their approach maps long form expansions to concepts in UMLS.

Other works have focused on the disambiguation of different terms found in the texts. One of the main papers in the field of acronym disambiguation for medical documents was developed by Sergei Pakhomov [Pakhomov, 2002]. The paper explains a method in which a semi-supervised maximum entropy based approach for the normalization of abbreviations and acronyms in medical texts is applied. Another work from Pakhomov [Pakhomov et al., 2005] introduces the disambiguation of acronyms and abbreviations in clinical narratives. In that experimentation, Google API was used in order to collect contextual data of 8 acronyms from EHRs at Mayo Clinic. A manually annotated corpus is used to disambiguate the meanings of the abbreviations found.

Machine learning approaches have also been tested as a methodology for the disambiguation of acronyms in [Zahariev, 2004]. Search engine queries were proposed to download documents to serve as training data, obtaining an accuracy of $92.58 \%$ in the identification of 47 acronyms on 9,963 documents.

In [Joshi et al., 2006b], Joshi et al. introduced word sense disambiguation and acronym expansion based on three different kernel methods: one using knowledge derived from unlabeled text, another one using ontologies to acquire the semantic knowledge, and the latest using additive kernel contained in the first two. Further studies shown in [Joshi et al., 2006a], reveal a comparative study of supervised acronym disambiguation on EHRs using three different machine learning algorithms with more than $90 \%$ of accuracy.

Acronym expansion using semantic networks is introduced in [Jeong et al., 2011]. In this approach, a semantic network is created by the merge of two heterogeneous resources linking information and measuring string similarity.

Expected Mutual Information Measure (EMIM) is used in [Limsopatham et al., 2011] between a long-form description of a concept and its corresponding abbreviation. This approach is used in order to measure the ambiguity of the term. A knowledge-based method to disambiguate acronyms by using second-order co-occurrence vectors with UMLS and MedLine concepts is introduced in [McInnes et al., 2011]. The approach, validated with 18 acronyms, achieves an 
accuracy of $89 \%$.

More recently, [Xu et al., 2012] propose the use of sense inventories in a two-phase clustering algorithm to detect rare senses of abbreviations in clinical notes. In [Li et al., 2015], Li et al. propose a two word embedding based-model for acronym disambiguation. The authors of this paper claim that their models outperform the state of the art methods on accuracy, showing that these kind of methods help to improve acronym disambiguation.

Research shows that two different approaches have been followed in this field: On the one hand, focusing on the automatic extraction of acronym-meaning pairs; On the other hand, on the disambiguation of the different meanings given an acronym and its context. Most significant approaches have been knowledge-based rather than machine learning focused, but, more importantly, none of the main efforts have been focused in the disambiguation of acronyms in Spanish EHRs.

\subsubsection{Solution}

Acronym disambiguation can be an endless process depending on the number of acronyms and the dictionaries used to give the acronyms their corresponding meanings. In order to perform disambiguation over the different acronyms found on an EHR, the following method is proposed to solve a particular problem in this area.

The method developed consists of 6 different steps:

1. From a given corpus to be used in the experimentation process, a high-rank selection of the documents with more number of acronyms is done. This approach has been selected in order to obtain a dataset considering the maximum number of possible acronyms in the corpus.

2. The selected documents are then cleaned up and manually annotated, by identifying the acronyms present in the text. Each acronym is then matched with all its possible meanings and the correct one is marked.

3. The selected dataset is then split into train and test sets.

4. The documents are then passed through the NLP pipeline developed in this thesis in order to obtain relevant information for the process, such as the section a document belongs to, and the PoS for each token.

5. Finally, the features to be used in the training process are extracted. These features were manually selected as it is illustrated in Table 3.8.

6. The documents are then transformed to CSV/ARFF files identifying in each line the acronym-meaning pairs together with context features.

In this section, an analysis over the disambiguation of medical related acronyms appearing in stoke patients EHRs by using the acronym-meaning pairs gathered from Sociedad 
Española de Documentación Médica (SEDOM) [de Documentación Médica (SEDOM), 2011]. In this approach, an experiment using C4.5 decision trees, implemented in WEKA [Hall et al., 2009], has been designed in order to create the models. The following subsections detail the dataset used and the performed experimentation.

\begin{tabular}{|l|l|l|}
\hline Feature & Description & Words in the example \\
\hline $\begin{array}{l}\text { pre-word, post- } \\
\text { word }\end{array}$ & Words before and after the acronym. & proximal - conservada \\
\hline acronym & The acronym. & MII \\
\hline area & Area of report. Not used. Future feature. & N/A \\
\hline definition & The definition obtained from SEDOM. & Miembros inferiores \\
\hline section & $\begin{array}{l}\text { The section of the report where the acronym } \\
\text { appears. }\end{array}$ & Exploración \\
\hline $\begin{array}{l}\text { PoS pre-word, } \\
\text { PoS post-word }\end{array}$ & $\begin{array}{l}\text { Part-of-Speech (PoS) of the word before and } \\
\text { after the acronym. }\end{array}$ & ADJ - ADJ \\
\hline label & $\begin{array}{l}\text { True/False, if definition is correct in that en- } \\
\text { vironment. }\end{array}$ & True \\
\hline
\end{tabular}

Table 3.8: Features used to train the model. Example sentence: "Fuerza proximal MII conservada y simétrica"

\section{Dataset}

A corpus has been collected with a total of 150 clinical notes. This dataset has been split into two different datasets: D1 and D2.

Dataset D1 - baseline Contains 6 features: pre-word, post-word, acronym, area, definition, and label. 9,627 instances exist after removing duplicates.

Dataset D2 Three more features have been introduced: PoS pre-word, PoS post-word, and the section in which the acronym is found within a document. We get 16,635 instances after removing duplicates. PoS helps to generalize the use of a meaning, as the same tags surround same meanings for a particular acronym, while others change the PoS of the surrounding tags. The section is an important factor, as the meaning of an acronym typically means the same within the same section.

- Source: EHR from a public hospital

- Type of documents: EHR and clinical reports

- \# of documents: 150

- Version: 1.0

- Availability: Proprietary due to confidentiality. 
Chapter 3. Addressing specific challenges in the application of Natural Language Processing for Spanish

Electronic Health Records

3.4. Detecting medical acronyms

\section{Experimentation}

In each of the datasets used (D1 and D2) there are a total of 7,148 acronym appearances with 480 unique acronyms. The acronym with a higher number of appearances is "U" (meaning "units" in most of the cases) with a total of 467 (6.53\%). Confusion matrices have been calculated to extract precision, recall and F1-score and to compare the results obtained in the different methods.

Two validation types have been performed:

\section{A 10 -fold CV}

2. A test-set from a 30 clinical notes subset randomly selected not used in the training phase.

For the test-set experiment, a 30 randomly selected EHRs set has been used, validating our approach using a realistic subset. The validation dataset contains 2,937 instances and 875 acronyms.

Confusion matrices in Table 3.9 depict the values for performance of the algorithm C4.5 using CV and test-set validation methods.

\begin{tabular}{|l|l|l|l|l|l|l|l|l|l|}
\hline \multicolumn{2}{|c|}{} & \multicolumn{2}{|l|}{$\begin{array}{l}\text { Classification } \\
\text { D1 - CV }\end{array}$} & \multicolumn{2}{l|}{$\begin{array}{l}\text { Classification } \\
\text { D2 - CV }\end{array}$} & \multicolumn{2}{l|}{$\begin{array}{l}\text { Classification } \\
\text { D1 - Test- } \\
\text { Set }\end{array}$} & \multicolumn{2}{l|}{$\begin{array}{l}\text { Classification } \\
\text { D2 - Test- } \\
\text { Set }\end{array}$} \\
\cline { 3 - 11 } C4.5 & & False & True & False & True & False & True & False & True \\
\hline \multirow{2}{*}{ Expert annotation } & False & 5935 & 249 & 10654 & 383 & 2082 & 315 & 2051 & 346 \\
\cline { 2 - 10 } & True & 404 & 3039 & 451 & 5147 & 49 & 490 & 45 & 494 \\
\hline
\end{tabular}

Table 3.9: C4.5 Confusion Matrices

From the confusion matrices in Table 3.9, precision, recall, and F1-score can be calculated, summarizing the results of $\mathrm{CV}$. Table 3.10 shows the results using these metrics.

\begin{tabular}{|l|l|l|l|l|l|l|}
\hline & \multicolumn{3}{|l|}{ C4.5 - CV } & \multicolumn{3}{l|}{ C4.5 Test-Set } \\
\cline { 2 - 7 } & Precision & Recall & F1 & Precision & Recall & F1 \\
\hline D1 & 0.932 & 0.932 & 0.932 & 0.909 & 0.876 & 0.885 \\
\hline D2 & 0.950 & 0.950 & 0.950 & 0.907 & 0.867 & 0.877 \\
\hline
\end{tabular}

Table 3.10: C4.5 performance metrics

Both models obtained a precision over $90 \%$, in line to the numbers shown in the state of the art for English language. Confusion matrices (see table 3.9) show that results of test-set have around $-8 \%$ F-score in comparison with cross-validation.

High-rank approaches tend to generate small biases as the most used acronyms have been used, so the most reasonable explanation is that such a bias was introduced. However, random selection of clinical notes does not ensure to correctly reduce the bias, though enabling a different test in a more "realistic" environment. 
The best model generated was using the CV method in the dataset D2, as it is the one with highest precision. In this particular experiment and scenario, precision is the most relevant metric. Nevertheless, in a "realistic" environment by performing the test-set, we can see that both models perform with similar patterns and the differences among them are not so relevant.

\subsection{Discussion}

In this chapter, some of the most relevant challenges in the application of NLP applied to EHRs have been addressed. Several contributions have been proposed in the analysis of negation detection, NER and acronym disambiguation. These contributions are:

- One of the first acknowledged negation detection approaches for Spanish written documents focused on the analysis of EHRs. The development of this approach and setting experiments on negation detection in Spanish following the example lead by English, German, and other languages, is an important milestone in this research.

Negation is a key factor specially in healthcare, as many of the entities mentioned are affected by some triggers imposing negation, hypothesis or uncertainty. The fact of being one of the first approaches developed, has been studied in the last years by other approaches that, nowadays, have improved the performance of the approach with the use of NegEx, ConText and other developed means. As a first approach, the results could have been improved by reimplementing new approaches on this matter, but an approach to replicate other studies was followed.

- A methodology to extract medical information. This framework, developed with an API, allows researchers to access medical information stored in well-known ontologies, like UMLS, with and standardized form in which the returned results are pondered so the match with the search terms is as equal as possible. This methodology proposes a solution to maximize the number and size of recognized entities while giving as much semantic information as possible.

The extension of this API with access to a complete dictionary of drugs sold in Spain, gives it a broader use case to work with. The standardization of this drug database helps physicians to look for specific drugs within EHRs independently on how it is written, if on the commercial form, the name of the excipients or the active principles involved. Therefore, this API can also be useful to automatically suggest commercial drug names of those prescriptions that doctors want to give their patients, by knowing its excipients or generic forms.

This API allows searching with fuzziness, enabling the systems using it to automatically detect relevant medical entities even if they are not properly written or contain some typos.

- An approach to detect and disambiguate acronyms. Acronyms play a big role in EHRs free-text as doctors tend to write in a timely manner and they typically use a lot of 
acronyms and abbreviations to express diseases, signs, symptoms or procedures. This extensive use of acronyms lead, many times, to misconceptions and it is more prone to unwanted errors.

In the approach presented, the detection of acronyms is done with an external dictionary of acronyms and abbreviations in Spanish. Noun Phrases are matched with those potential acronyms and the set of potential meanings is assigned. Different machine learning approaches have been followed in order to determine the correct meaning depending on the context of each acronym for the dataset used.

The contributions presented show that there are solutions proposed to solve the specific challenges in this sector. These methodologies and approaches have led to the completion of Objective O2, demonstrating that these problems needed to be addressed and solved.

The results of the research performed have produced the following publications:

- Roberto Costumero, Federico Lopez, Consuelo Gonzalo-Martín, Marta Millan, and Ernestina Menasalvas. An approach to detect negation on medical documents in Spanish. In International Conference on Brain Informatics and Health, pages 366-375. Springer International Publishing, 2014. DOI: 10.1007/978-3-319-09891-3_34

- Alejandro Rodríguez-González, Marcos Martínez-Romero, Roberto Costumero, Mark D Wilkinson, and Ernestina Menasalvas-Ruiz. Diagnostic knowledge extraction from MedlinePlus: An application for infectious diseases. In 9th International Conference on Practical Applications of Computational Biology and Bioinformatics, pages 79-87. Springer International Publishing, 2015. DOI: 10.1007/978-3-319-19776-0_9

- Ernestina Menasalvas, Alejandro Rodriguez-Gonzalez, Roberto Costumero, Hector Ambit, and Consuelo Gonzalo. Clinical narrative analytics challenges. In International Joint Conference on Rough Sets, pages 23-32. Springer International Publishing, 2016. DOI: 10.1007/978-3-319-47160-0_2

- Ignacio Rubio-López, Roberto Costumero, Héctor Ambit, Consuelo Gonzalo-Martín, Ernestina Menasalvas, and Alejandro Rodriguez-Gonzalez. Acronym disambiguation in Spanish Electronic Health narratives using machine learning techniques. Studies in health technology and informatics, pages 251, 255. IOS Press, 2017. DOI: 10.3233/978$1-61499-753-5-251$

- Alejandro Rodríguez-González, Roberto Costumero, Marcos Martinez-Romero, Mark D.Wilkinson and Ernestina Menasalvas-Ruiz. Extracting Diagnostic Knowledge from MedLine Plus: a Comparison between MetaMap and cTAKES Approaches. Current Bioinformatics. Accepted, yet to be published. 


\section{CHAPTER 4}

\section{The architecture - H2A: Human Health Analytics}

\subsection{Introduction}

The treatment of unstructured information in the healthcare sector is a huge challenge. The use of structured information in hospitals is scarce, mainly focused in personal information of the patient and laboratory tests, although most information generated in the EHRs is unstructured in the form of texts, images and graphs.

One of the problems in the medical domain is the extraction of knowledge from very complex textual datasets and the possibility to interpret them properly. But the real problem is not only to be able to analyze, understand and interpret the text written, but also to be able to create a complete system that is able to retrieve images based on the metadata and the annotations performed. An architecture that will serve as the basis for future systems to retrieve information from medical texts is then designed.

Focusing in the textual information gathered, the main source are the reports written by doctors after a consultation or a procedure, which are expressed in free-text. The use of NLP techniques could help doctors in the analysis of the textual information, rather than reviewing that information manually. Several studies have pointed the importance of the use of such techniques for clinical decision support to proceed with information retrieval, question answering and automated clinical trial recruitment [Tange et al., 1998]. These reports contain relevant information such as treatments, judgements, procedures that have been made, or the evolution of the patient. Rosenbloom et al. [Rosenbloom et al., 2011] shows the relevance of the automatic analysis of this information and how this information can be properly exploited.

The necessity of working towards solutions that are able to interpret Spanish written documents is also a problem, as many of the existing NLP processes are too focused for English texts. In the medical or other (very) specific domains, these kind of solutions are almost inexistent in many different languages. Therefore, there is a necessity in the design of a generic architecture for the NLP analysis that is able to interpret different languages (depending on the corpus used to train the models), and more importantly, that is generic enough to deal with many different types of documents and elements, has to ensure the accomplishment of the following characteristics: 
- Each component serves to solve a specific problem in NLP: Sentence boundary detection, Part-of-Speech tagging, parsing, entity recognition, etc.

- A common engine is able to create flows of the application that allow fixed or flexible executions of a pipeline.

- Configuration of the components in the pipeline is seamlessly integrated and this configuration is centralized, so changes performed for different purposes do not depend on coding new characteristics, but rather enable/disable the ones already implemented, working as switches of information to be analyzed.

- Allows the introduction of external resources to be used by the different components (databases, ontologies, etc.).

- The components are integrated in a common frontend to expose the analyzed information to external applications developed for the end-users.

The design of this architecture aims to fulfill the requirement of Objective O3, by creating a whole architecture that is able to analyze, interpret and manage unstructured data gathered from EHRs. The aim is to serve as a common architecture to be applied using documents written in any language and its NLP core is generic enough to be used in the analysis of documents gathered from different domains.

The rest of this chapter is organized as follows: Section 4.2 analyzes the state of the art of the projects and other architectures related with NLP applied to the EHRs, Section 4.3 describes the UIMA workflow used in the design of the architecture, and Section 4.4 shows the architecture developed.

\subsection{State of the art}

The information expressed in narrative form has been analyzed in different systems working on the NLP field applied to EHRs. One of this examples is the one developed in [Meystre et al., 2008] which analyzes the main challenges in the information extraction process from EHRs, as these documents typically contain shorthand phrases, abbreviations, acronyms, spelling errors and very specific nomenclature.

The increasing interest in the development of new NLP methods to be applied in the healthcare sector, and the growing use of EHRs have created a significant development of the so called Medical Language Processing (MLP) systems. Some of the projects have been described in [Friedman et al., 1994], [Zweigenbaum, 1994], [Friedman et al., 1995], [Friedman, 1997], [Fiszman et al., 1998], [Friedman et al., 1999], [Friedman, 2000], [Aronson, 2001], [Friedman et al., 2001], [Aronson, 2006] [Meystre et al., 2008], [Coden et al., 2009], and [Savova et al., 2010]. This systems are able to analyze different types of medical reports generated.

A text processing system was developed to analyze radiology reports to extract terms selected from a controlled vocabulary in [Friedman et al., 1994]. This system evolved to the 
MedLEE system introduced in [Friedman et al., 1995], which encodes clinical information found in the chest radiology reports that were evaluated. The MedLEE system has been extended to work on mammography reports and discharge summaries in [Friedman, 1997], and electrocardiography and pathology reports in [Friedman, 2000]. The same authors discussed on the relevance of NLP in medicine in [Friedman and Hripcsak, 1999], presented an use case of analyzing the severity of pneumonia cases in discharge summaries in [Friedman et al., 1999], and evaluated the performance of their system using different ontologies (LUMLS, M-CUR, M+UMLS).

Other systems regarding the use of NLP techniques alongside with machine learning, ontologies, bayesian networks, static rules, and other have been developed [Christensen et al., 2002], [Meystre et al., 2008], [Coden et al., 2009], [Savova et al., 2010], [Fette et al., 2012], [Doan et al., 2014]. MedTAS/P [Coden et al., 2009] introduces a system to automatically map concepts appearing in pathology reports into concepts represented by Cancer Disease Knowledge Representation Model (CDKRM) for scoring cancer characteristics and their relations. Health Information Text Extraction (HITEx) [i2b2, 2006] makes use of the Gate Framework to analyze different common problems such as the diagnoses extraction, discharge medication or the smoking status of patients. This system has also been used to extract the main diagnosis from 150 discharge summaries, including their co-morbidity and the smoking status in [Zeng et al., 2006]. Automatic knowledge acquisition is analyzed in [Taboada et al., 2013] by using a system reusing OpenNLP [OpenNLP, 2011], Stanford parsers, UMLS NormalizeString and other open-source tools, with the use of ontologies to enrich the documents.

In Fette et al. [Fette et al., 2012] an approach to extract information from medical texts is introduced. Based on Conditional Random Fields and keyword matching with terminologybased disambiguation this information is extracted from medical plain texts and integrated into a clinical data warehouse. Several clinical NLP systems are also described in [Doan et al., 2014], under a unified architectural view. Two main components are combined in this architecture: a background knowledge and a framework that include NLP tools. Christensen et al. [Christensen et al., 2002] introduce MPlus, a tool for analyzing medical text using Bayesian Networks (BNs). $\mathrm{BNs}$ are useful to represent semantic types and relations within a medical domain employing directed acyclic graphs whose nodes are concept types.

The use of NLP techniques has also been used to detect the overuse patterns of medication from patients by analyzing their EHRs [Salmasian et al., 2013]. It has also served to detect suicide or suicidal attempts based on the text written in EHRs [Haerian et al., 2012a], or to detect adverse events of drugs in pharmacovigilance processes [Haerian et al., 2012b].

One of the most popular NLP applications for analyzing English biomedical texts is MetaMap [Aronson, 2001], [Aronson, 2006]. MetaMap is a software developed to map medical terms found in biomedical reports to knowledge sources like MeSH [Lowe and Barnett, 1994] and UMLS [Humphreys and Lindberg, 1993]. It is able to parse the text and generate term variants, find synonyms and analyze abbreviations and acronyms. It is presented as a generic set of algorithms not only specific to the biomedical field, but also extendable to other domains with the appropriate knowledge sources.

Another of the most popular NLP applications that has been included in the Apache 
open-source catalog is cTAKES [Savova et al., 2010]. It is an open-source NLP system that uses rule-based and machine learning techniques to process and extract information to support clinical research. It is composed of several components such as sentence boundary detector, tokenizer, normalizers, part-of-speech (PoS) taggers, shallow parsers and NER annotators. It bases the NER in the use of ontologies such as UMLS. The development of cTAKES is based in the UIMA framework, so it creates a complete pipeline by describing annotators for specific purposes, like detecting the smoking status of a patient. However, one of its counterparts is the lack of supporting other languages different than English.

UIMA, created by David Ferrucci and his team at IBM back in 2004, is the "de facto" standard for the analysis of unstructured information, as its acronym stands for. This architecture, implemented mainly in Java, allows the creation of data structures that simulate the objects present in texts, images, voice records, etc. It has been used in many open-source projects like cTAKES [Savova et al., 2010] and internally in IBM in well-known systems such as IBM Watson [IBM, 2011].

There exists also commercial applications that envision the power of the use of NLP, machine learning, and many other techniques to benefit the healthcare sector, applying algorithms to extract knowledge from EHRs. The most renowned project is IBM Watson [IBM, 2011], a system developed by IBM that is able to answer to questions posed in natural language over an open domain. IBM has also created a healthcare focused version, called IBM Watson Health [IBM, 2014], an extension of the functionalities to use the same technology in helping doctors understand diseases and with the aim of expanding also the analysis to images and any kind of records held at hospitals.

Some NLP projects, systems and architectures to analyze documents in other languages have been created [Pietrzyk, 1991], [Zweigenbaum, 1994], [Hohnloser et al., 1996].

MENELAS [Zweigenbaum, 1994] processes patient discharge summaries to extract information form them. These reports can be written in French, English and Dutch, and MENELAS can take care of them. Medical Text Analysis System (MediTAS) [Pietrzyk, 1991] is a system able to parse summary sections of cytopathological findings reports an approach called LeftAssociative Grammar (LAG). For the German SNOMED II version another parser is presented in [Hohnloser et al., 1996] which is able to divide medical terms into fragments containing other SNOMED terms.

In this scenario of multilingual applications to analyze EHRs, up to our knowledge the only tool developed to analyze Spanish EHRs is [Savanamed, 2016] [Medrano et al., 2017]. The efforts in the development of this thesis to develop a system for Spanish EHRs try to fill this gap creating new approaches and systems.

\subsection{The UIMA workflow}

UIMA offers a set of frameworks to interact with by using C++ or Java, being the latest the one used in the implementation of the architecture. These frameworks offer an API to connect user 
applications to the UIMA engine in order to structure the unstructured data. But the definition of UIMA not only comprises of an implementation and some frameworks, but also is composed of some infrastructure (servers and other common tools for scaling the application), and of some core components, such as the annotators and type systems that are developed in the UIMA user space. For a quick hands-on on what UIMA offers, see Figure 4.1.

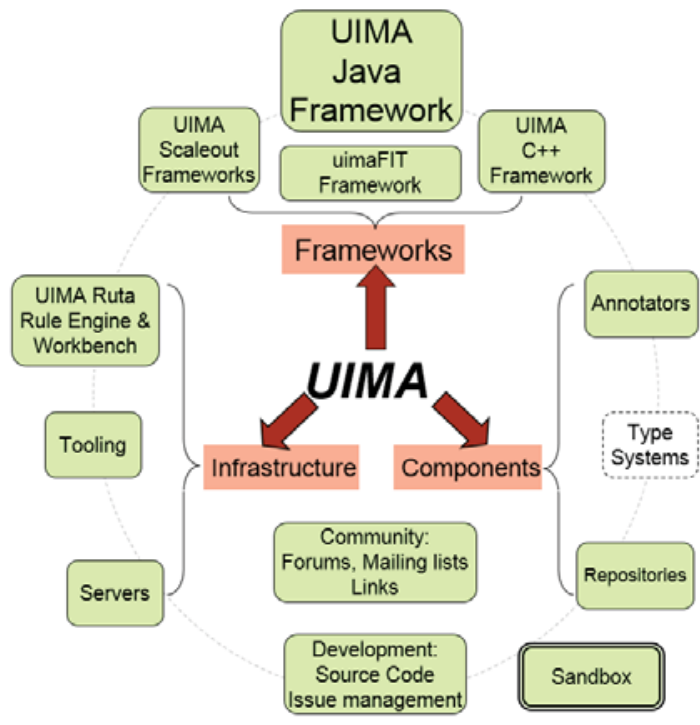

Figure 4.1: UIMA description of the components [Ferrucci and Lally, 2004]

When an application is built on top of UIMA, the definition of the objects is done by the developer, but the execution is done by the UIMA core engine. UIMA creates a set of annotations from the source documents to be analyzed based on the definitions made by the developer. These annotations are stored in the Common Analysis Structure (CAS), a data structure that contains the information to be annotated (the raw text in the case of analyzing textual documents), and indexes with the different annotations once the document has been processed.

The developer has to create several components in order to define the objects that will be annotated. An example of such object, can be the tokens defined in a sentence. These components to be developed are:

1. A Type System that defines the types of the different items to be annotated. Each annotation has a type system, which may have as many items as desired that can be native types (integers, float, strings) or data structures such as lists and arrays. An example of these items is the type of token (if it is a punctuation symbol, a word, etc.)

2. An Annotator, implemented as a class in the chosen programming language, that implements what has to be done in the annotation process. It contains a "process" method that is called upon its execution and contains the algorithms and the logic of the annotation.

3. A Collection Processing Engine (CPE) Descriptor, typically implemented in an XML file that defines which are the annotators to be run, the type systems to be used and the expected inputs and outputs. A descriptor can be a simple descriptor, in which only an 
annotator is run, or an aggregated one in which several annotators can be run in a singleflow pipeline or a parallel one.

Once the programmer has defined what and how to annotate the objects represented, the CPE Factory gathers the source documents, creates Analysis Engines to run the descriptors, and create the corresponding CASes. An overview of the UIMA architecture can be seen in Figure 4.2.

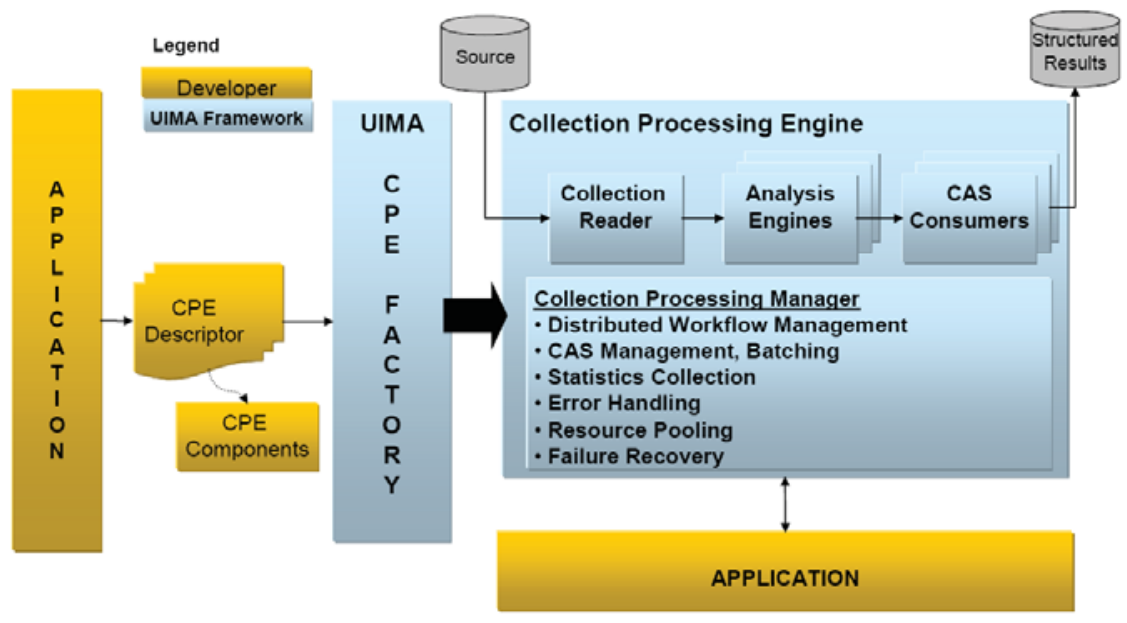

Figure 4.2: UIMA’s architecture [Ferrucci and Lally, 2004]

\subsection{H2A: Human Health Analytics - Architecture}

After reviewing the state of the art in the automatic analysis of EHRs, several already existing solutions came up as possible initial solutions in solving the problem: cTAKES, GATE or MetaMap to put some examples. The main problem with these frameworks is that they are, at the time of writing, too English-centric, meaning that most of the developments done are not suitable to use in other languages, or the possible modifications become too complex. They also perform only analysis of textual information and lack the inclusion of other unstructured data relevant like images gathered from the tests performed to the patients. In this sense, it is necessary to adopt more generic standards to build new frameworks allowing the definition of common structures independently on the language or process to be performed. H2A has been designed from scratch and has been developed on top of Apache UIMA framework to build a NLP framework as it fulfills the requirements. OASIS Consortium [Consortium et al., 1993], has also approved UIMA as an open standard for analysis of unstructured information ${ }^{1}$. Therefore, UIMA has been the framework selected to become the basis of the proposed solution.

Along the different chapters of this thesis, different challenges have been addressed and several problems have been solved. More concretely, a methodology to train models using

\footnotetext{
${ }^{1}$ https://www.oasis-open.org/standards
} 
generic and domain specific corpora has been done, solutions to detect negated entities, to detect medical concepts and drug names, and to disambiguate acronyms, have been developed. Each of these solutions have been developed as individual components to solve these problems, together with other less-relevant but necessary components.

The definition of a new architecture allows to bring those components to work together in a single environment to serve as the basis for the creation of future applications.

To understand the development of the new architecture, the improvements proposed to the typical NLP pipeline are detailed. Finally, the definition of the architecture and a possible implementation are presented.

\subsubsection{Improvements proposed to the typical Natural Language Processing pipeline}

The typical pipeline (see Figure 4.3) serves to the purpose of analyzing texts and extracting (basic) relevant information from any given text. However, some more complementary components should be added to the base version of any pipeline in order to perform complex text analytics in any given domain. Figure 4.3 was already explained in Section 2.3.1 and has been included here for better understanding on the improvements made.

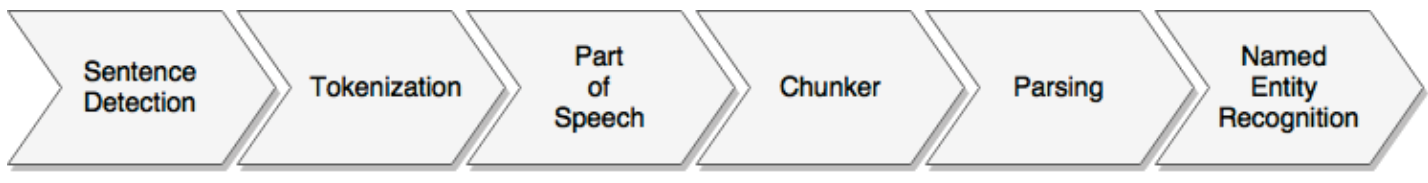

Figure 4.3: A typical NLP pipeline

The first modification proposed is to complement the division of the text in its basic structures, so it is easier to determine the context of a given entity. The new proposed pipeline (see Figure 4.4) will create a new structure instead of the sentence detection and tokenization, called "Text structure recognition", which is really the composition of these two modules in inverse order, with a newly created component that is able to determine the scope of paragraphs inside a text.

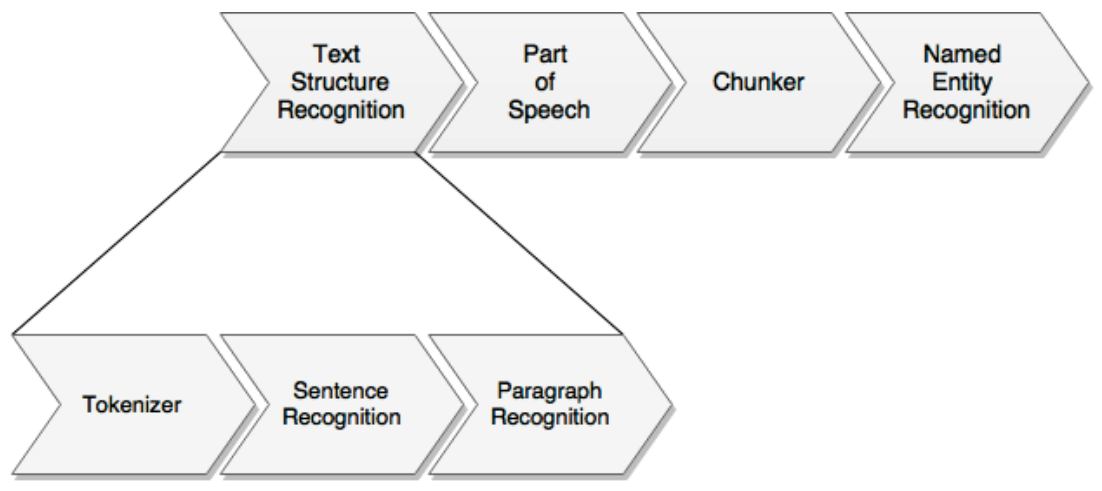

Figure 4.4: The newly proposed pipeline 
The change from the typical order - although not always followed - in the components of sentence detection and tokenization, gives a clearer view on the detection of the sentences, after getting a basic whitespace tokenization.

By adding the Paragraph Recognition component, a new relevant piece of information is added to the pipeline, which helps to contextualize the data that is gathered from the text. This component is really critical when analyzing entities in any document, as it helps to broaden the context not only to the same sentence, but also to the whole paragraph containing the entity.

The second change performed in the newly introduced pipeline, is the disaggregation of the Named Entity Recognition phase in several individual components, that help to create a modularized NER that is able to assign better features to the entities recognized in the Named Entity Recognition. The features added thanks to the components that can be executed, together or alongside the NER, can be used to determine different aspects of the entity, like getting the values in a laboratory test, or getting the metrics of a particular dose of a drug given to a patient.

These components added can be developed independently but added to the main flow of the pipeline, so the information can be reached when analyzing the entities. As it has been explained, the purpose of these components can vary depending on the particular problem to be tackled and, once again, work like a switch which is configurable at runtime. An example for these kind of modules attached for analyzing Electronic Health Records can be found in Figure 4.5.



Figure 4.5: An example of an extended Named Entity Recognition module

Finally, the third relevant change is the addition of the three components developed as part of the research introduced in previous chapters: The Medical Entity \& Drug Recognition, the Medical Acronym Recognition and the Negated Term Detection. These components are relevant for the medical domain.

The information extracted from these components is particularly relevant when performing text analytics, as the results of applying this kind of features to the analysis of a text, varies greatly when adding this information or not. The addition of this three contributions to the generic pipeline gives a new perspective to prepare text for performing analytics. These modifications and the newly created pipeline (see Figure 4.4) are the basis for the related projects developed. 


\subsubsection{Defining the architecture}

An architecture to be applied in EHRs has to take into account the changes proposed to the NLP pipeline, while adding specific components relevant for the healthcare sector. The creation of such generic architecture has opened up many opportunities in bringing the analysis of two different kinds of unstructured data: text and images. However, although the design of such generic architecture has been done in this thesis, the development of the different components have been focused in the NLP part, leaving the analysis of images and the actual integration alongside with the structured information for analysis as future work.

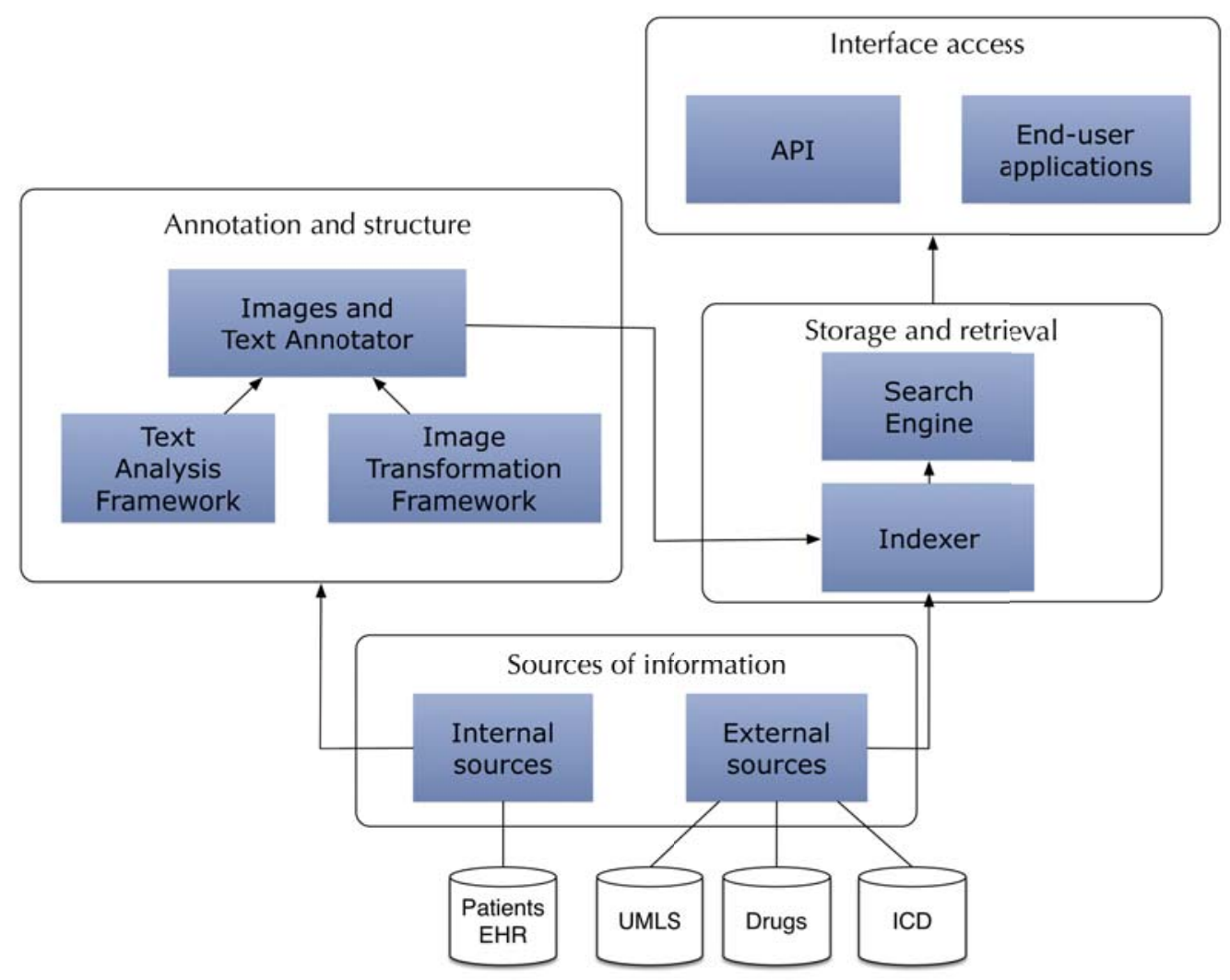

Figure 4.6: Architecture developed to analyze Electronic Health Records

The architecture consists of 4 different blocks (see Figure 4.6):

Sources of information The information is gathered from two main sources: Internal sources at the hospital level, being the EHRs collected from patients; and External sources, being the most widely used standards and open databases to help in the structuring process: UMLS, ICD, and some drug name databases, to name a few.

Annotation and structure The information gathered from the internal sources has to be structured. This information can be in form of text and images, so there is a framework for each of these types that is able to structure the relevant information contained on it. Both, the Text Analysis Framework and the Image Transformation Framework should output 
the same type of annotations in order to be able to combine both types of data. The Images and Text Annotator is capable of generating UIMA annotations and combine them on the different EHRs.

Storage and retrieval The annotations gathered from the previous process and all the external sources should be indexed in common indices in the Indexer component. This will store the structured information to serve data to the Search Engine responsible for the information retrieval.

Interface access Finally, an interface access has to be developed in order to serve the information for the end-users or external applications to consume the structured data to generate knowledge in different forms. This access is given as an API, for other applications to access the data, and as end-user applications for doctors to consume the annotated data.

This architecture has been named H2A: Human Health Analytics. H2A is a software architecture that has been designed to analyze and extract hidden knowledge in the patients Electronic Health Records.

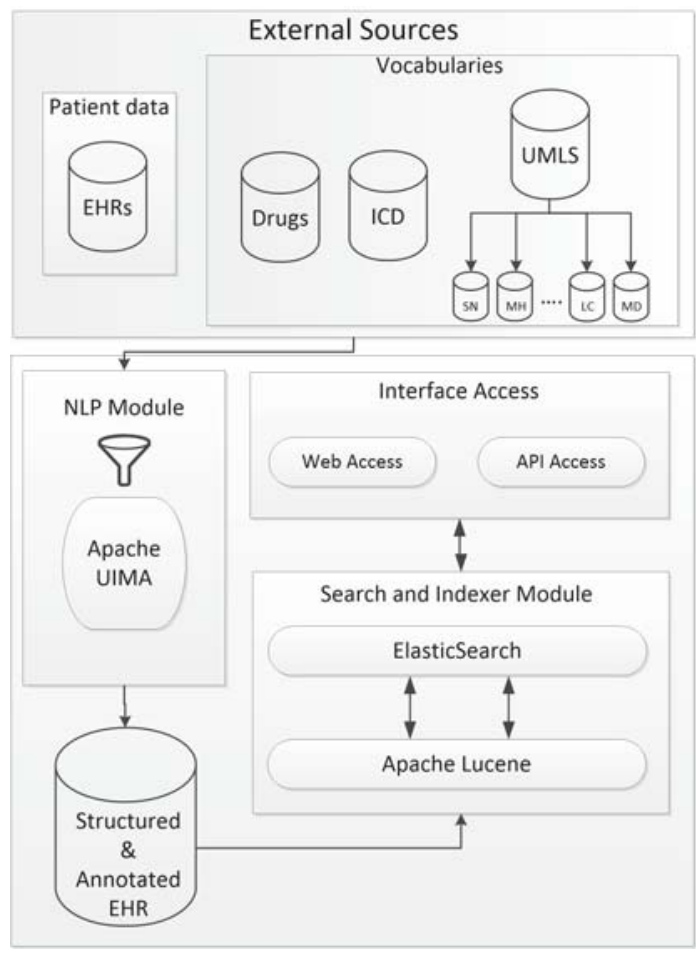

Figure 4.7: Text analysis framework architecture developed to structure the text written

Focusing on the Text Analysis Framework (see Figure 4.7), which is the aim of this research, several technologies have been proposed as the ones to be implementing this framework. As the main sources of information, UMLS and ICD coding standards have been chosen. UMLS gathers different sources of information and standardizes the codes used for representing the same information from these sources. Among these sources SNOMED-CT, LOINC or RxNORM, can be found. ICD has also been included as it is commonly used in Spanish 
hospitals. A drugs database has also been included with the information from the nomenclator gathered by the AEMPS [de Medicamentos y Productos Sanitarios, 2013].

For the NLP part in the Text Analysis Framework, OpenNLP [OpenNLP, 2011] has been chosen to train and deliver the statistical models for the language recognition, and the UIMA architecture has been used for deploying the NLP services in a common pipeline, following the standards in these types of applications. The NLP process structures the information in the EHRs that is going to be indexed. For this matter, Apache Lucene [The Apache Foundation, 2000] and Elasticsearch [Banon, 2011] have been chosen as the technologies to provide storage and full-text search to the information. Finally, a REST API and web applications can be developed to work with these technologies in order to deliver the knowledge extracted to the end-users. This piece of the architecture adds a complete structured relational database before feeding Lucene and Elasticsearch with data, in order to be able to have an structured source of data to perform complex analytics.

\subsubsection{Implementing the Text Analysis Framework}

The proposed implementation of the Text Analysis Framework has been done by using the Eclipse software [Eclipse, 2009], as main Integrated Development Environment (IDE) for development, using Java 8 as main programming language. Many other technologies have been used to help in the development of the architecture, such as: Maven, Git, and MySQL.

\section{The execution process}

The architecture follows a simple execution flow (see Figure 4.8) when generating its outcomes. In the implementation of the architecture there is a main process, called "H2A Process", which gets raw text documents as input. These are plain text versions of the EHRs, clinical notes, radiology reports, and any kind of medical textual document produced.

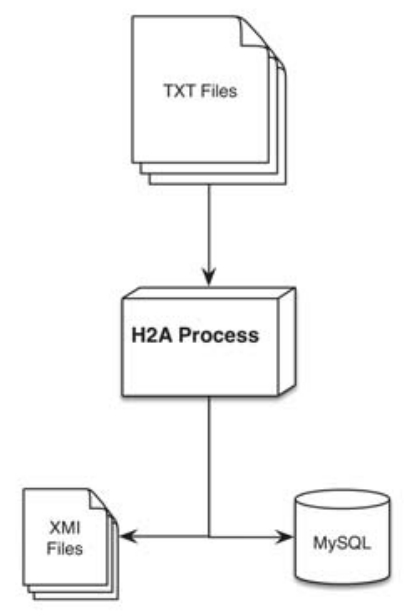

Figure 4.8: Generic inputs/outputs of the system 
When the main processes in $\mathrm{H} 2 \mathrm{~A}$ are executed, two resources are generated:

1. One XMI file for each of the processed documents, which is generated by the UIMA annotators. These documents contain all the existing annotations in the given text after being processed by each of the annotators. Each of this files contain structured information of the relevant information in the EHR.

2. A structured relational database hosted in a MySQL server that contains the information of the most important annotations. These annotations have been stored in a relational database to help with the process of performing analytics on the data, as a datastore and failsafe copy of the data, and to allow more flexibility than the use of the XMI files. The design of the database can be seen in Figure 4.9

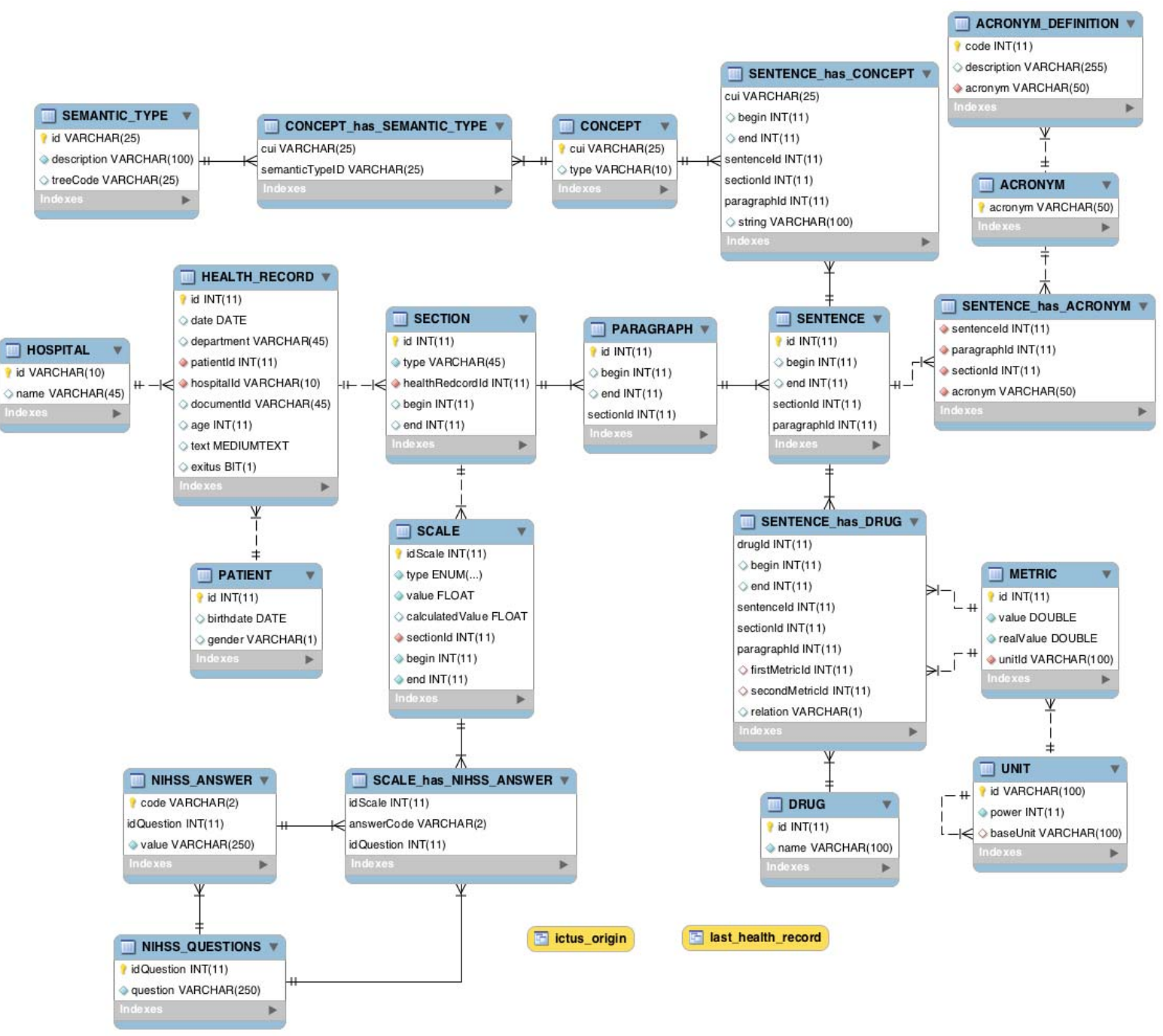

Figure 4.9: Design of $\mathrm{H} 2 \mathrm{~A}$ relational database which contains the most important annotations.

The design of the database has been done taking into account only the most important annotations that will be useful in future projects to develop analytics on any generic project 
using EHRs. In this database we store the EHR, its corresponding hospital and patient, its whole text, and the different sections of the text disaggregated. In order to achieve the maximum granularity possible, the text has been divided into sections, paragraphs and sentences, to allow understand the context of the different entities. Finally, UMLS concepts have been also added, alongside with the acronyms, drugs, metrics and the different medical scales found.

\section{Eclipse project dependencies}

A total of seven different projects (see Figure 4.10) have been developed in this framework, to ensure the modularity of the framework and the use of each one of them separately if needed in external projects. These projects are used by each other depending on their necessities, and they implement specific functionalities in the corresponding UIMA descriptors.



Figure 4.10: Project dependencies

utils Implements common data structures and connections to the database.

nlp-es-tokenizer Implements a whitespace tokenizer able to detect sentences, paragraphs and tokens.

nlp-es-general Provides a common interface to the generic NLP tasks, such as PoS tagging and Shallow Parsing.

nlp-es-metrics Gives annotation on metrics used, such as the drug doses.

neuro-scales Provides functionality to detect the different neurological scales that are mentioned in the EHRs.

h2a Implements the core of the architecture giving functionality on the health-related annotations, such as concepts in UMLS, acronyms or drug mentions.

h2a-process Contains the logic to execute the complete $\mathrm{H} 2 \mathrm{~A}$ pipeline, generate the XMI files, and load the database with the proper information. 


\section{Whitespace Tokenizer}

The inputs and outputs of the Whitespace Tokenizer are depicted in Figure 4.11. This type of tokenizer takes raw text files as an input and it outputs the corresponding XMI files generated by UIMA. This tokenizer has been developed to detect not only tokens, but also act as the Sentence Detector in the NLP process of the architecture. An extension, makes it possible to detect also paragraphs in the text, incrementing the possibilities of adjusting the scope for the entities mentioned.



Figure 4.11: Whitespace tokenizer pipeline

\section{Metrics descriptor}

The metrics descriptor, depicted in Figure 4.12 makes use of the whitespace tokenizer in order to detect different kinds of metrics. Three different annotators have been developed with the aim to annotate particular structures:

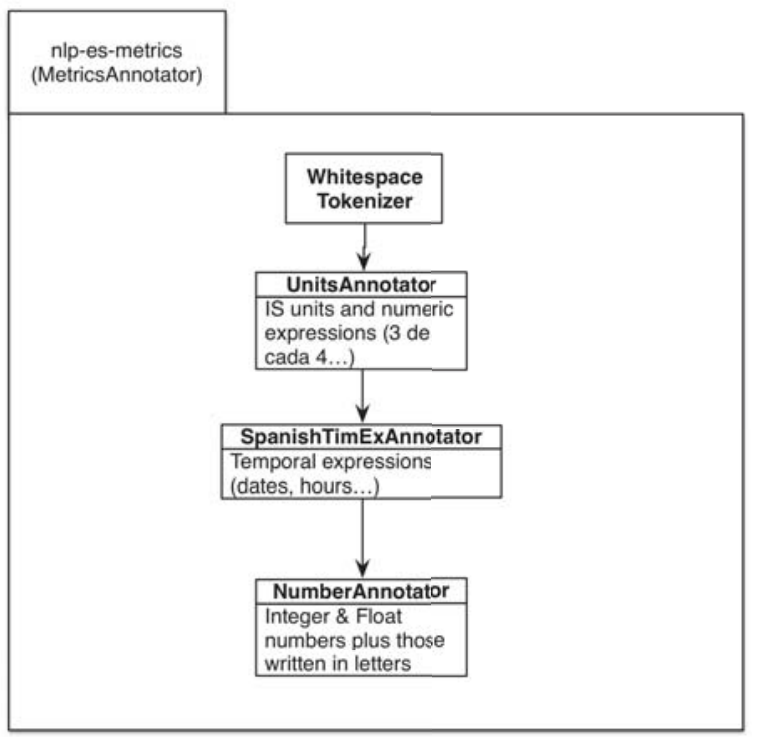

Figure 4.12: Metrics pipeline annotators 
Units annotator This annotator is capable to detect metrics written in the International System of Units and different numeric expressions.

SpanishTimEx annotator This annotator is build to detect temporal expressions based on the ones in [Saur1 et al., 2010].

Number annotator This annotator is capable of detecting numbers, classifying them as integers or floating point numbers, recognizing even the ones written in letters in Spanish.

\section{General NLP descriptor}

This descriptor implements the more general processes of NLP except the sentence detection which is done by the tokenizer. It makes use of the metrics descriptor in order to assign the proper values to the different tokens recognized as metrics, which otherwise could be mislead.

The general NLP descriptor (see Figure 4.13) makes use of Apache OpenNLP in order to execute the models that have been previously trained for the PoS tagger and the Chunker.

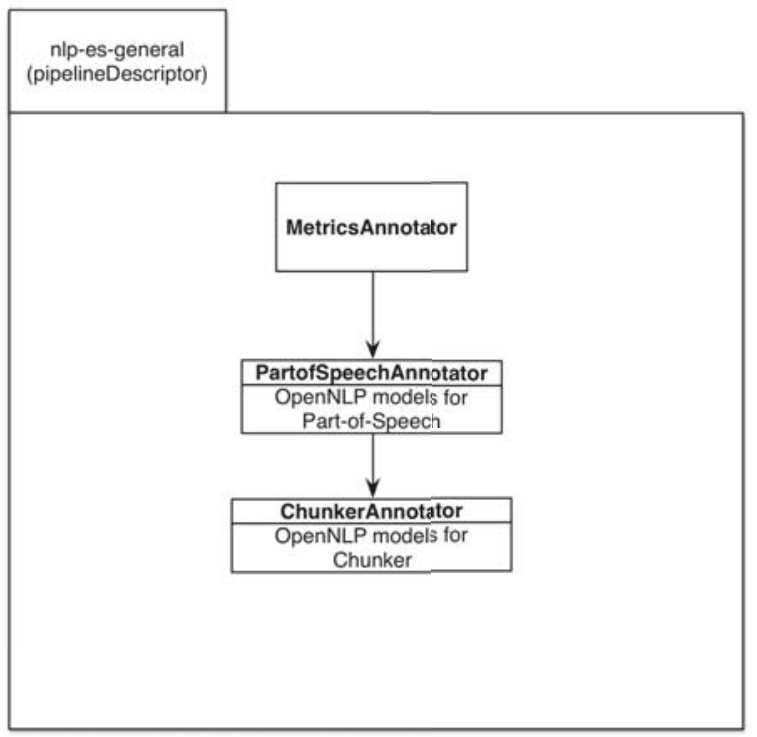

Figure 4.13: NLP pipeline annotators

\section{H2A pipeline}

H2A pipeline depicted in Figure 4.14 implements the general annotators to be applied in the healthcare domain. By making use of the NLP general pipeline, the annotations made as Noun Phrases, Adjectival Phrases and Prepositional Phrases are taken into account to search in the UMLS ontology for diseases, parts of the body, or other health-related entity contained on it. 
The same phrases are also used by the Acronym annotator which finds the potential acronyms and abbreviations and assigns them the possible meanings extracted from the SEDOM acronym dictionary.

The Record Section annotator allows to detect the different sections in the EHRs such as the history of the patient, evolution, exploration, tests performed, treatments and the follow-up.

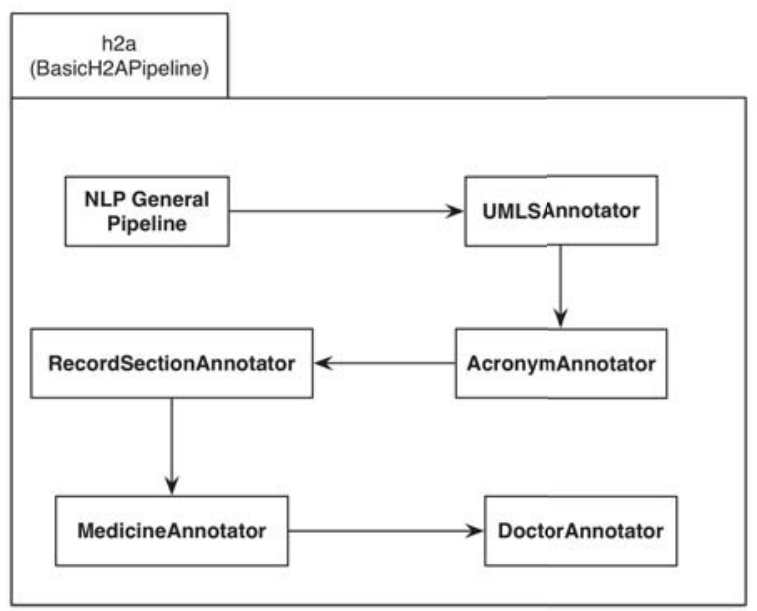

Figure 4.14: H2A pipeline annotators

The Medicine annotator is the responsible of annotating all the different drugs found in the text of EHRs. These drugs are annotated with the different formats in which it is sold, its excipients and information about the interaction with other drugs. The information used by this annotator is gathered from the nomenclator gathered by the AEMPS [de Medicamentos y Productos Sanitarios, 2013]. Finally, H2A pipeline is able to detect the doctors signing a clinical report included in the EHR.

\section{Providing functionality for end-user applications}



Figure 4.15: End-user application integration

The architecture offers functionality to develop and create end-user applications or API endpoints to access the data that has been structured. Figure 4.15 shows the schema of the created API and the connection of the data to the end-user applications. The information that 
has been stored in Elasticsearch indices is consumed by a Python REST API that allows the proper interaction of the end-user applications with the data.

\subsection{Discussion}

$\mathrm{H} 2 \mathrm{~A}$ architecture has been developed as part of this research to provide a generic architecture that structures and allows the analysis of texts written in EHRs.

One of the main problems in order to automatically analyze EHRs is the unstructured information that they contain, that has to be structured to give it some sense and extract knowledge from it. The architecture proposed includes the use of different databases and ontologies used in order to properly structure the information. It also includes the development of a NLP pipeline that analyzes in different aspects the text written in EHRs to annotate, using UIMA annotators, the different key entities described in the text.

The architecture has been designed to work mainly in the healthcare domain and analyze EHRs but, although the focus is put on this domain, most of the components developed have been designed as generic components that can work seamlessly in other domains or areas. A particular example of the application of H2A in the analysis of EHRs is shown in Chapter 5. The design of this architecture have led to the achievement of Objective O3.

The following publications introduced the architecture and the problems to be solved as part of the contributions on this research:

- Roberto Costumero, Angel Garcia-Pedrero, Isaac Sánchez, Consuelo Gonzalo, and Ernestina Menasalvas. Electronic Health Records Analytics: Natural language processing and image annotation. BIG DATA AND APPLICATIONS, page 1, 2014.

- Roberto Costumero, Consuelo Gonzalo, and Ernestina Menasalvas. TIDA: A Spanish EHR semantic search engine. In 8th International Conference on Practical Applications of Computational Biology \& Bioinformatics (PACBB 2014), pages 235-242. Springer, 2014. DOI: $10.1007 / 978-3-319-07581-5 \_28$ 


\section{CHAPTER

\section{Applying H2A: Human Health Analytics to analyze the evolution of stroke patients}

This chapter focuses on the application of $\mathrm{H} 2 \mathrm{~A}$ architecture to a specific use case using real data. In order to demonstrate the feasibility of the technology, an application has been developed to aid doctors in the analysis of stroke patients.

\subsection{Introduction}

Neurology doctors treat many patients suffering from strokes every week. Doctors and specialists in neurology have developed methods in order to evaluate the severity of stroke patients. Typically this methods involve an evaluation of the patient by answering questions on a standardized form which is filled and analyzed by the doctor. These methods involve different scales that indicate, in many cases, the neurological functionality of the patients. The values from the scales typically appear on the EHRs of the patients written in free text, what makes it only useful in the case of active revision from the doctor of those particular documents.

Doctors make a continuous evaluation of the evolution of these patients as they are typically taken care in Intensive Care Units (ICUs) due to the severity of this disease. Therefore, doctors need methods to assess the evolution of the patients based on objective data gathered from different tests.

When a patient is diagnosed from suffering a stroke, doctors use tests to assess the severity of the patients. In these tests the doctors ask questions to the patients and make observations on different aspects: mobility, coordination, speech, etc.

In the particular case introduced here, doctors from the stroke unit from a public hospital have gathered this information for years in order to evaluate the evolution of their patients and to address changes in their methodology if necessary. However, current information systems deployed at hospitals do not benefit from the automatic extraction of the information hidden in the clinical narrative. Typically, a doctor can gather information of particular documents or all the documents from a given patient, but the analysis of most parts of the information has to be done manually. In the case of stroke patients relevant information, such as the scales previously mentioned, is not automatically collected.

Doctors need from automatic tools to perform reporting on the patients and from interfaces to access the data gathered. $\mathrm{H} 2 \mathrm{~A}$ has been designed with a particular end-user application 
to access to the information gathered in these types of EHRs so doctors can evaluate their patients' information. This particular application of the research performed, shows how to improve the ontology based NER by using the information of the most used stroke scales in the EHRs, in particular: National Institute of Health Stroke Scale (NIHSS), Barthel Index (from now on: Barthel), Canadian Neurological Scale (from now on: Canadian) and Modified Rankin Scale (from now on: Rankin). This method annotates, extracts and analyzes discharge reports written in Spanish from patients suffering any kind of stroke history. Doctors are now able to use $\mathrm{H} 2 \mathrm{~A}$ to perform the analysis on the evolution of their patients.

The application developed helps doctors to use different criteria when searching for their patients, so an analysis on their status and their evolution can be made. The status and the complete reports of the patients are also available to perform more accurate analysis.

In the following sub-sections, an analysis on the background of the study, as well as the results of the application of $\mathrm{H} 2 \mathrm{~A}$ to the dataset given are explained in detail. To sum up, a discussion and future possible works of this particular application are presented.

\subsection{Background}

\subsubsection{Analysis on strokes}

A stroke, also known as Cerebrovascular Accident (CVA), Cerebrovascular Insult (CVI) or brain attack, is a disease that affects blood circulation on the brain leading to different outcomes, including death. It is one of the most deadly diseases nowadays, and is one of the diseases with quicker evolution due to ageing population. In Spain, average life expectancy is 82.4.

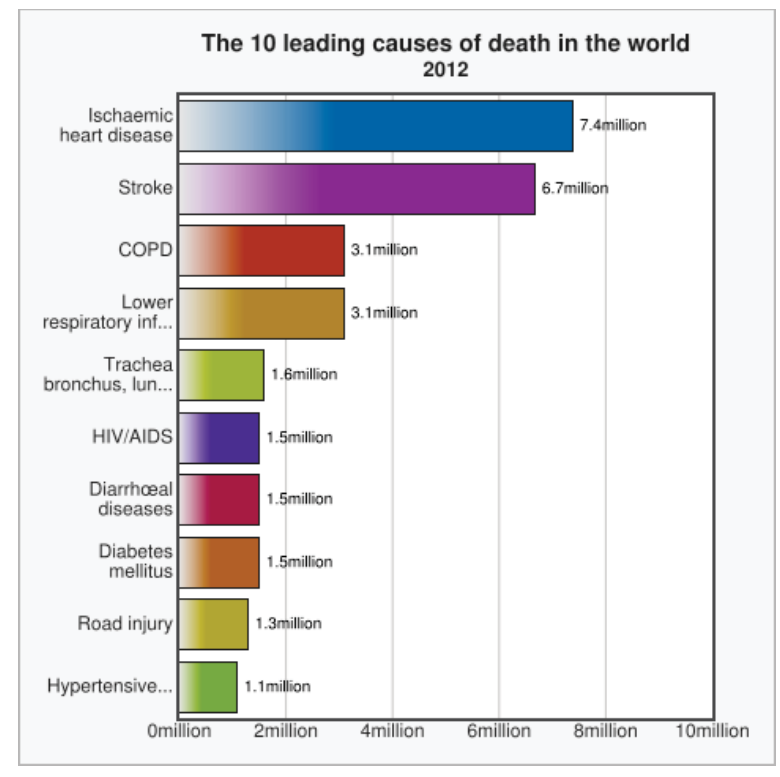

Figure 5.1: Mortality rate [Organization et al., 2014]

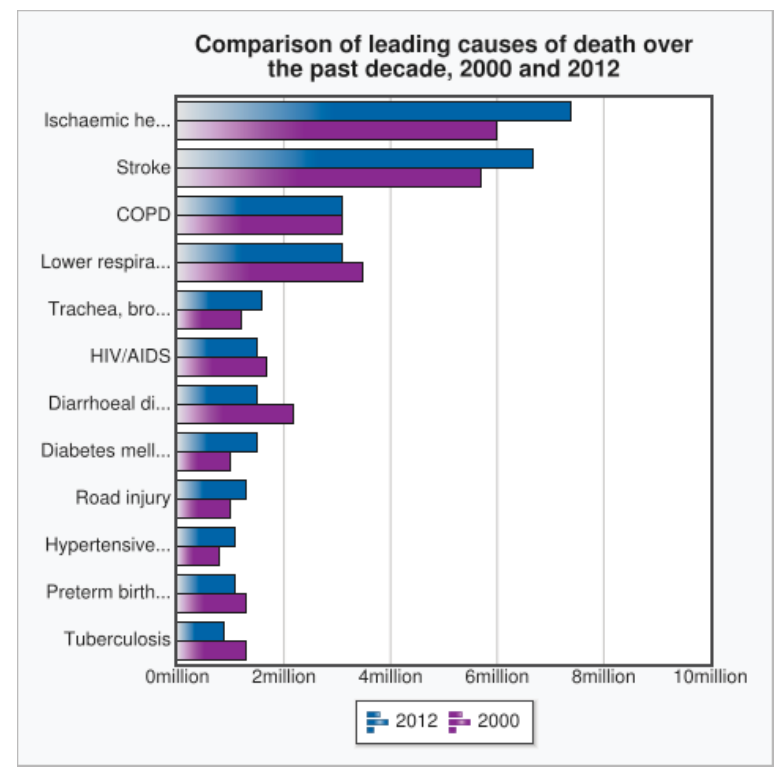

Figure 5.2: Mortality rate [Organization et al., 2014] 
There are two major classifications for strokes: ischemic and hemorrhagic [Gomes and Wachsman, 2013]. The former is caused by the reduction in the blood supply while the second is typically caused by a disruption in one of the vessels.

According to the WHO [Organization et al., 2014] in 2012 strokes were the second leading cause of death in the world with 6.7 million casualties, with an increasing evolution compared to the analysis done in 2000 (see Figures 5.1 and 5.2).

In the time period that the Spanish Institute of Statistics (INE) has gathered information (1980-2014), the number of deaths per year grouped by age ranges can be observed in Figure 5.3. A pattern can be extracted from the analysis: global deaths caused by stroke in Spain are declining smoothly but the profile of the patients by their age is changing, as people is dying older by this cause.



Figure 5.3: Deaths by age per year in Spain from 1980 to 2014

\subsubsection{Neurological scales applied to stroke patients}

Neurologists have defined different protocols to be able to determine the severity of their patients when they are affected by a stroke. Different scales are used in order to extract relevant information in key aspects of these kind of patients: functional and neuronal activity of the patients, subaracnoidean hemorrhages, Parkinson's disease, disabilities, geriatric depression, etc.

These aspects are typically analyzed by questionnaires that are filled by doctors gathering answers to several questions, actions or test from the patients. The most typically used scales are described in the following sub-sections. 


\section{National Institute of Health Stroke Scale (NIHSS)}

This scale has been developed by the National Institute of Health (NIH) and consists of 15 questions that the doctor has to fill depending on the actions observed in the patient regarding language, coordination, muscle paralysis, etc. Each of the questions gets a score from 0 to $\mathrm{N}$ where $\mathrm{N}$ is the number of possible options, or 9 if the question is not relevant (e.g. an amputation in a test for limb movement). The maximum value for NIHSS is 42 .

A higher score on one question means a higher severity for that particular test. A global score is calculated by the sum of all the values for the questions. According to [Hage, 2011] the severity of the patient can be deducted from the values in Table 5.1.

\begin{tabular}{|c|c|}
\hline Severity & NIHSS value \\
\hline No Stroke & 0 \\
\hline Minor Stroke & $1-4$ \\
\hline Moderate Stroke & $5-15$ \\
\hline Moderate to Severe Stroke & $16-20$ \\
\hline Severe Stroke & $>21$ \\
\hline
\end{tabular}

Table 5.1: Stroke severity depending on NIHSS value

\section{Barthel scale}

The Barthel scale [Mahoney, 1965], also called Barthel Activities of Daily Living (ADL) index, is used to measure performance of an individual in ordinary tasks. The test contains a valuation from the doctor with the help of the patient of 10 questions regarding, for example, feeding, dressing, going to the bathroom, etc.

Each question is marked with 0,5 , or even 10 or 15 points, over regulated values for particular answers. Lower values indicates more dependency of the person while higher ones ensure more independence. This scale ranges from 0 to 100 and a higher value indicates that, after discharging the patient, that person will be able to do ordinary tasks by itself.

\section{Modified Rankin scale (mRs)}

As the Barthel Scale, the Modified Rankin Scale (mRs) [Van Swieten et al., 1988] centers the evaluation on the development of ordinary tasks after suffering a stroke or other neurological disabilities.

This scale ranges from 0 to 6 , where 0 means "No symptoms" and 6 means "Dead". It is also evaluated by the doctor by doing some tests to the patient. 
Chapter 5. Applying H2A: Human Health Analytics to analyze the evolution of stroke patients

\section{Canadian Neurological Scale (CNS)}

The Canadian Neurological Scale [Cote et al., 1986] was introduced as a tool to monitor patients in the acute phase of a stroke.

This scale ranges from 0 to 10 . A higher value in the scale means that the patient is healthier. It comprises values for consciousness, speech, and motor functions.

\subsection{Extracting scale values from Electronic Health Records}

$\mathrm{H} 2 \mathrm{~A}$ has been tested in this particular use case using anonymized data from real patients suffering from strokes.

\subsubsection{Implementing the neurological scales descriptor}

The neurological scales descriptor creates a pipeline of annotators to detect individual neurological and more general scales that are used in the examination of patients in the neurological unit of hospitals (see Figure 5.4).

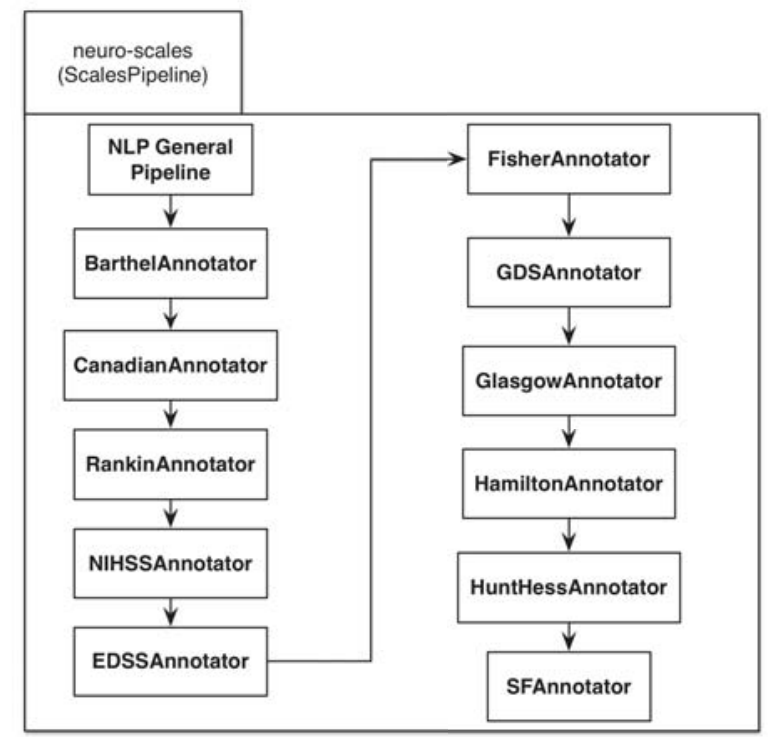

Figure 5.4: Neurological scales pipeline annotators

Apart from the annotators developed to detect the most commonly used scales for stroke patients, annotators for these other scales have been developed: Expanded Disability Status Scale (EDSS) to analyze the disability of the patient; Fisher and Hunt \& Hess scale to detect the amount of subarachnoid hemorrhage found in Computerized Tomography (CT) scans; Global Deteroration Scale (GDS) to analyze the deterioration of the patient; Glasgow Comma Scale 
(GCS) to asses the consciousness of the patient; Hamilton to analyze if the patient suffers from depression; and the SF Scale to detect the global health status of the patient.

This descriptor has been developed to be used in this particular use case and integrated in H2A's architecture.

\subsubsection{Distribution of the scales on the different document sections}

The EHRs analyzed contain discharge information for stroke patients written in free text. The information is organized in this documents in 9 different sections, which are typically used among practitioners: History, Disease, Evolution, Exploration, Neurological Exploration, Clinical Judgement, Tests, Follow-up and Treatment. Each of these sections contains information about the disease, signs or symptoms, laboratory tests and other relevant information that doctors have written.

The information on the scales can appear in different sections of the clinical narrative so the parsing method has to find it wherever it appears. Depending on the scale that is written, particular values for each single question in the form asked to the patient can be explicitly mentioned, while for other scales or patients may only figure the aggregated final value for that particular scale. This affects the parser developed as it has to work seamlessly with different types of scales. Some examples of the written information are:

- NIH: 5

- 24h postinfusión: NIHSS: 1-2-1-1-1/2-1-0-0-0/0-0-2-0-2: 15

- Al alta: NIH 2 Rankin 1 Canadiense 10. Barthel 100.

- $\mathrm{mRS}=5$ Canadiense: 3,5 NIH: 0-2-0-0-2/2-0-4-0-4/0-0-2-2-2 = 20

- Mejoría importante de la sintomatología (NIHSS 1)

- NIHS: 1-1-0-1-2-2-4-0-4-0-0-2-0-2-2

Barthel, Modified Rankin and Canadian Neurological scales all the values contained are the final aggregation of the values for the questions (ie. the dataset only contains the final value of the scale). For NIHSS, some patients only have the aggregated value, others have the value for each of the 15 questions and the value assigned by the doctor, and others contain the disaggregation of the questions without the final value for the scale.

Some of the NIHSS values contain the disaggregated values for the questions but do not contain 15 values, one for each question. These values have been excluded from the analysis due to the impossibility to objectively assign the value to a question. Although these particular measures have been excluded, they are really important outside the scope of this analysis, as can be used to reduce typing errors from doctors or to alert them in particular cases, so the information is stored accurately. This is important for the completeness of the reports and the veracity of the data, which indirectly affects the evolution and treatment given to the patient. 


\subsection{Automatic identification and analysis of patients' evolu- tion}

By using H2A with the integrated components to detect these neurological scales, it is possible to automatically detect the scales associating their corresponding values to each of them. As for NIHSS values in many cases the disaggregated values for each of the questions in the form are stated, these values are stored with their corresponding question and actual meaning of the particular values as it is expressed in the questionnaire.

The use of UMLS [Humphreys and Lindberg, 1993] to detect these types of scales is not powerful enough, although it can serve as a link to enrich the annotations and to aggregate this data with other sources of information. Therefore, it has not been used yet in the detection or to enrich the annotations mainly due to the use of different CUIs for the same concept of an scale. In the case of $\mathrm{H} 2 \mathrm{~A}$, the detection of the scales generates an independent UIMA annotation unlinked to the rest of the medical terms.

Together with the annotations and structured information of the patients an analysis on the evolution of the patients analyzing the values at admission and the values at discharge (or death) has been conducted. The analysis of the evolution of the patients (see Figure 5.5) is performed only with the automatic analysis and extraction of the neurological scales from the free-text included in the Electronic Health Records. The information has been extracted by the use of the aforementioned neurological scales descriptor which extracts the values of the different scales.

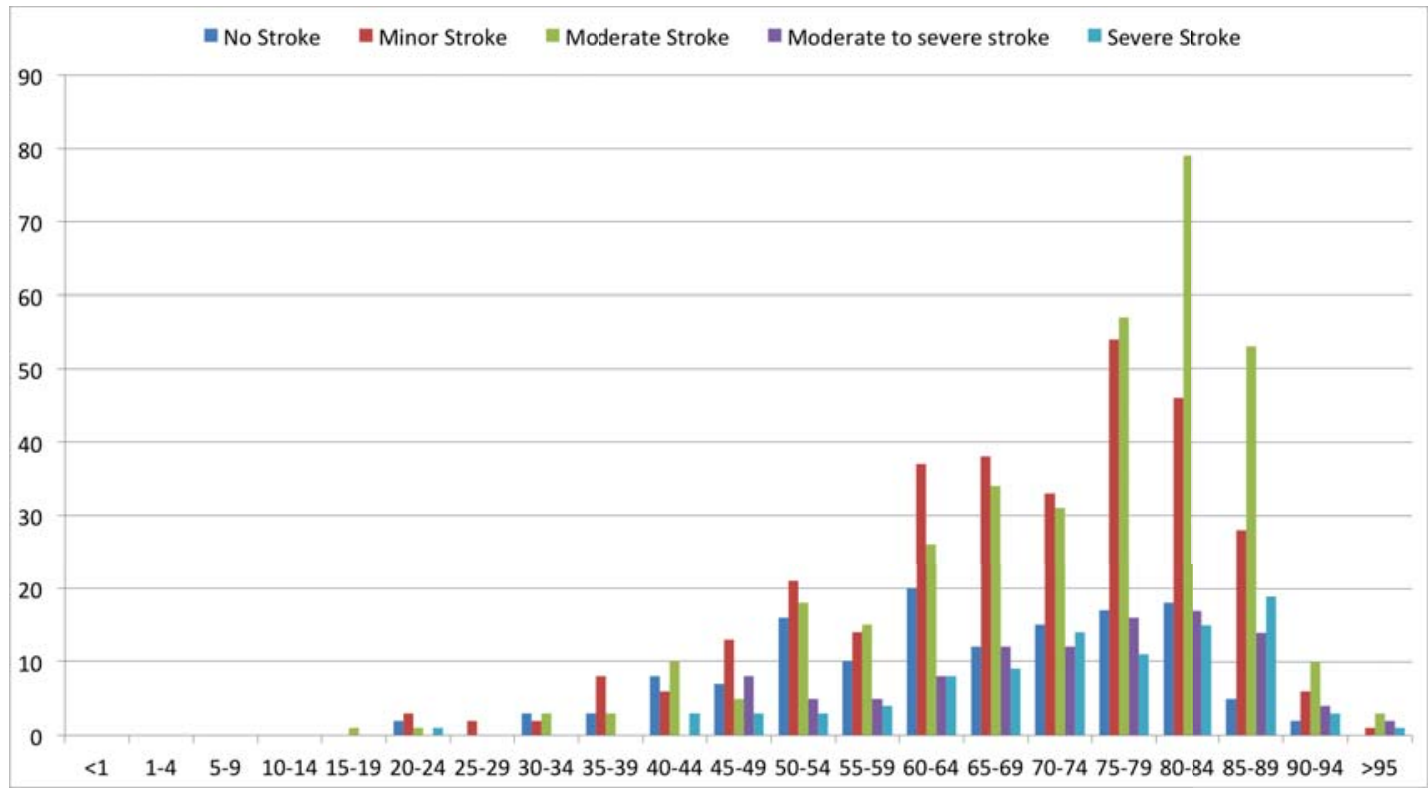

Figure 5.5: Identified severity of the patients by age 


\subsection{Implementation of an end-user application}

The development of a complex use case has to be complemented with a useful end-user application that allow end-users to interact with the information structured in the analysis process.

The definition of an end-user application allows the use of all the structured data gathered at the core of the architecture by end-users (doctors, practitioners, etc.).



Figure 5.6: General visualization of the web application

A web application has been developed as a proof-of-concept of the possibilities that $\mathrm{H} 2 \mathrm{~A}$ brings to the healthcare sector. This web based application has been developed as a full-text criteria-based search engine, in which the users can look at the patients information and perform complex searches while refining the results to obtain the desired results. The main screen of the application can be seen in Figure 5.6.

This application has been thought as a web-based operating system in which each user has a login and all its preferences are properly stored after each session. Several specific applications can be developed on top of this web service, depending on the necessities of each service: Emergencies, Consultations and Hospitalization. These services typically have different needs of managing the information.

In the general case, two applications (on the left of the image) have been developed: a search engine, shown as the main window, and a viewer application to read EHRs.

When initialized, the search engine automatically loads the information of all patients and shows a table with some basic information per patient: its id, birthdate, gender, current age and the ranking in the search performed. Options to refine the search by gender and age are also displayed. A plus button indicates the option to create new criteria which allows users to select 
Chapter 5. Applying H2A: Human Health Analytics to analyze the evolution of stroke patients 5.5. Implementation of an end-user application

what and where to find it. When clicking the plus button to add search criteria, a new window appears (see Figure 5.7).

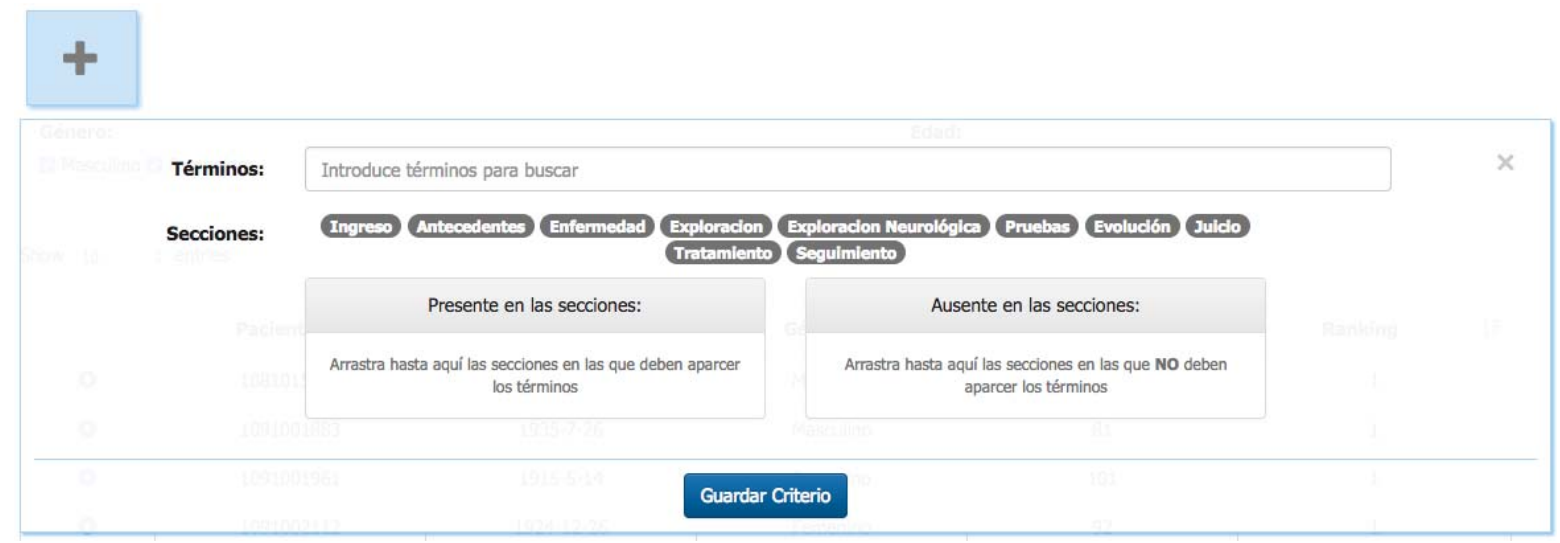

Figure 5.7: Selection of criteria search with search box and the sections of the documents

The criteria filter is simple: a text box in which users can introduce the terms to be searched, separated by commas, and a list of sections that typically appear on the EHRs and two boxes where the sections can be moved, in order to indicate if the terms have to be present or if the terms must not be present on those particular sections.



Figure 5.8: Selection of patients for a NIHSS search

Users can then introduce the terms they want and save as many criteria boxes as they want in order to filter out the results. Once a criteria is saved, a new box is created and the 
criteria can be edited, deactivated or deleted (see Figure 5.8). The results shown on the table are automatically updated to reflect what is selected on the search criteria.

Once the results are the ones desired, after applying the corresponding criteria, the users can explore individual patients by clicking on the plus button on the left of each row. A new table is then displayed with the reports indexed for that patient (see Figure 5.9). For each report, its id, the unit that generated the report, the date it was written, and the age of the patient are shown. There is also additional information with the different scales found in that particular report, including the minimum and maximum values found, and the evolution of the patient represented in a graph, with green lines representing an improvement in the patient's condition, and red lines representing the contrary.

\begin{tabular}{|c|c|c|c|c|c|c|c|c|c|c|c|}
\hline & & Pacient & ఏ! & Fecha de nacimiento & $\| 1$ & Género & It & Edad Actual & If & Ranking & IF \\
\hline \multicolumn{2}{|c|}{$\bullet$} & \multicolumn{2}{|c|}{2435} & \multicolumn{2}{|l|}{$1930-12-9$} & \multicolumn{2}{|l|}{ Masculino } & \multicolumn{2}{|l|}{87} & 1 & \\
\hline \multicolumn{2}{|c|}{$\bullet$} & \multicolumn{2}{|c|}{3256} & $1932-12-16$ & \multicolumn{3}{|c|}{ Femenino } & \multicolumn{2}{|l|}{85} & 1 & \\
\hline \multicolumn{2}{|c|}{$\bullet$} & \multicolumn{2}{|c|}{251} & $1931-6-19$ & \multicolumn{3}{|c|}{ Masculino } & \multicolumn{2}{|l|}{85} & 1 & \\
\hline \multicolumn{2}{|c|}{$\bullet$} & \multicolumn{2}{|c|}{123} & $1930-2-31$ & \multicolumn{3}{|c|}{ Masculino } & \multicolumn{2}{|l|}{87} & 1 & \\
\hline \multicolumn{2}{|c|}{$\boldsymbol{\theta}$} & \multicolumn{2}{|c|}{2154} & $1930-12-24$ & \multicolumn{3}{|c|}{ Femenino } & \multicolumn{2}{|l|}{86} & 1 & \\
\hline & Informe & Unidad & Edad & Fecha de Informe & Escala & Máximo & Minim & & & & \\
\hline \multirow{4}{*}{$\begin{array}{l}\text { 류⼆ } \\
\text { Ver }\end{array}$} & \multirow{4}{*}{1253436} & \multirow{4}{*}{ ICT } & \multirow{4}{*}{80} & \multirow{4}{*}{$2012-12-28$} & Nurn & רת & & \multicolumn{2}{|c|}{${ }^{40}$ IS chart by amCharts } & & \\
\hline & & & & & & & & & & & \\
\hline & & & & & BARTHEL & 1 & 1 & & & & \\
\hline & & & & & RANKIN & 2 & 2 & & & & \\
\hline
\end{tabular}

Figure 5.9: Expanded information for a patient showing the evolution of the patient in each scale

In this search engine, the capabilities included in the architecture make it possible not only to perform searches by the selected terms, but also to include in the results many more patients complying with the criteria by looking for synonyms, expanding acronyms, and so on. This application shows the possibilities of developing applications using the capabilities of $\mathrm{H} 2 \mathrm{~A}$ architecture.

\subsection{Discussion}

The application of the methods developed that have led to the creation of $\mathrm{H} 2 \mathrm{~A}$ architecture, which has been successfully tested in the healthcare domain. The addition of new modules developed in H2A to semantically complement the information stored in EHRs have been introduced. The application of these components has been studied together with a stroke department by analyzing the evolution of patients in last few years. Automatic extraction of information and the analysis of the stroke scales can give powerful information to the doctors, specially when aggregated to other information.

This study has shown that the extraction of these values for the patients can lead to a global study on the procedures and the performance of the treatments given to the patients and 
when mixing this information with specific procedures and treatments can bring new approaches to save more lives.

Possible future work will go into that particular direction, trying to extract all the given treatments and procedures to particular sets of patients that will enlighten more relevant information on the treatments given to a population that is being well treated, but that the incidence of the cases will increase very quickly in the following years. Future tools may be developed to aid doctors decrease typing errors when writing the different values of the scales by advising on the particular values to be stored and the evolution of the patients.

A complete end-user application developed in a web environment has been created in order to access to the information as a search engine, providing some filters on specific criteria to analyze documents in the area of stroke patients, extracting information on their evolution, as shown in the study presented. Thanks to this application, doctors, practitioners and other professionals in the sector can access relevant information of the patients. This end-user application fulfills the requirements to complete Objective $\mathrm{O} 4$.

The following publication has been one of the contributions on this research demonstrating the application of $\mathrm{H} 2 \mathrm{~A}$ in a particular use case:

- Gemma Reig Roselló, Roberto Costumero, Héctor Ambit, Consuelo Gonzalo, Ernestina Menasalvas, Santiago Trillo Senin, Jorge Gómez Zamora, Guillermo Fernández, Gustavo Zapata Wainberg, José Vivancos Mora. Aplicación de la computación cognitiva para el análisis de datos masivos (Big Data) extraídos de los informes de alta de una unidad de ictus. In LXVIII Reunión Anual Sociedad Española de Neurología, 2016. 


\section{CHAPTER $6 \square$}

\section{Commercialization: Accessing the market}

\subsection{Introduction}

Research has always been generating new knowledge to expand or interpret the world in which we live in. Last decades, as capitalism has become the standard way of countries, companies and users interact, new companies emerging from different fields have disrupted the way of working in different domains.

Digitalization over the industrial revolution has allowed for the technical fields to define a new type of newly created companies, small in their conception, very disruptive, called Startups. Many of the most well-known companies in the digital world, such as Google, Facebook or LinkedIn, were created as Startups and have made profound changes in the way businesses operate nowadays.

As an example, Google, just to mention a case, emerged from the Research performed at Stanford University. This, is one of the many examples that research, particularly in technological fields, has evolved together with solid business plans in order to create new ventures that have transferred the technology obtained in the research phase into society.

This principle of transferring research results should be the standard outcome from thesis in the academia and this vision is shared by the author. As part of the development of the thesis, not only research tasks have been performed, but also business development courses from Universidad Politécnica de Madrid and EIT Digital PhD. programs have been received.

These continuous learning on business related areas has led to the development of a complete business plan that aims to analyze the market and propose a business model in order to transfer the results obtained during the development of the thesis into the society. This business plan is focused on the Spanish healthcare market.

\subsection{Executive summary}

\section{Previous works}

Hospitals, clinics and research centers and institutes in the healthcare sector accumulate more than 150TB of data (equivalent to more than 76,000 High-Definition (HD) movies) from pa- 
tients in different formats. This data is currently not being exploited for decision-making processes that allow a global observation of diseases and treatments.

Medical practitioners generate the equivalent to 10TB of data yearly [Winter et al., 2010] and it is based on the evidence of diagnostic tests performed on a patient to diagnose, together with the previous experience from a professional that emits its clinical evaluation. Most of the data generated is not used in global decision-making processes over a target population or individuals, giving diagnoses over previous observations realized over the entire population. The use of DSSs will allow better assistance quality thanks to the reduction of recurrent consultations, due to higher accuracy on diagnoses and treatments given by medical staff.

Our experience as a research group and the different collaborations with two renowned public hospitals in the region of Madrid, have allowed us to develop, improve and detect real world problems in the healthcare system. This has led to an evolution of the project to adapt the commercialization and solution to the market needs.

\section{Business opportunity}

The amount of data generated in the healthcare sector means that doctors would need 21.7 hours per day in order to obtain all the knowledge generated in their field to improve clinical assistance [Post, 2015]. This is an underuse of one of the most important resources in this sector: data from daily activities.

H2A: Human Health Analytics proposes a technological solution that allows the extraction of knowledge which is hidden in Electronic Health Records and to offer it, in a quick and simple way, to healthcare professionals. Moreover, H2A offers a natural language search engine that allows the recovery of EHRs and reports from a given query. An statistical analysis of the retrieved information is also presented, taking into account the history of the patient and other relevant information, differentiating our solution from the rest of competitors.

\section{Market analysis}

The main market for H2A is the Spanish healthcare system, which in 2013 had an assigned budget of 93,048 $\mathrm{M} €$ (both private and public investments) to the assistance of protected population. Particularly, finance will focus on capturing part of the investment assigned to IT, which represented a grand total of 660,972,000 € in 2014, incrementing in 6.57\% from 2013 budgets. In this scenario, pressure made to the healthcare system due to ageing population increases the necessity of finding solutions that allow a reduction of costs by a rational use of the resources (reducing the number of consultations and explorations).

The implantation of Electronic Health Records has changed the view on the modern Spanish healthcare system, which aims for improving consultation processes. Analysis of the data from Spanish hospitals needs from the development of a new technology that is able to analyze automatically big volumes of data and, is here, that according to some studies the future use of 
Big Data technologies will give more value to the daily clinical practices.

\section{Business model}

Monetization of the product will be done thanks to: I) a fixed cost upon contract signature; and II) a fixed monthly cost per maintenance, support and updates. More precisely, customers will be offered different plans based on the number of EHRs they can include into the system within a year. This figures can be augmented if needed by covering the extra processing and storage costs due to the increase in the data uploaded.

\section{Marketing}

Due to the extensive knowledge acquired thanks to the proof of concept developed in collaboration with different hospitals, we can offer a competitive tool in the healthcare market.

H2A offers capabilities to understand and interpret the text written in the EHRs and potential customers can benefit from this product. Different types of plans in order to adapt to the necessities of the sizes of the hospitals and their budgets have been developed.

\section{Financial aspects}

A complete financial analysis of the company has been made. The prevision of the hirings, expenses and selling plans, give a clear vision on what are the financial necessities of the company. From the third year, the stability of the company increases with a steady growth. This analysis is complemented with a summary of earnings and loses.

In order to guarantee proper daily activities in the company and the stabilization of the treasury, an initial investment of $200,000 €$ is needed ( $100 \mathrm{~K} €$ yearly). A similar investment later on, would guarantee the stability of the company in its first 5 years, considering a breakeven during the 6th year.

\subsection{Strategic plan}

\section{PEST Analysis}

Healthcare systems around the world are targeting new challenges related with the increase in healthcare expenses derived from factors like the increasing life expectancy by: I) An aging population; II) Chronic diseases; and III) Scientific and technological innovation.

There is a historical increasing trend in healthcare expense in Spain: In 2000 it represented $7.21 \%$ of GDP, whereas in the following ten years a sustainable growth has increased 
that figure up to $9.66 \%$ in 2010 . Nevertheless, from that date on there is a progressive decrease in healthcare expenses relative to the GDP, from $9.66 \%$ in 2010 to $8.90 \%$ in 2013, due to budget restrictions in the healthcare system due to the economic crisis.

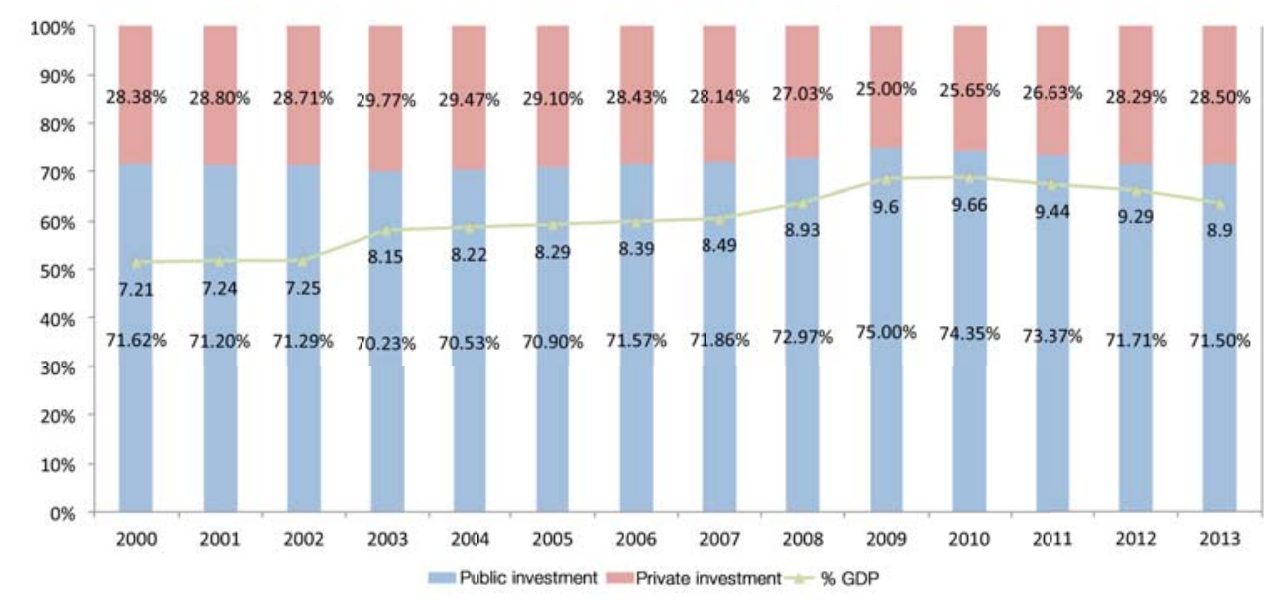

Figure 6.1: Total healthcare expenses in relation with GDP in Spain, 2000-2013. Source: Prepared by the author from OCDE, Health Data 2015, and MSSSI, Sistema de Cuentas 2013.

Most of the expenses $(71.50 \%-66,521 \mathrm{M} €$ ) in the healthcare sector is from public investment (see Figure 6.1). Private expenses are just 28.50\% - 26,527 $\mathrm{M} €$ from the total. In terms of their evolution, private investment has been growing in the last decade, from $2.05 \%$ GDP in 2000 to $2.50 \%$ in 2013 . This growth has significantly covered the decrease in public investment.

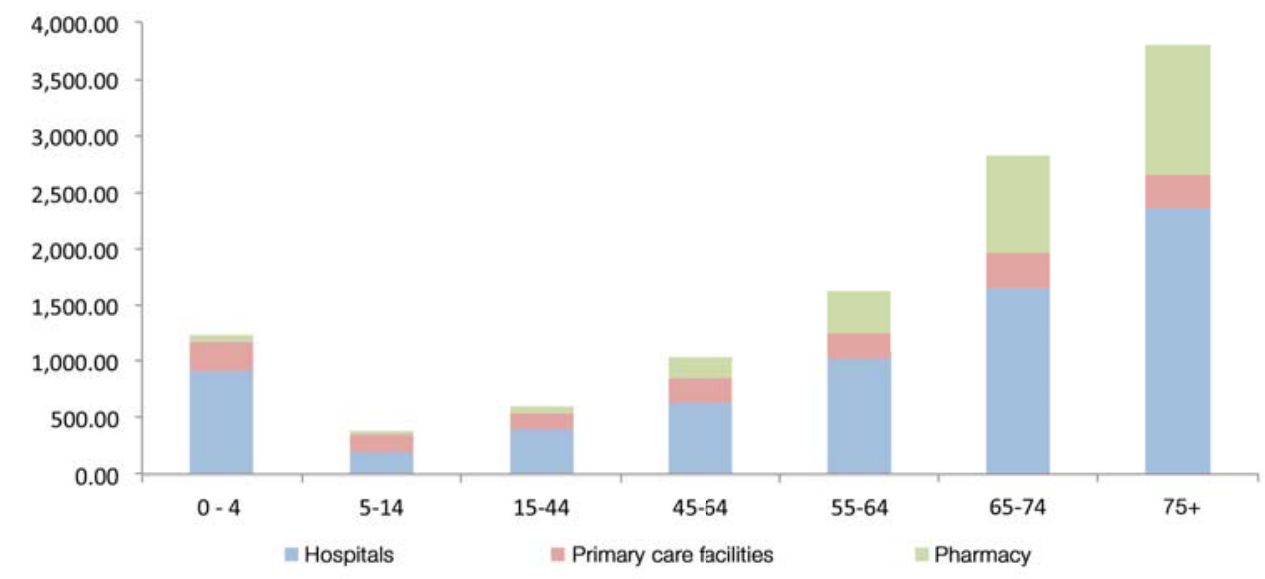

Figure 6.2: Healthcare expenses (in euros) by age in 2010. Source: Prepared by the author from Fundación BBVA 2010.

According to [de Cos and Moral-Benito, 2011], one of the factors in the evolution of the expenses in the healthcare sector, is demography. These expenses increase with the age (see Figure 6.2), so as the population is ageing, this change will affect the global expense in healthcare. Moreover, an increase of elderly people (see Figure 6.3) will increase the pressure on the healthcare system, even if the expenses per person are the same for any given age group. 


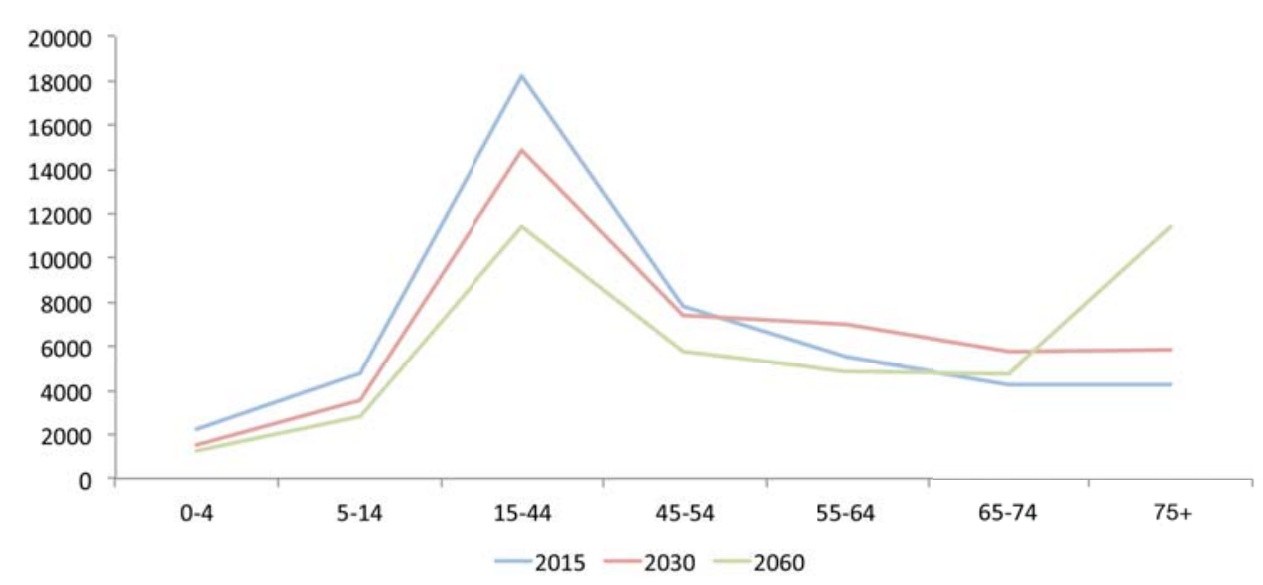

Figure 6.3: Estimation on Spain's population by age group in 2015, 2030 y 2060. Source: Prepared by the author from data from INE, 2015.

Global budget for IT taking into account all the regions has increased 5.82\% (660,972,000 $€)$ in 2014 compared to the one in 2013, according to Índice SEIS 2014 [Codina et al., 2014]. From this budget, the corresponding expense in IT per person was $14.31 €$ in 2014 , increasing $5.75 \%$ from 2013. With these data in hand, it is clear the effort made to integrate IT in the healthcare system, as part of its modernization plan.

EHRs implantation brings a more agile and secure management that its paper-based predecessor. EHRs management in Spain is dependent on the different regions and there is no current interoperability among the different implemented systems, even if in most cases the EHR systems implemented are standard solutions (eg. SELENE [Siemens, 2015] or customized developments done per hospital [MSSSI Ministerio de Sanidad et al., 2010]).

Nowadays, Big Data Applications offer a huge potential of improving efficiency and assistance quality in the healthcare sector [Kayyali et al., 2013]. Nevertheless, analyzing data in Spanish (not exclusively) hospitals needs the development of several technical requirements like: I) Natural Language Processing for Spanish applied to health related documents; II) Imaging analysis; and III) Integration of the information extracted in texts and images. Furthermore, analysis of data has to comply in Spain with the corresponding regulations in the management of personal data (art. 12 LOPD, art. 20 - 22 RLOPD), regarding anonymization, access, and storage of patients' data.

$\mathrm{H} 2 \mathrm{~A}$ analyzes unstructured data to allow professionals to make evidence-based decisions. The software creates a homogeneous solution to search patients' data by interpreting natural language found in EHR, reports, laboratory tests.

\section{Microenvironment analysis: Potential customers}

According to Ministerio de Sanidad, Servicios Sociales e Igualdad [MSSSI Ministerio de Sanidad, 2015], there are 81 active hospitals only in the region of Madrid. There are also 19 institutes 
and biomedical research centers in the same region.

Density of healthcare professionals per 1,000 inhabitants is established in 0.8 primary care doctors and 1.7 specialized doctors. In nursing, values remain at 0.7 in primary care and 2.8 in specialized care. In the last years there is an obvious decrease of professionals, which is more pronounced among the nurses [MSSSI Ministerio de Sanidad, 2013].

Primary care units attended 375 million consultations just only in 2013 and only 4 out of 10 people got their consultation on the same day, but among those who did not, the average waiting time was 3.5 days. With these ratios and the increasing volume of data generated at hospitals, healthcare professionals do not have enough time to update their knowledge with new advances, increasing the time and costs in patients' assistance.

Potential customers can be divided in different groups, according to their topology or their investment type. According to their topology:

- Hospitals and primary care centers.

- Institutes and research centers in the area of biomedical activities.

According to their investment type:

- Public hospitals.

- Private hospitals.

Furthermore, there is a classification based on the quantity of data (Electronic Health Records and laboratory tests) generated on a yearly basis:

- Small hospital: 100,000 reports and 1,000,000 laboratory tests.

- Medium hospital: 250,000 reports and 4,000,000 laboratory tests.

- Large hospital: 500,000 reports and 7,500,000 laboratory tests.

- Extra-Large hospital: 1,000,000 reports and 12,000,000 laboratory tests.

\section{Microenvironment analysis: Providers}

As the product is a software which is developed for hospitals, the only need is for the technicians in the hospital and their hardware/software providers to give $\mathrm{H} 2 \mathrm{~A}$ access to the servers and their databases. 


\section{Microenvironment analysis: Competitors}

Among possible competitors the most relevant categorized by their potential threat are introduced: with similar products, with potentially similar products, technology enablers, and substitutes.

\section{Competitors with similar products}

In this category there are competitors whose product is very similar to H2A. These are direct competitors and, in some cases, they are already in the market.

H2A's main competitor is Savana Médica [Savanamed, 2016] [Medrano et al., 2017], a Spanish startup constituted on September, 12th 2014, which currently employs 8 people and offers solutions to reuse clinical data.

Savana Médica offers a first platform named "Savana Consulta", which supports evidencemade decisions for doctors to use in real time. The product consists in a search engine developed to and with doctors. This first product is a search engine that searches for relevant information for the data introduced by physicians, in which they only analyze information generated in EHRs written in natural language. They have developed a commercial product that is being implemented in the well-known Hospitales de Madrid network.

They are actively developing two more applications: I) "Savana Research", targeting research data; and II) "Savana Manager", targeting hospital managers.

\section{Competitors with potentially similar products}

These competitors have deployed products in the areas of Natural Language Processing not focused in the healthcare area and could expand in the sector. One of this kind of competitors is Bitext [Bitext, 2016], which is a company working on the NLP field performing Sentiment Analysis. It was constituted 8 years ago and currently employs 9 people.

\section{Technology enablers}

These are companies that have developed technology to be used by third-parties in order to develop products that compete with $\mathrm{H} 2 \mathrm{~A}$.

IBM has developed Watson [IBM, 2011], [IBM, 2014], a cognitive system that could be used as an expert system to feed a search engine. Although that they are currently developing the health support for Watson, the implementation is being done mainly in English in the American market. Of course, the economical and personal capabilities of IBM suppose a future threat are completely different to the ones offered at the university. 


\section{Substitutes}

Selene Clinic [Siemens, 2015] is a product developed by Siemens which is currently implemented in most hospitals to acquire and recover EHRs. This system has forms to be filled out and contains a basic search engine to recover EHRs by patients' identifiers or personal information, but not by relevant medical aspects.

Products like Selene Clinic can certainly evolve to offer more complete services allowing professionals to search by the symptoms or treatments.

$3 \mathrm{M}$ offers also a codification system for diseases to the hospitals. This is another of the possible substitutes in the healthcare sector, although this technology is currently only available in English.

\section{Microenvironment analysis: Entry barriers}

Entry barriers to get to the market are:

1. LOPD regulation which is particularly strict in this sector due to the nature of the data used.

2. Lack of standard formats in the data sources that could affect the implementation of the system, although the tendency shows that there are an increasing number of customers applying certain standards.

3. Public administration dependent customers have a restriction on the signature of contracts without public competition which is of $12,000 €$. Late payments suppose another risk factor.

4. Hierarchy in management may provoke that what is important for certain people, is not for others: end-users could demand a solution but the global managers would hesitate or vice-versa.

5. Experience in data management that guarantees results based on the necessity in the sector to work with information which is $100 \%$ trustable.

\section{SWOT analysis}

As a conclusion on the analysis shown in Table 6.1, the following strategies are extracted:

- Create a product for the market that is able to reuse data generated at a competitive price.

- Create an advisory board including professionals in healthcare sector, like doctors and IT experts. 


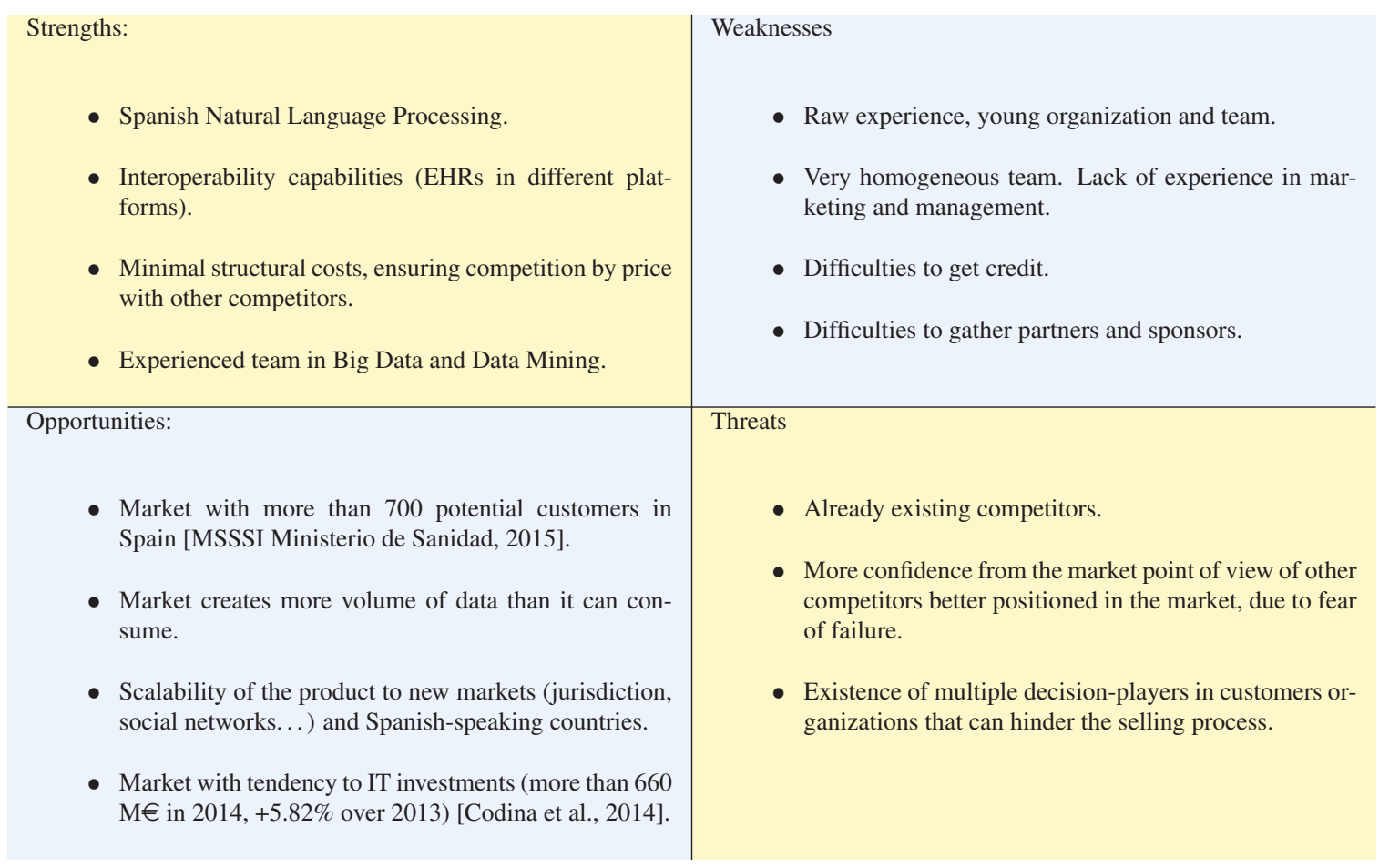

Table 6.1: SWOT Analysis

- Create an heterogeneous and multidisciplinary team by hiring non-technological profiles in the first years.

- Elaborate an strategic and a marketing plan to access to all the decision-makers and gain focus against already existing customers.

\section{Conclusions: Competitive advantages}

The following competitive advantages can be extracted from the information already shown:

Big Data experience The team already has proprietary algorithms and innovative methods in NLP, with more than 20 years of experience in both areas.

Contacts among potential customers The team has already established many contacts with different potential customers who are interested in the proposed solution.

Patenting A process of analysis, identification and development of Intellectual Property Rights (IPR) patenting of the methods developed.

Human Centered Design Final users are included in the design of a web application and they are part of the team in the development of the product. 


\subsection{Marketing}

\section{Strategic plan}

Existing regulations and the resistance from the potential customers to information leakage, have led to an on-site install approach. This limits the decisions made at a hospital level, but the adaptation to customers needs is key.

A customizable product that is able to adapt to particular needs from each customer can be offered with a fixed cost upon installation and a fixed monthly cost per maintenance.

\section{Products}

H2A has a product under development, an intelligent search engine that allows healthcare professionals to recover Electronic Health Records and reports that correspond with the realized query. Proposed solution is able to:

- recover the required information in an effective way,

- order the results based on their relevance,

- show relationships among different sources, and

- show basic statistics.

First phase in the development is focused on the search of information from oncology, neurology and radiology. Currently developed use case, will allow neurologists to explore the cases where patients suffered from a stroke along with several basic statistics relevant to these kind of patients.

\section{Prices}

Prices are based on two aspects: I) size of the service using the product, and II) functionalities acquired.

Customers can access a product in which the system will be able to process and return EHRs and reports written in natural language. It will also be able to analyze laboratory tests written in the EHRs

Taking these criteria into account, the pricing policy is the following:

- A fixed cost upon installation of the product.

- A fixed monthly cost per maintenance, support, and updates. 
Table 6.2 shows the pricing plans.

\begin{tabular}{|l|c|c|c|c|}
\multicolumn{1}{l|}{} & Plan S & Plan M & Plan L & Plan XL \\
\hline Maximum number of reports & 100.000 & 250.000 & 500.000 & 1.000 .000 \\
\hline Maximum number of laboratory tests & 1.000 .000 & 4.000 .000 & 7.500 .000 & 12.000 .000 \\
\hline Price over signature & $45.000 €$ & $62.000 €$ & $108.000 €$ & $155.000 €$ \\
\hline Fixed maintainance price per month & $2.500 €$ & $4.000 €$ & $6.000 €$ & $7.500 €$ \\
\hline Final price yearly & $75.000 €$ & $110.000 €$ & $180.000 €$ & $245.000 €$ \\
\hline
\end{tabular}

Table 6.2: Pricing plan: only reports version (annual prices without VAT)

Prices are established following these indicators:

- Processing cost of a report,

- Storage cost of a report,

- Fixed costs on the development structure,

- Other indirect costs of developing the product, and

- Contribution margin.

As an example, a typical hospital falling into Plan $L$ would spend $180,000 €$, which is a $0.001 \%$ of a medium size hospital's budget.

\section{Distribution}

The installation has to be done on the customer premises, although there always exists the possibility to offer a cloud-based solution, depending on the customer needs and preferences, and if the regulation updates.

Either way, anonymity of the data collected from the patients in the different centers is ensured, without losing the quality and effectiveness of the models created using that data.

\section{Selling plan}

Table 6.3 shows a prediction of customers signing up for the different packages.

\begin{tabular}{|l|c|c|c|c|c|}
\multicolumn{1}{l|}{} & Year 1 & Year 2 & Year 3 & Year 4 & Year 5 \\
\hline Plan S & $\ldots \ldots$ & & 1 & $\ldots$ & $\ldots$ \\
Plan M & 1 & 1 & 1 & & 1 \\
Plan L & 1 & $\ldots$ & 1 & 2 & 1 \\
\hline Plan XL & & & 1 & & \\
\hline Total Reports & 1 & 1 & 3 & 2 & 1 \\
\hline
\end{tabular}

Table 6.3: Selling plan - New customers 
Customers would be approached through doctors and chiefs of services to perform proofs of concept. This way, doctors can test the technology and can adapt the solution to their needs. Once they are convinced, 2 - 4 estimated meetings with hospital managers are needed to evaluate global operation of the solution at a hospital level. This way, more areas are covered with a lower cost, improving the obtained results due to the use of more information. These meetings can take up to 8 months.

Payments to providers are done on a 60-day period, while incomes can take as long as 2 years if it is a public managed customer. In this scenario, in order to limit effect on treasury, factoring will be performed with a bank credit with a limit of $250,000 €$ growable to $500,000 €$ from the second year, obtaining more cash with an estimated $5 \%$ loss.

\subsection{Operational organization}

\section{Critical operations}

There are several items to finish before launching operations, but the following three will determine the product success:

Financing The need for financing is key for the development of the product, specially due to the aforementioned problems in treasury if customers are public entities.

Product development To finish and package the final product as a Minimum Viable Product (MVP) with the proofs of concept explained in 6.6.

First customer acquisition To gain a first customer, preferably one of our collaborators, to get a first source of income.

\section{The team}

The team behind this research is the following:

Roberto Costumero is the responsible for the execution of the project. $\mathrm{PhD}$. Student in the areas of Natural Language Processing and Machine Learning applied to Electronic Health Records. His technical education and enthusiasm is key to develop H2A. Roberto was awarded in ActúaUPM's 2007 edition as one of the best 8 business ideas. He has also taken part in Spring's 2011 edition of Tetuan Valley Startup School. Leadership and an entrepreneurial mindset ensure the proper development of the business.

Consuelo Gonzalo is an associate professor at UPM with experience in leading research and development projects in digital image analysis, acquiring the needed know-how to develop new projects in the area, as well as a very extensive network of contacts which are 
necessary in H2A. As an added value, Consuelo has an important network of contacts in Chile, relevant for the international expansion of H2A. She is also responsible of the work packages in Big Data dissemination.

Ernestina Menasalvas is an associate professor at UPM and has dedicated her professional life to knowledge discovery in databases in different domains, focusing on healthcare in the last years. She has also managed and lead innovative projects. She has also participated and continues on international consortiums for the development of Big Data. Therefore, she is the responsible of impulse $\mathrm{H} 2 \mathrm{~A}$ and to search for financing for its development.

\subsection{Goals}

These are the goals already achieved demonstrating the potential and versatility of the product

FIS project Started the collaboration in the SOPHOS project for the analysis of unstructured clinical data. A collaboration has been performed in a FIS project entitled: "Estudio de re-análisis de imágenes y correlación entre los cambios metabólicos objetivados por Positron Emission Tomography (PET)/CT y las mutaciones conocidas en el cáncer de pulmón no célula pequeña (CPNCP)". This project was financed by research grants for healthcare projects by Instituto de Salud Carlos III and has demonstrated a need in the market and the interest of potential customers, as they are demanding these solutions.

Proof of concept in stroke unit An analysis of EHRs in stroke patients has been performed.

Award to one of the best ideas: XII ActúaUPM edition A project presented under this research was awarded with $1,000 €$ in the first round as one of the 10 best business ideas. The complete business plan developed for that purpose was among the finalists.

Adapting the technology in other fields In collaboration with Universidad de Concepción of Chile a project was developed in the environment of applying NLP to judgement texts to demonstrate the versatility of the technology. This project consisted on a web platform to search, visualize and geographically locate the content extracted from judgments.

\subsection{Discussion}

Universities are developing processes to ensure that research performed ends up in the market, transferring the technology to spin-offs or startups created as part of the technology transfer process.

The developed business plan introduced in this chapter shows the status of the market in this particular sector. The developed methods can be transferred directly by the university in close collaboration with hospitals, as the ones that have led to this research, or to a newly 
Chapter 6. Commercialization: Accessing the market 6.7. Discussion

created venture. This business plans complements the research performed as a natural evolution on the process of this thesis.

A more complete and ambitious business plan was performed as part of the participation of the research group in ActúaUPM, but a more concise and targeted to the research performed in this thesis is the one introduced here. This business plan fulfills the requirements to complete Objective O5. 


\section{CHAPTER \\ 7}

\section{Conclusions and future work}

\subsection{Conclusions}

This thesis has introduced new methods to apply NLP techniques to the analysis of EHRs. In this process and during the development of this thesis, $\mathrm{H} 2 \mathrm{~A}$ architecture has been designed and developed to overcome the different problems proposed. The research performed has led to the following contributions:

- Computational models have been generated using generic and domain specific corpora and tested in the healthcare domain. These models have been trained with OpenNLP software. A comparative study on their performance over a blind test of EHRs has been performed and the conclusions extracted show that:

- Generic corpora applied to a blind test set of medical documents clearly outperforms domain specific corpora when the former is much larger.

- Generic corpora is able to detect better sentences even when the size of the corpus is equal to the domain specific one.

- Domain specific corpora trained models outperform generic corpora on the tasks of PoS tagging and Chunker tagging when using EAGLES and Universal PoS tagsets with equally-sized corpora.

- Domain specific corpora trained models outperform the ones generated with generic corpora on the tasks of PoS tagging and Chunker tagging with EAGLES and Universal PoS tagsets, being the generic corpora much larger than the domain specific one. However, the differences in PoS tagging, show that a generic corpora can be used to train models and applied in the healthcare domain.

- Solutions for very specific problems in the analysis of EHRs have been developed. More specifically, solutions have been proposed to the problems of the detection of negated entities, the recognition of UMLS concepts and drug names in the free-text of EHRs, and the disambiguation of acronyms contained in these types of texts. New algorithms and methods have been presented in order to solve these problems.

- A new architecture to structure and extract meaningful information from the free-text contained in Spanish EHRs has been designed. The different components have been developed and joined in a common framework. This architecture has been designed to 
provide a generic environment in the structure of unstructured texts from different domains, although its main use case is in the healthcare domain. This architecture can be used in different languages by only performing small changes.

- A particular use case and an end-user application to assess the evolution of stroke patients has been implemented and presented. This application allows doctors to search for patients given different criteria in order to extract relevant information from the EHRs. This use case is a great example on the use of the technology developed, demonstrating the applicability of all the methods described in this thesis.

- Among all the different contributions, the creation of a complete business plan helps to understand the market conditions to exploit these results obtained and reuse the technology not only for research, but also to generate traction in the market.

Consequently, all these main contributions have led to the successful achievement of all the objectives planned for this thesis.

The hypothesis presented have been verified with the creation of different experiments and through the implementation of new methods.

- Natural Language Processing techniques can successfully be used to extract relevant information from unstructured texts, like those written in Spanish Electronic Health Records, without human intervention.

- Models created using generic amounts of data can be used in a very specific domain.

- Extraction of particular entities tied to a domain like healthcare can improve the detection of hidden knowledge in the EHRs.

The creation of a complete architecture that gathers solutions to the most common problems in analyzing Spanish written EHRs proposes an improvement in the existing approaches studied and extends its possible use with minor tweaks in other domains.

\subsection{Future lines}

As it has been stated, all the objectives planned for this thesis have been successfully achieved. However, even though the planned objectives are completed, the development of this work has opened new research questions that are left as future research as continuation of this work.

The identified future research works that could be done on the basis of this thesis are:

- The creation of new approaches to detect negation, hypothesis and affirmative events. The proposed solution is already outperformed by other recent studies but there is still room to improve the results obtained. The creation of new approaches for Spanish written texts, could lead to an improvement in the analysis of EHRs. 
- The inclusion of more ontologies and dictionaries to improve Named Entity Recognition. The study performed in this thesis focuses in the use of UMLS and databases obtained from [de Medicamentos y Productos Sanitarios, 2013] and SEDOM, but there are still other resources that can be used to improve the entity recognition. As a first approach, and being one of the most widely used ontologies in Spain, ICD-10 should be the first option to be evaluated.

- The improvement of the NLP pipeline generating multiple versions supporting multilingualism. This is an important step towards the integration of $\mathrm{H} 2 \mathrm{~A}$ in different countries by solving the problem of understanding different languages.

- The creation of new methods to include image analysis and structure to broaden the kind of results obtained. The proposed architecture has been done to support different kinds of unstructured data contained in the EHRs but the focus on this thesis has been on the NLP approaches to structure the text contained in the records. However, improvement of the architecture with new components able to structure and extract information from medical images is a relevant step towards a complete EHR architecture that is able to analyze all types of different unstructured data generated.

- The application in other domains could serve as a greater test of the technology and improve the implementation by generating new components and assessing the feasibility of its application to different domains. 


\section{Appendices}




\section{APPENDIX A}

\section{IBM Watson Internship Report}




\title{
Internship report
}

\author{
Roberto Costumero Moreno
}

DNI: 70816027-Q

Home Institution: Escuela Técnica Superior de Ingenieros Informáticos, Universidad Politécnica de Madrid

Host Institution: IBM Ireland 


\section{Introduction and goals}

As part of my $\mathrm{PhD}$. Studies, an internship was mandatory in order to obtain the international certification at the end of the studies. The good relationship between the MIDAS research group, where I work, and IBM Spain, brought the possibility of working at IBM Watson Core Development team based in IBM Technology Campus in Dublin, Ireland.

My research is focused on Natural Language Processing and Big Data Analytics applied to Spanish Healthcare systems, analysing clinical reports in Spanish language and developing new techniques for processing different kinds of texts written in the aforementioned language. Being IBM Watson [1] the most famous and advance technology in the field of Natural Language Processing ans Q\&A and due to the effort of Dublin's team on moving the platform towards a pure multilingual environment, it made sense to do the internship at IBM.

The main goals of the internship were:

- Support the team in the improvement of Part-of-Speech tagging in Spanish language and other generic techniques applied to other languages.

- Help to improve Dependency Parsing in Spanish providing solutions and proper parses for given questions and answers.

- Analyse the overall performance of the system in Spanish and helping to improve it by developing or proposing new approaches to the problems found.

\section{Main developments}

During the internship different types of work have been performed, including bug reporting and fixing, natural language processing analysis, performance analysis and machine learning improvements.

In the first two weeks of the internship, I went through a training at the Watson University courses, familiarizing myself with the project and the methodologies used in IBM Watson. I had also to set up the machine I was provided and the whole development environment in order to perform the tasks that I had to be performing.

During the next weeks I performed some research and analysis on using the AnCora Corpus [2] to train models with different features using the Universal Dependencies tagset [3] and obtaining different models and performances, ranging the $90-94 \%$ of accuracy for Part-of-Speech tagging. These preliminary tests were performed using a subset of $90 \%$ of the data included in the corpus as the training set, and $10 \%$ for validation. Cross-validation could have been used but as we only wanted to get preliminary results, this test was enough. An automatic tool to convert EAGLES [4] tagset to Universal Dependencies tagset was developed.

Then I was working on fixing the main scorers that were developed in an English-driven form in order to get them working in a multilingual environment, regardless the specifics of any language in particular. These fixes include changes in the code, running the tests and performing analysis on the results to check if the changes were properly made.

In these tests I found several problems related to the training and test sets related to Spanish specific constructions that may be causing problems in the experiments. These problems were fixed by removing duplicate questions and ensuring the uniqueness in the platform.

I also reviewed parse data from one of the client's input in Spanish. I performed this task because as a native speaker I am able to interpret and find better solutions to Spanish specific contents that may 
not be trivial for other members of the team, ensuring a quicker solution.

Some fixes were also done to make the system behave in a more deterministic way, so the results between two experiment runs with no changes in the code, behave the same giving the same output results. This is an important factor in the development of the platform to be able to measure the key changes and how they affect the system.

From the Machine Learning perspective, we did an analysis in the number of the features being used in the models to train the system and gathered information on the most important ones to be left into the models so the system performs better. Given this changes the system performed much better.

As we evolved in the analysis of the improvements in the platform, we end up needing to develop new tools to control the different types of questions that are input to the system. A software tool was develop to get the user's input to gather different percentages of the questions that will be used to train the system upon. As part of this process, some new variants for different questions were manually created to test the system for different purposes.

After applying the different fixes and new solutions we have been able to get the system working better in a multilingual environment focusing mainly on English, Spanish, Japanese and Brazilian Portuguese. The achievements have been succesfully reached in this period and further developments will be done by the team after the end of my internship.

\section{Conclusions}

The development of this internship has been done in an incredible environment at IBM Technology Campus working together with an amazing team. For the first time I have been able to work in a company environment dealing with big projects affecting external customers from all over the world. This internship has helped me to further understand the problems in the fields of Natural Language Processing and Machine learning. I had also the opportunity to attend a workshop with one of the customers and the experience was very fulfilling and will sure help me develop the skills to make a better job at my $\mathrm{PhD}$. Also the internationalization of the team has brought me different perspectives and it has been an incredible experience.

Future possible collaborations that could get publications will be considered as some of the work developed has not been able to be published for now. Also the experience has been very good because of the knowledge acquired of IBM Watson technology that will help me to teach a better Cognitive Systems course next year using this technology that will be offered as part of the Master studies at ETSIINF - UPM during the first semester on the following academic year.

\section{Bibliography}

[1] http://www.ibm.com/smarterplanet/us/en/ibmwatson/

[2] TAULÉ, Mariona; MARTÍ, Maria Antònia; RECASENS, Marta. AnCora: Multilevel Annotated Corpora for Catalan and Spanish. En LREC. 2008.

[3] http://universaldependencies.github.io/docs/

[4] http://nlp.lsi.upc.edu/freeling/doc/tagsets/tagset-es.html

\section{Acknowledgements}

Thanks to Consejo Social of Universidad Politécnica de Madrid for the grant given to help me perform this internship that has been of great benefit for my studies and for the University itself. 


\section{Supervisor agreement}

I, Anthony Kelly, Software Development Manager - Watson Core Tecnology, and supervisor of Roberto Costumero Moreno during the internship, hereby certify that the information supplied is accurate with the work the student has done and agree with the contents in this report.



Signature and Stamp 


\section{Bibliography}

[Accenture, 2010a] Accenture (2010a). Overview of international emr/ehr markets. http://Www.accenture.com/SiteCollectionDocuments/PDF/ Accenture_EMR_Markets_Whitepaper_vfinal.pdf.

[Accenture, 2010b] Accenture (2010b). Overview of international emr/ehr markets. https://www.accenture.com/_acnmedia/PDF-4/ Accenture-Getting-EMR-Back-East-Lane.pdf.

[Agirre et al., 2009] Agirre, E., De Lacalle, O. L., Soroa, A., and Fakultatea, I. (2009). Knowledge-based wsd and specific domains: Performing better than generic supervised wsd. In IJCAI, pages 1501-1506. Citeseer.

[Aparicio et al., 2008] Aparicio, J., Taulé, M., and Martí, M. A. (2008). Ancora-verb: A lexical resource for the semantic annotation of corpora. In $L R E C$.

[Apple, 2013] Apple (2013). Siri. http://www.apple.com/ios/siri/.

[Aramaki et al., 2010] Aramaki, E., Miura, Y., Tonoike, M., Ohkuma, T., Masuichi, H., Waki, K., and Ohe, K. (2010). Extraction of adverse drug effects from clinical records. Stud Health Technol Inform, 160(Pt 1):739-43.

[Aronson, 2001] Aronson, A. R. (2001). Effective mapping of biomedical text to the umls metathesaurus: the metamap program. In Proceedings of the AMIA Symposium, page 17. American Medical Informatics Association.

[Aronson, 2006] Aronson, A. R. (2006). Metamap: Mapping text to the umls metathesaurus. Bethesda, MD: NLM, NIH, DHHS, pages 1-26.

[Banon, 2011] Banon, S. (2011). Elasticsearch: An open source, distributed, restful search engine. URL: http://www.elasticsearch.org, (Last checked: 25-01-2017).

[Ben-Assuli, 2015] Ben-Assuli, O. (2015). Electronic health records, adoption, quality of care, legal and privacy issues and their implementation in emergency departments. Health Policy, 119(3):287-297.

[Bertram et al., 2007] Bertram, L., McQueen, M. B., Mullin, K., Blacker, D., and Tanzi, R. E. (2007). Systematic meta-analyses of alzheimer disease genetic association studies: the alzgene database. Nature genetics, 39(1):17-23.

[Bhatia et al., 2009] Bhatia, N., Shah, N. H., Rubin, D., Chiang, A., and Musen, M. (2009). Comparing concept recognizers for ontology-based indexing: Mgrep vs. metamap. Paper accepted to the AMIA Summit on Translational Bioinformatics, San Francisco. 
[Bitext, 2016] Bitext (2016). Bitext. https : / /www. bitext.com/. Last checked: 30-092016.

[Björne et al., 2013] Björne, J., Kaewphan, S., and Salakoski, T. (2013). Uturku: drug named entity recognition and drug-drug interaction extraction using svm classification and domain knowledge. In Second Joint Conference on Lexical and Computational Semantics (* SEM), volume 2, pages 651-659.

[Bollacker et al., 2008] Bollacker, K., Evans, C., Paritosh, P., Sturge, T., and Taylor, J. (2008). Freebase: a collaboratively created graph database for structuring human knowledge. In Proceedings of the 2008 ACM SIGMOD international conference on Management of data, pages 1247-1250. AcM.

[Boudin et al., 2008] Boudin, F., Torres-Moreno, J., and El-Bèze, M. (2008). Mixing statistical and symbolic approaches for chemical names recognition. Computational Linguistics and Intelligent Text Processing, pages 334-343.

[Buyko et al., 2006] Buyko, E., Wermter, J., Poprat, M., and Hahn, U. (2006). Automatically adapting an nlp core engine to the biology domain. In Proceedings of the Joint BioLINK-BioOntologies Meeting. A Joint Meeting of the ISMB Special Interest Group on Bio-Ontologies and the BioLINK Special Interest Group on Text Data M ining in Association with ISMB, pages $65-68$.

[Casillas et al., 2016] Casillas, A., Pérez, A., Oronoz, M., Gojenola, K., and Santiso, S. (2016). Learning to extract adverse drug reaction events from electronic health records in spanish. Expert Systems with Applications, 61:235-245.

[Casillas Rubio et al., 2016] Casillas Rubio, A., Díaz de Ilarraza Sánchez, A., Gojenola Galletebeitia, K., Mendarte, L., Oronoz Anchordoqui, M., Peral, J., and Pérez Ramírez, A. (2016). Deteami research-transference project: natural language processing technologies to the aid of pharmacy and pharmacosurveillance.

[Cervantes, 2016] Cervantes, I. (2016). El español: una lengua viva. informe 2016. http://cvc.cervantes.es/lengua/espanol_lengua_viva/pdf/ espanol_lengua_viva_2016.pdf.

[Chapman et al., 2001] Chapman, W. W., Bridewell, W., Hanbury, P., Cooper, G. F., and Buchanan, B. G. (2001). A simple algorithm for identifying negated findings and diseases in discharge summaries. J Biomed Inform, 2001:34-301.

[Chapman et al., 2002] Chapman, W. W., Bridewell, W., Hanbury, P., Cooper, G. F., Buchanan, B. G., Phd, W. W. C., Bs, W. B., Bs, P. H., Phd, G. F. C. M., and Phd, B. G. B. (2002). Evaluation of negation phrases in narrative clinical reports.

[Chapman et al., 2013] Chapman, W. W., Hillert, D., Velupillai, S., Kvist, M., Skeppstedt, M., Chapman, B. E., Conway, M., Tharp, M., Mowery, D., and Deleger, L. (2013). Extending the negex lexicon for multiple languages. volume 192 of Studies in Health Technology and Informatics, pages 677-681. IOS Press. 
[Christensen et al., 2002] Christensen, L. M., Haug, P. J., and Fiszman, M. (2002). Mplus: a probabilistic medical language understanding system. In Johnson, S., editor, ACL Workshop on Natural Language Processing in the Biomedical Domain, pages 29-36. ACL.

[Coden et al., 2009] Coden, A., Savova, G. K., Sominsky, I. L., Tanenblatt, M. A., Masanz, J. J., Schuler, K., Cooper, J. W., Guan, W., and de Groen, P. C. (2009). Automatically extracting cancer disease characteristics from pathology reports into a disease knowledge representation model. Journal of Biomedical Informatics, 42(5):937-949.

[Codina et al., 2014] Codina, C. G., del Cerro, F. M., and Álvarez Cantalapiedra, A. (2014). Índice SEIS. page 8.

[Consortium et al., 1993] Consortium, O. et al. (1993). Oasis (1993-2006). URL: http://www. oasis-open. org.

[Cote et al., 1986] Cote, R., Hachinski, V., Shurvell, B., Norris, J., and Wolfson, C. (1986). The canadian neurological scale: a preliminary study in acute stroke. Stroke, 17(4):731-737.

[Cotik et al., 2016] Cotik, V., Roller, R., Xu, F., Uszkoreit, H., BuddeO, K., and SchmidtO, D. (2016). Negation detection in clinical reports written in german. BioTxtM 2016, page 115.

[da Cunha et al., 2011] da Cunha, I., Torres-Moreno, J.-M., and Sierra, G. (2011). On the development of the rst spanish treebank. In Proceedings of the 5th Linguistic Annotation Workshop, pages 1-10. Association for Computational Linguistics.

[DAB, 1999] DAB, L. (1999). About MEDLINEplus. http://www.nlm.nih.gov/ medlineplus/aboutmedlineplus.html. Last checked: 13-01-2017.

[Das and Petrov, 2011] Das, D. and Petrov, S. (2011). Unsupervised part-of-speech tagging with bilingual graph-based projections. In Proceedings of the 49th Annual Meeting of the Association for Computational Linguistics: Human Language Technologies - Volume 1, HLT '11, pages 600-609, Stroudsburg, PA, USA. Association for Computational Linguistics.

[de Cos and Moral-Benito, 2011] de Cos, P. H. and Moral-Benito, E. (2011). Eficiencia y regulación en el gasto sanitario en los países de la ocde. Documentos ocasionales-Banco de España, (7):5-36.

[de Documentación Médica (SEDOM), 2011] de Documentación Médica (SEDOM), S. E. (2011). Diccionario de siglas. URL http://www.sedom.es/diccionario/, (Last checked: 3004-2017).

[de la Concha et al., 2013] de la Concha, V. G., Salamanca, R. R.-P., Prados, L., Fernández, F. M., Iglesias, M., Vítores, D. F., and Rivilla, R. G. (2013). El español: Una lengua viva.

[de Medicamentos y Productos Sanitarios, 2013] de Medicamentos y Productos Sanitarios, A. E. (2013). Nomenclator. Last checked: 25-04-2017. 
[Degtyarenko et al., 2008] Degtyarenko, K., De Matos, P., Ennis, M., Hastings, J., Zbinden, M., McNaught, A., Alcántara, R., Darsow, M., Guedj, M., and Ashburner, M. (2008). Chebi: a database and ontology for chemical entities of biological interest. Nucleic acids research, 36(suppl 1):D344-D350.

[Deléger and Grouin, 2012] Deléger, L. and Grouin, C. (2012). Detecting negation of medical problems in french clinical notes. In Proceedings of the 2nd ACM SIGHIT International Health Informatics Symposium, pages 697-702. ACM.

[Demner-Fushman et al., 2009] Demner-Fushman, D., Chapman, W. W., and McDonald, C. J. (2009). What can natural language processing do for clinical decision support? Journal of biomedical informatics, 42(5):760-772.

[Diaz et al., 2017] Diaz, N. P. C., Vallejo, R. M., López, M. J. M., Vázquez, J. M., and Calderón, C. L. P. (2017). Annotating negation in spanish clinical texts. SemBEaR 2017, page 53 .

[Doan et al., 2014] Doan, S., Conway, M., Phuong, T. M., and Ohno-Machado, L. (2014). Natural language processing in biomedicine: A unified system architecture overview. arXiv preprint arXiv:1401.0569.

[Drouin, 2004] Drouin, P. (2004). Detection of domain specific terminology using corpora comparison. In LREC.

[EAGLES Computational Lexicons Working Group, 1996] EAGLES Computational Lexicons Working Group (1996). Preliminary recommendations on semantic encoding. Technical report, EAGLES. http://www.ilc.pi.cnr.it/EAGLES96/rep2/.

[Eclipse, 2009] Eclipse, I. (2009). for java developers. URL http://www.eclipse.org/.-2008.

[Elkin et al., 2005] Elkin, P. L., Brown, S. H., Bauer, B. A., Husser, C. S., Carruth, W., Bergstrom, L., and Wahner-Roedler, D. (2005). A controlled trial of automated classification of negation from clinical notes. BMC Med. Inf. andDecision Making, 5.

[Eltyeb and Salim, 2014] Eltyeb, S. and Salim, N. (2014). Chemical named entities recognition: a review on approaches and applications. Journal of cheminformatics, 6(1):17.

[Feng et al., 2009] Feng, S., Xiong, Y., Yao, C., Zheng, L., and Liu, W. (2009). Acronym extraction and disambiguation in large-scale organizational web pages. In Proceedings of the 18th ACM conference on Information and knowledge management, pages 1693-1696. ACM.

[Ferrucci and Lally, 2004] Ferrucci, D. and Lally, A. (2004). Uima: an architectural approach to unstructured information processing in the corporate research environment. Natural Language Engineering, 10(3-4):327-348.

[Fette et al., 2012] Fette, G., Ertl, M., Wörner, A., Klügl, P., Störk, S., and Puppe, F. (2012). Information extraction from unstructured electronic health records and integration into a data 
warehouse. In Goltz, U., Magnor, M. A., Appelrath, H.-J., Matthies, H. K., Balke, W.-T., and Wolf, L. C., editors, GI-Jahrestagung, volume 208 of LNI, pages 1237-1251. GI.

[Fiszman et al., 1998] Fiszman, M., Haug, P., and Frederick, P. (1998). Automatic extraction of pioped interpretations from ventilation/perfusion lung scan reports. Proceedings of the AMIA Symposium, pages 860-4.

[Forrey et al., 1996] Forrey, A. W., Mcdonald, C. J., DeMoor, G., Huff, S. M., Leavelle, D., Leland, D., Fiers, T., Charles, L., Griffin, B., Stalling, F., et al. (1996). Logical observation identifier names and codes (loinc) database: a public use set of codes and names for electronic reporting of clinical laboratory test results. Clinical Chemistry, 42(1):81-90.

[Foundation, 2001] Foundation, W. (2001). Wikipedia. URL http://www.wikipedia.org/, (Last checked: 30-04-2017).

[Francis and Kucera, 1979] Francis, W. N. and Kucera, H. (1964, 1971, 1979). A standard corpus of present-day edited american english, for use with digital computers (brown).

[Friedman, 1997] Friedman, C. (1997). Towards a comprehensive medical language processing system: methods and issues. In Proceedings of the AMIA annual fall symposium, page 595. American Medical Informatics Association.

[Friedman, 2000] Friedman, C. (2000). A broad-coverage natural language processing system. In Proceedings of the AMIA Symposium, page 270. American Medical Informatics Association.

[Friedman et al., 1994] Friedman, C., Alderson, P. O., Austin, J. H., Cimino, J. J., and Johnson, S. B. (1994). A general natural-language text processor for clinical radiology. Journal of the American Medical Informatics Association, 1(2):161-174.

[Friedman and Hripcsak, 1999] Friedman, C. and Hripcsak, G. (1999). Natural language processing and its future in medicine. Academic Medicine, 74(8):890-5.

[Friedman et al., 1995] Friedman, C., Hripcsak, G., DuMouchel, W., Johnson, S., and Clayton, P. (1995). Natural language processing in an operational clinical information system. Natural Language Engineering, 1(01):83-108.

[Friedman et al., 1999] Friedman, C., Knirsch, C., Shagina, L., and Hripcsak, G. (1999). Automating a severity score guideline for community-acquired pneumonia employing medical language processing of discharge summaries. In Proceedings of the AMIA Symposium, page 256. American Medical Informatics Association.

[Friedman et al., 2001] Friedman, C., Liu, H., Shagina, L., Johnson, S., and Hripcsak, G. (2001). Evaluating the umls as a source of lexical knowledge for medical language processing. In Proceedings of the AMIA Symposium, page 189. American Medical Informatics Association. 
[Gazzo et al., 2016] Gazzo, A. M., Daneels, D., Cilia, E., Bonduelle, M., Abramowicz, M., Van Dooren, S., Smits, G., and Lenaerts, T. (2016). Dida: A curated and annotated digenic diseases database. Nucleic acids research, 44(D1):D900-D907.

[Gomes and Wachsman, 2013] Gomes, J. and Wachsman, A. M. (2013). Types of strokes. In Handbook of Clinical Nutrition and Stroke, pages 15-31. Springer.

[Google, 2016] Google (2016). Google assistant. https://assistant.google.com/.

[Gurulingappa et al., 2012] Gurulingappa, H., Mateen-Rajpu, A., and Toldo, L. (2012). Extraction of potential adverse drug events from medical case reports. Journal of biomedical semantics, 3(1):15.

[Haerian et al., 2012a] Haerian, K., Salmasian, H., and Friedman, C. (2012a). Methods for identifying suicide or suicidal ideation in ehrs. In AMIA Annual Symposium Proceedings, volume 2012, page 1244. American Medical Informatics Association.

[Haerian et al., 2012b] Haerian, K., Varn, D., Vaidya, S., Ena, L., Chase, H., and Friedman, C. (2012b). Detection of pharmacovigilance-related adverse events using electronic health records and automated methods. Clinical Pharmacology \& Therapeutics, 92(2):228-234.

[Hage, 2011] Hage, V. (2011). The nih stroke scale: a window into neurological status. NurseCom Nursing Spectrum (Greater Chicago), 24:44-49.

[Hall et al., 2009] Hall, M., Frank, E., Holmes, G., Pfahringer, B., Reutemann, P., and Witten, I. H. (2009). The weka data mining software: an update. ACM SIGKDD explorations newsletter, 11(1):10-18.

[Hamosh et al., 2002] Hamosh, A., Scott, A. F., Amberger, J., Bocchini, C., Valle, D., and McKusick, V. A. (2002). Online mendelian inheritance in man (omim), a knowledgebase of human genes and genetic disorders. Nucleic acids research, 30(1):52-55.

[Harkema et al., 2009] Harkema, H., Dowling, J. N., Thornblade, T., and Chapman, W. W. (2009). Context: An algorithm for determining negation, experiencer, and temporal status from clinical reports. Journal of Biomedical Informatics, 42(5):839-851.

[Henry et al., 2016] Henry, J., Pylypchuk, Y., Searcy, T., and Patel, V. (2016). Adoption of electronic health record systems among us non-federal acute care hospitals: 20082015. Retrieved from http://dashboard.healthit.gov/evaluations/data-briefs/non-federalacute-care-hospital-ehr-adoption-2008-2015.php.

[Hettne et al., 2009] Hettne, K. M., Stierum, R. H., Schuemie, M. J., Hendriksen, P. J., Schijvenaars, B. J., Van Mulligen, E. M., Kleinjans, J., and Kors, J. A. (2009). A dictionary to identify small molecules and drugs in free text. Bioinformatics, 25(22):2983-2991.

[Hohnloser et al., 1996] Hohnloser, J. H., Holzer, M., Fischer, M. R., Ingenerf, J., and GüntherSutherland, A. (1996). Natural language processing and automatic snomed-encoding of free text: An analysis of free text data from a routine electronic patient record application with a 
parsing tool using the german snomed ii. In Proceedings of the AMIA Annual Fall Symposium, page 856. American Medical Informatics Association.

[Huang and Lowe, 2007] Huang, Y. and Lowe, H. J. (2007). A novel hybrid approach to automated negation detection in clinical radiology reports. Journal of the American Medical Informatics Association, 14(3):304-311.

[Humphreys and Lindberg, 1993] Humphreys, B. L. and Lindberg, D. (1993). The umls project: making the conceptual connection between users and the information they need. Bulletin of the Medical Library Association, 81(2):170.

[Hundt et al., 1999] Hundt, M., Sand, A., and Skandera, P. (1999). Manual of Information to Accompany the Freiburg-Brown Corpus of American English ('Frown'). Albert-LudwigsUniversität Freiburg.

[i2b2, 2006] i2b2 (2006). Health information text extraction. https://www.i2b2.org/ software/projects/hitex/hitex_manual.html.

[IBM, 2011] IBM (2011). IBM Watson. http: / / www.ibm. com/smarterplanet/us / en/ibmwatson/. Last checked: 30-09-2016.

[IBM, 2014] IBM (2014). IBM Watson Health. http://www.ibm.com/ smarterplanet/us/en/ibmwatson/health/. Last checked: 30-09-2016.

[IDC, 2014] IDC (2014). The digital universe: Driving data growth in healthcare. https://www.emc.com/analyst-report/ digital-universe-healthcare-vertical-report-ar.pdf. Last checked: 06-04-2017.

[Ide, 1998] Ide, N. (1998). Corpus encoding standard: Sgml guidelines for encoding linguistic corpora. In Proceedings of the First International Language Resources and Evaluation Conference, pages 463-70. Citeseer.

[Ide et al., 2000] Ide, N., Bonhomme, P., and Romary, L. (2000). An xml-based encoding standard for linguistic corpora. In Proceedings of the Second International Conference on Language Resources and Evaluation, pages 825-830.

[Iizuka et al., 2003] Iizuka, N., Oka, M., Yamada-Okabe, H., Nishida, M., Maeda, Y., Mori, N., Takao, T., Tamesa, T., Tangoku, A., Tabuchi, H., Hamada, K., Nakayama, H., Ishitsuka, H., Miyamoto, T., Hirabayashi, A., Uchimura, S., and Hamamoto, Y. (2003). Oligonucleotide microarray for prediction of early intrahepatic recurrence of hepatocellular carcinoma after curative resection. The Lancet, 361(9361):923-9.

[Inc., 1996] Inc., M. (1996). Medicinenet. URL http://www.medicinenet.com/, (Last checked: 01-05-2017).

[International, 2017] International, S. (2017). Snomed-ct. URL http://www.snomed.org/snomed-ct, (Last checked: 01-05-2017). 
[Jackson et al., 2017] Jackson, R. G., Patel, R., Jayatilleke, N., Kolliakou, A., Ball, M., Gorrell, G., Roberts, A., Dobson, R. J., and Stewart, R. (2017). Natural language processing to extract symptoms of severe mental illness from clinical text: the clinical record interactive search comprehensive data extraction (cris-code) project. BMJ open, 7(1):e012012.

[Jeong et al., 2011] Jeong, D.-H., Hwang, M.-G., and Sung, W.-K. (2011). Generating knowledge map for acronym-expansion recognition. In International Conference on $U$-and $E$ Service, Science and Technology, pages 287-293. Springer.

[Jessop et al., 2011] Jessop, D. M., Adams, S. E., Willighagen, E. L., Hawizy, L., and MurrayRust, P. (2011). Oscar4: a flexible architecture for chemical text-mining. Journal of cheminformatics, 3(1):41.

[Jiao and Wild, 2009] Jiao, D. and Wild, D. J. (2009). Extraction of cyp chemical interactions from biomedical literature using natural language processing methods. Journal of chemical information and modeling, 49(2):263-269.

[Joshi et al., 2006a] Joshi, M., Pakhomov, S. V., Pedersen, T., and Chute, C. G. (2006a). A comparative study of supervised learning as applied to acronym expansion in clinical reports. In AMIA.

[Joshi et al., 2006b] Joshi, M., Pedersen, T., Maclin, R., and Pakhomov, S. (2006b). Kernel methods for word sense disambiguation and abbreviation expansion. In University of Minnesota. Citeseer.

[Kanehisa and Goto, 2000] Kanehisa, M. and Goto, S. (2000). Kegg: kyoto encyclopedia of genes and genomes. Nucleic acids research, 28(1):27-30.

[Kayyali et al., 2013] Kayyali, B., Knott, D., and Van Kuiken, S. (2013). The big-data revolution in US health care: Accelerating value and innovation. page 8.

[Kim et al., 2003] Kim, J.-D., Ohta, T., Tateisi, Y., and Tsujii, J. (2003). Genia corpus-a semantically annotated corpus for bio-textmining. Bioinformatics, 19(suppl 1):i180-i182.

[Köhler et al., 2014] Köhler, S., Doelken, S. C., Mungall, C. J., Bauer, S., Firth, H. V., BailleulForestier, I., Black, G., Brown, D. L., Brudno, M., Campbell, J., et al. (2014). The human phenotype ontology project: linking molecular biology and disease through phenotype data. Nucleic acids research, 42(D1):D966-D974.

[Korkontzelos et al., 2015] Korkontzelos, I., Piliouras, D., Dowsey, A. W., and Ananiadou, S. (2015). Boosting drug named entity recognition using an aggregate classifier. Artificial intelligence in medicine, 65(2):145-153.

[Kundeti et al., 2016] Kundeti, S. R., Vijayananda, J., Mujjiga, S., and Kalyan, M. (2016). Clinical named entity recognition: Challenges and opportunities. In Big Data (Big Data), 2016 IEEE International Conference on, pages 1937-1945. IEEE. 
[Leaman et al., 2015] Leaman, R., Wei, C.-H., and Lu, Z. (2015). tmchem: a high performance approach for chemical named entity recognition and normalization. Journal of cheminformatics, 7(1):S3.

[Leech et al., 1986] Leech, G., Johansson, S., Garside, R., and Hofland, K. (1981-1986). The lob corpus, pos-tagged version (1981-1986).

[Levenshtein, 1966] Levenshtein, V. I. (1966). Binary codes capable of correcting deletions, insertions, and reversals. In Soviet physics doklady, volume 10, pages 707-710.

[Li et al., 2015] Li, C., Ji, L., and Yan, J. (2015). Acronym disambiguation using word embedding. In $A A A I$, pages $4178-4179$.

[Lill et al., 2012] Lill, C. M., Roehr, J. T., McQueen, M. B., Kavvoura, F. K., Bagade, S., Schjeide, B.-M. M., Schjeide, L. M., Meissner, E., Zauft, U., Allen, N. C., et al. (2012). Comprehensive research synopsis and systematic meta-analyses in parkinson's disease genetics: The pdgene database. PLoS Genet, 8(3):e1002548.

[Limsopatham et al., 2011] Limsopatham, N., Santos, R. L., Macdonald, C., and Ounis, I. (2011). Disambiguating biomedical acronyms using emim. In Proceedings of the 34th international ACM SIGIR conference on Research and development in Information Retrieval, pages 1213-1214. ACM.

[Liu et al., 2005] Liu, S., Ma, W., Moore, R., Ganesan, V., and Nelson, S. (2005). Rxnorm: prescription for electronic drug information exchange. IT professional, 7(5):17-23.

[Lowe and Barnett, 1994] Lowe, H. J. and Barnett, G. O. (1994). Understanding and using the medical subject headings (mesh) vocabulary to perform literature searches. Jama, 271(14):1103-1108.

[Mahoney, 1965] Mahoney, F. I. (1965). Functional evaluation: the barthel index. Maryland state medical journal, 14:61-65.

[Mann and Thompson, 1988] Mann, W. C. and Thompson, S. A. (1988). Rhetorical structure theory: Toward a functional theory of text organization. Text-Interdisciplinary Journal for the Study of Discourse, 8(3):243-281.

[Marcus et al., 1993] Marcus, M. P., Marcinkiewicz, M. A., and Santorini, B. (1993). Building a large annotated corpus of english: The penn treebank. Computational linguistics, 19(2):313-330.

[Marimon et al., 2014] Marimon, M., Bel, N., Fisas, B., Arias, B., Vázquez, S., Vivaldi, J., Morell, C., and Lorente, M. (2014). The iula spanish lsp treebank. In LREC, pages 782-788.

[Marimon et al., 2017] Marimon, M., Vivaldi, J., Bel, N., and Boronat, R. (2017). Annotation of negation in the iula spanish clinical record corpus. SemBEaR 2017, 5(36.41):43. 
[Martí et al., 2007a] Martí, M. A., Taulé, M., Bertran, M., and Màrquez, L. (2007a). Ancora: Multilingual and multilevel annotated corpora. Available: clic. ub. edu/corpus/webfm_send/13.

[Martí et al., 2007b] Martí, M. A., Taulé, M., Bertran, M., and Màrquez, L. (2007b). Ancora: Multilingual and multilevel annotated corpora. Available: clic. ub. edu/corpus/webfm_send/13.

[McInnes et al., 2011] McInnes, B. T., Pedersen, T., Liu, Y., Pakhomov, S. V., and Melton, G. B. (2011). Using second-order vectors in a knowledge-based method for acronym disambiguation. In Proceedings of the Fifteenth Conference on Computational Natural Language Learning, pages 145-153. Association for Computational Linguistics.

[Medrano et al., 2017] Medrano, I. H., Guijarro, J. T., Belda, C., Ureña, A., Salcedo, I., Espinosa-Anke, L., and Saggion, H. (2017). Savana: Re-using electronic health records with artificial intelligence. International Journal of Interactive Multimedia and Artificial Intelligence, (In Press).

[Melton et al., 2010] Melton, G. B., Moon, S., McInnes, B., and Pakhomov, S. (2010). Automated identification of synonyms in biomedical acronym sense inventories. In Proceedings of the NAACL HLT 2010 Second Louhi Workshop on Text and Data Mining of Health Documents, pages 46-52. Association for Computational Linguistics.

[Meystre et al., 2008] Meystre, S. M., Savova, G. K., Kipper-Schuler, K. C., and Hurdle, J. F. (2008). Extracting information from textual documents in the electronic health record: A review of recent research. IMIA Yearbook 2008: Access to Health Information, 2008(1):128144.

[Microsoft, 2014] Microsoft (2014). Cortana. https://www.microsoft.com/ windows / cortana.

[Morante and Daelemans, 2009] Morante, R. and Daelemans, W. (2009). A metalearning approach to processing the scope of negation. In Proceedings of the Thirteenth Conference on Computational Natural Language Learning, CoNLL '09, pages 21-29. Association for Computational Linguistics.

[Morante et al., 2008] Morante, R., Liekens, A., and Daelemans, W. (2008). Learning the scope of negation in biomedical texts. In Proceedings of the Conference on Empirical Methods in Natural Language Processing, EMNLP '08, pages 715-724.

[Moreno et al., 2000] Moreno, A., Grishman, R., López, S., Sánchez, F., and Sekine, S. (2000). A treebank of spanish and its application to parsing. In LREC.

[MSSSI Ministerio de Sanidad, 2013] MSSSI Ministerio de Sanidad, S. S. e. I. (2013). Informe anual del Sistema Nacional de Salud.

[MSSSI Ministerio de Sanidad, 2015] MSSSI Ministerio de Sanidad, S. S. e. I. (2015). Ministerio de Sanidad, Servicios Sociales e Igualdad. http://www.msssi.gob.es/ ciudadanos/hospitales.do?tipo=hospital. Last checked: 28-09-2016. 
[MSSSI Ministerio de Sanidad et al., 2010] MSSSI Ministerio de Sanidad, S. S. e. I., Red.es, Autónomas, C., and y Melilla), I. C. (2010). Las TIC en el Sistema Nacional de Salud: El programa Sanidad en Línea. page 4.

[Mutalik et al., 2001] Mutalik, P., Deshpande, A. M., and Nadkarni, P. M. (2001). Research paper: Use of general-purpose negation detection to augment concept indexing of medical documents: A quantitative study using the umls. JAMIA, 8(6):598-609.

[Narayanaswamy et al., 2003] Narayanaswamy, M., Ravikumar, K., Vijay-Shanker, K., and Ay-shanker, K. V. (2003). A biological named entity recognizer. In Pac Symp Biocomput, page 427.

[Nivre et al., 2015] Nivre, J., Agić, Ž., Aranzabe, M. J., Asahara, M., Atutxa, A., Ballesteros, M., Bauer, J., Bengoetxea, K., Bhat, R. A., Bosco, C., et al. (2015). Universal dependencies 1.2 .

[Nivre et al., 2016] Nivre, J., de Marneffe, M.-C., Ginter, F., Goldberg, Y., Hajic, J., Manning, C. D., McDonald, R., Petrov, S., Pyysalo, S., Silveira, N., et al. (2016). Universal dependencies v1: A multilingual treebank collection. In Proceedings of the 10th International Conference on Language Resources and Evaluation (LREC 2016), pages 1659-1666.

[OpenNLP, 2011] OpenNLP, A. (2011). Apache software foundation. URL http://opennlp.apache.org.

[Organization, 2006] Organization, W. H. (2006). International Statistical Classification of Diseases and Related Health Problems, 10th Revision. Version for 2006 (ICD-10).

[Organization et al., 2012] Organization, W. H. et al. (2012). International classification of diseases (icd).

[Organization et al., 2014] Organization, W. H. et al. (2014). The 10 leading causes of death in the world, 2000 and 2012. Fact sheet N, 310.

[Packer, 1999] Packer, A. L. (1999). Scielo - an electronic publishing model for developing countries. In Smith, J., Ardö, A., and Linde, P., editors, ELPUB. ICCC Press, Washington DC, USA.

[Pakhomov, 2002] Pakhomov, S. (2002). Semi-supervised maximum entropy based approach to acronym and abbreviation normalization in medical texts. In Proceedings of the 40th annual meeting on association for computational linguistics, pages 160-167. Association for Computational Linguistics.

[Pakhomov et al., 2005] Pakhomov, S., Pedersen, T., and Chute, C. G. (2005). Abbreviation and acronym disambiguation in clinical discourse. In AMIA Annual Symposium Proceedings, volume 2005, page 589. American Medical Informatics Association.

[Palomar et al., 2004] Palomar, M., Civit, M., Díaz, A., Moreno, L., Bisbal, E., Aranzabe, M. J., Ageno, A., Martí, M. A., and Navarro, B. (2004). 3lb: Construcción de una base de 
datos de árboles sintáctico-semánticos para el catalán, euskera y castellano. Procesamiento del Lenguaje Natural, 33.

[Petrov et al., 2011] Petrov, S., Das, D., and McDonald, R. (2011). A universal part-of-speech tagset. arXiv preprint arXiv:1104.2086.

[Pietrzyk, 1991] Pietrzyk, P. (1991). A medical text analysis system for german-syntax analysis. Methods of information in medicine, 30(4):275-283.

[Post, 2015] Post, W. (2015). How many patients should your doctor see each day. https://www.washingtonpost.com/news/to-your-health/wp/2014/ 05/22/how-many-patients-should-your-doctor-see-each-day/. Last checked: 30-09-2016.

[Pustejovsky et al., 2001] Pustejovsky, J., Castano, J., Cochran, B., Kotecki, M., Morrell, M., and Rumshisky, A. (2001). Extraction and disambiguation of acronym-meaning pairs in medline. Medinfo, 10(2001):371-375.

[Recasens and Martí, 2010] Recasens, M. and Martí, M. A. (2010). Ancora-co: Coreferentially annotated corpora for spanish and catalan. Language resources and evaluation, 44(4):315345 .

[Rindflesch et al., 2000] Rindflesch, T. C., Tanabe, L., Weinstein, J. N., and Hunter, L. (2000). Edgar: extraction of drugs, genes and relations from the biomedical literature. In Pacific Symposium on Biocomputing. Pacific Symposium on Biocomputing, page 517. NIH Public Access.

[Rocktäschel et al., 2012] Rocktäschel, T., Weidlich, M., and Leser, U. (2012). Chemspot: a hybrid system for chemical named entity recognition. Bioinformatics, 28(12):1633-1640.

[Rodríguez-González et al., 2015] Rodríguez-González, A., Martínez-Romero, M., Costumero, R., Wilkinson, M. D., and Menasalvas-Ruiz, E. (2015). Diagnostic knowledge extraction from medlineplus: An application for infectious diseases. In 9th International Conference on Practical Applications of Computational Biology and Bioinformatics, pages 79-87. Springer International Publishing.

[Rokach et al., 2008] Rokach, L., Romano, R., and Maimon, O. (2008). Negation recognition in medical narrative reports. Inf. Retr., 11(6):499-538.

[Rosenbloom et al., 2011] Rosenbloom, S. T., Denny, J. C., Xu, H., Lorenzi, N., Stead, W. W., and Johnson, K. B. (2011). Data from clinical notes: a perspective on the tension between structure and flexible documentation. Journal of the American Medical Informatics Association, 18(2):181-186.

[Ruch et al., 2003] Ruch, P., Baud, R., and Geissbühler, A. (2003). Using lexical disambiguation and named-entity recognition to improve spelling correction in the electronic patient record. Artificial intelligence in medicine, 29(1):169-184. 
[Salmasian et al., 2013] Salmasian, H., Freedberg, D. E., Abrams, J. A., and Friedman, C. (2013). An automated tool for detecting medication overuse based on the electronic health records. Pharmacoepidemiology and drug safety, 22(2):183-189.

[Santiso et al., 2017] Santiso, S., Casillas, A., Pérez, A., and Oronoz, M. (2017). Medical entity recognition and negation extraction: Assessment of negex on health records in spanish. In International Conference on Bioinformatics and Biomedical Engineering, pages 177-188. Springer.

[Santorini, 1990] Santorini, B. (1990). Part-of-speech tagging guidelines for the penn treebank project (3rd revision).

[Saurı et al., 2010] Saurı, R., Saquete, E., and Pustejovsky, J. (2010). Annotating time expressions in spanish timeml annotation guidelines. Technical report, Technical Report BM 2010-02, Barcelona Media.

[Savanamed, 2016] Savanamed (2016). Savana médica. http://www. savanamed.es. Last checked: 30-09-2016.

[Savova et al., 2010] Savova, G. K., Masanz, J. J., Ogren, P. V., Zheng, J., Sohn, S., KipperSchuler, K. C., and Chute, C. G. (2010). Mayo clinical text analysis and knowledge extraction system (ctakes): architecture, component evaluation and applications. Journal of the American Medical Informatics Association, 17(5):507-513.

[Segura Bedmar et al., 2013] Segura Bedmar, I., Martínez, P., and Herrero Zazo, M. (2013). Semeval-2013 task 9: Extraction of drug-drug interactions from biomedical texts (ddiextraction 2013). Association for Computational Linguistics.

[Segura-Bedmar et al., 2008] Segura-Bedmar, I., Martínez, P., and Segura-Bedmar, M. (2008). Drug name recognition and classification in biomedical texts: a case study outlining approaches underpinning automated systems. Drug discovery today, 13(17):816-823.

[Siemens, 2015] Siemens (2015). Selene Clinic. http://w5.siemens.com/spain/ web/es/healthcare/soluciones_IT/Pages/selene_clinic.aspx. Last checked: 30-09-2015.

[Skeppstedt, 2010] Skeppstedt, M. (2010). Negation detection in swedish clinical text. In Proceedings of the NAACL HLT 2010 Second Louhi Workshop on Text and Data Mining of Health Documents, pages 15-21. Association for Computational Linguistics.

[Skeppstedt, 2011] Skeppstedt, M. (2011). Negation detection in swedish clinical text: An adaption of negex to swedish. J. Biomedical Semantics, 2(S-3):S3.

[Skeppstedt, 2014] Skeppstedt, M. (2014). Extracting clinical findings from swedish health record text.

[Skeppstedt et al., 2011] Skeppstedt, M., Dalianis, H., and Nilsson, G. H. (2011). Retrieving disorders and findings: Results using snomed ct and negex adapted for swedish. In LOUHI 
2011 Health Document Text Mining and Information Analysis 2011 : Proceedings of LOUHI 2011 Third International Workshop on Health Document Text Mining and Information Analysis Bled, Slovenia, July 6, 2011., number 744, pages 11-17.

[Taboada et al., 2013] Taboada, M., Meizoso, M., Hernández, D. M., 0001, D. R., and Alonso, A. (2013). Combining open-source natural language processing tools to parse clinical practice guidelines. Expert Systems, 30(1):3-11.

[Tange et al., 1998] Tange, H. J., Schouten, H. C., Kester, A. D., and Hasman, A. (1998). The granularity of medical narratives and its effect on the speed and completeness of information retrieval. Journal of the American Medical Informatics Association, 5(6):571-582.

[Taulé et al., 2004] Taulé, M., Civit, M., Artigas, N., García, M., Màrquez, L., Martí, M. A., and Navarro, B. (2004). Minicors and cast3lb: Two semantically tagged spanish corpora. In LREC. European Language Resources Association.

[Taulé et al., 2008] Taulé, M., Martí, M. A., and Recasens, M. (2008). Ancora: Multilevel annotated corpora for catalan and spanish. In LREC. European Language Resources Association.

[The Apache Foundation, 2000] The Apache Foundation (2000). Lucene. Software.

[Tomasulo, 2002] Tomasulo, P. (2002). Chemidplus-super source for chemical and drug information. Medical reference services quarterly, 21(1):53-59.

[UNECE, 2015] UNECE, T. N. E. (2015). The problems with big data. http://www.theneweconomy.com/strategy/ big-data-is-not-without-its-problems. Last checked: 20-03-2017.

[Van Swieten et al., 1988] Van Swieten, J., Koudstaal, P., Visser, M., Schouten, H., and Van Gijn, J. (1988). Interobserver agreement for the assessment of handicap in stroke patients. Stroke, 19(5):604-607.

[Varma and Simon, 2006] Varma, S. and Simon, R. (2006). Bias in error estimation when using cross-validation for model selection. BMC bioinformatics, 7(1):91.

[Vogel, 2003] Vogel, D. (2003). Using generic corpora to learn domain-specific terminology. In Workshop on Link Analysis for Detecting Complex Behavior.

[Winter et al., 2010] Winter, A., Haux, R., Ammenwerth, E., Brigl, B., Hellrung, N., and Jahn, F. (2010). Health information systems. In Health Information Systems, pages 3342. Springer.

[Wipro, 2013] Wipro (2013). Big Data. http://www.blogilvy.co.za/ wp-content/uploads/2013/04/Big-Data.jpg. Last checked: 20-03-2017.

[Wishart et al., 2008] Wishart, D. S., Knox, C., Guo, A. C., Cheng, D., Shrivastava, S., Tzur, D., Gautam, B., and Hassanali, M. (2008). Drugbank: a knowledgebase for drugs, drug actions and drug targets. Nucleic acids research, 36(suppl 1):D901-D906. 
[Wishart et al., 2007] Wishart, D. S., Tzur, D., Knox, C., Eisner, R., Guo, A. C., Young, N., Cheng, D., Jewell, K., Arndt, D., Sawhney, S., et al. (2007). Hmdb: the human metabolome database. Nucleic acids research, 35(suppl 1):D521-D526.

[Wren, 2006] Wren, J. D. (2006). A scalable machine-learning approach to recognize chemical names within large text databases. BMC bioinformatics, 7(2):S3.

[Wren et al., 2002] Wren, J. D., Garner, H. R., et al. (2002). Heuristics for identification of acronym-definition patterns within text: towards an automated construction of comprehensive acronym-definition dictionaries. Methods of information in medicine, 41(5):426-434.

[Xu et al., 2012] Xu, H., Wu, Y., Elhadad, N., Stetson, P. D., and Friedman, C. (2012). A new clustering method for detecting rare senses of abbreviations in clinical notes. Journal of biomedical informatics, 45(6):1075-1083.

[Zahariev, 2004] Zahariev, M. (2004). Automatic sense disambiguation for acronyms. In Proceedings of the 27th annual international ACM SIGIR conference on Research and development in information retrieval, pages 586-587. ACM.

[Zeng et al., 2006] Zeng, Q. T., Goryachev, S., Weiss, S., Sordo, M., Murphy, S. N., and Lazarus, R. (2006). Extracting principal diagnosis, co-morbidity and smoking status for asthma research: evaluation of a natural language processing system. BMC medical informatics and decision making, 6(1):30.

[Zweigenbaum, 1994] Zweigenbaum, P. (1994). Menelas: an access system for medical records using natural language. Computer methods and programs in Biomedicine, 45(1):117-120. 